\title{
The population of X-ray supernova remnants in the Large Magellanic Cloud ${ }^{\star}$
}

\author{
P. Maggi ${ }^{1,2}$, F. Haberl ${ }^{1}$, P. J. Kavanagh ${ }^{3}$, M. Sasaki ${ }^{3}$, L. M. Bozzetto ${ }^{4}$, M. D. Filipović ${ }^{4}$, G. Vasilopoulos ${ }^{1}$, \\ W. Pietsch ${ }^{1}$, S. D. Points ${ }^{5}$, Y.-H. Chu ${ }^{6}$, J. Dickel ${ }^{7}$, M. Ehle ${ }^{8}$, R. Williams ${ }^{9}$, and J. Greiner ${ }^{1}$ \\ 1 Max-Planck-Institut für extraterrestrische Physik, Postfach 1312, Giessenbachstr., 85741 Garching, Germany \\ e-mail: pierre.maggi@cea.fr \\ 2 Laboratoire AIM, CEA-IRFU/CNRS/Université Paris Diderot, Service d'Astrophysique, CEA Saclay, 91191 Gif-sur-Yvette, France \\ 3 Institut für Astronomie und Astrophysik Tübingen, Universität Tübingen, Sand 1, 72076 Tübingen, Germany \\ ${ }^{4}$ University of Western Sydney, Locked Bag 1797, Penrith South DC, NSW 1797, Australia \\ 5 Cerro Tololo Inter-American Observatory, National Optical Astronomy Observatory, Cassilla 603 La Serena, Chile \\ ${ }^{6}$ Institute of Astronomy and Astrophysics, Academia Sinica, No.1, Sec. 4, Roosevelt Rd, 10617 Taipei, Taiwan, Republic of China \\ 7 Physics and Astronomy Department, University of New Mexico, MSC 07-4220, Albuquerque, NM 87131, USA \\ 8 XMM-Newton Science Operations Centre, ESA/ESAC, PO Box 78, 28691 Villanueva de la Cañada, Madrid, Spain \\ 9 Columbus State University Coca-Cola Space Science Center, 701 Front Avenue, Colombus, GA 31901, USA
}

Received 10 July 2015 / Accepted 28 September 2015

\begin{abstract}
Aims. We present a comprehensive X-ray study of the population of supernova remnants (SNRs) in the Large Magellanic Cloud (LMC). Using primarily XMM-Newton observations, we conduct a systematic spectral analysis of LMC SNRs to gain new insight into their evolution and the interplay with their host galaxy.

Methods. We combined all the archival XMM-Newton observations of the LMC with those of our Very Large Programme LMC survey. We produced X-ray images and spectra of 51 SNRs, out of a list of 59 objects compiled from the literature and augmented with newly found objects. Using a careful modelling of the background, we consistently analysed all the X-ray spectra and measure temperatures, luminosities, and chemical compositions. The locations of SNRs are compared to the distributions of stars, cold gas, and warm gas in the LMC, and we investigated the connection between the SNRs and their local environment, characterised by various star formation histories. We tentatively typed all LMC SNRs, in order to constrain the ratio of core-collapse to type Ia SN rates in the LMC. We also compared the column densities derived from X-ray spectra to H I maps, thus probing the three-dimensional structure of the LMC.

Results. This work provides the first homogeneous catalogue of the X-ray spectral properties of SNRs in the LMC. It offers a complete census of LMC remnants whose X-ray emission exhibits Fe K lines (13\% of the sample), or reveals the contribution from hot supernova ejecta (39\%), which both give clues to the progenitor types. The abundances of $\mathrm{O}, \mathrm{Ne}, \mathrm{Mg}, \mathrm{Si}$, and $\mathrm{Fe}$ in the hot phase of the LMC interstellar medium are found to be between 0.2 and 0.5 times the solar values with a lower abundance ratio $[\alpha / \mathrm{Fe}]$ than in the Milky Way. The current ratio of core-collapse to type Ia SN rates in the LMC is constrained to $N_{\mathrm{CC}} / N_{\mathrm{Ia}}=1.35\left({ }_{-0.24}^{+0.11}\right)$, which is lower than in local SN surveys and galaxy clusters. Our comparison of the X-ray luminosity functions of SNRs in Local Group galaxies (LMC, SMC, M31, and M33) reveals an intriguing excess of bright objects in the LMC. Finally, we confirm that 30 Doradus and the LMC Bar are offset from the main disc of the LMC to the far and near sides, respectively.
\end{abstract}

Key words. ISM: supernova remnants - Magellanic Clouds - ISM: abundances - supernovae: general - stars: formation X-rays: ISM

\section{Introduction}

Supernova remnants (SNRs) are the imprints of stars that died in supernova (SN) explosions on the interstellar medium (ISM). SNRs return nucleosynthesis products to the ISM, enriching and mixing it with freshly produced heavy elements. A core-collapse (CC) SN is the explosion of a massive star, and it produces large quantities of $\alpha$-group elements (e.g. O, Ne, $\mathrm{Mg}, \mathrm{Si}, \mathrm{S}$ ). Thermonuclear (or type Ia) SNe mark the disruption of a carbonoxygen white dwarf (WD) that reached the Chandrasekhar limit, although recent models suggest that sub-Chandrasekhar WDs

\footnotetext{
* Based on observations obtained with XMM-Newton, an ESA science mission with instruments and contributions directly funded by ESA Member States and NASA.
}

may also explode as type Ia SNe (Sim et al. 2010; van Kerkwijk et al. 2010; Woosley \& Kasen 2011). The thermonuclear burning front in a type Ia SN incinerates most of the progenitor to Fe-group elements. Despite the essential role of type Ia $\mathrm{SNe}$ in cosmology as standard candles, leading to the discovery that the expansion of the Universe is accelerating (Riess et al. 1998; Perlmutter et al. 1999), the exact nature of the progenitor system as either a white dwarf accreting from a companion or a merger of two white dwarves is still hotly debated (see Maoz \& Mannucci 2012, for a review).

$\mathrm{SNe}$ of either type instantaneously release a tremendous amount of kinetic energy $\left(\sim 10^{51} \mathrm{erg}\right)$ in the ISM and consequently have a profound and long-lasting impact on their surrounding environment. SN ejecta are launched to velocities in 
excess of $10^{4} \mathrm{~km} \mathrm{~s}^{-1}$, producing shock waves that heat the ISM and ejecta up to X-ray emitting temperatures $\left(>10^{6} \mathrm{~K}\right)$. SNe are the main source of energy for the ISM, in the form of kinetic energy and turbulence (e.g. Mac Low \& Klessen 2004, and references therein) or in the form of cosmic rays that are accelerated at SNR shock fronts.

X-ray observations are a powerful tool for studying SNRs (see e.g. the review of Vink 2012). While some SNRs exhibit non-thermal X-ray emission, originating in synchrotronemitting electrons accelerated up to $100 \mathrm{TeV}$ (see Koyama et al. 1995; Rho et al. 2002; Bamba et al. 2005, and references therein), most X-ray emitting SNRs have thermal spectra dominated by highly ionised species of $\mathrm{C}, \mathrm{N}, \mathrm{O}, \mathrm{Ne}, \mathrm{Mg}, \mathrm{Si}$, $\mathrm{S}$, and Fe. At the typical electron temperatures of SNR shocks ( $k T \sim 0.2-5 \mathrm{keV})$, all these astrophysically abundant elements have emission lines in the range accessible to X-ray space observatories. Thus, the thermal X-ray spectrum of an SNR encrypts precious information about the temperature, ionisation state, and chemical composition of the hot plasma (Slane 2014). This, in turn, provides clues to the evolutionary state of the remnant, ambient density (of the inter- or circum-stellar medium), age, explosion energy, and the type of supernova progenitor. The distribution of these parameters, the impact of the environment on them, and their interrelations (e.g. temperature vs. size/age, luminosity vs. ambient density) are valuable information to understand the evolution of SNRs and their role in the hydrodynamical and chemical evolution of galaxies.

Furthermore, SNRs are observable for a few tens of thousands of years. Thus, even though $\mathrm{SNe}$ are rare events in a galaxy (typically one per century or less), there will be tens or hundreds of SNRs for us to access. In our own Galaxy, the Milky Way (MW), 294 SNRs are known (Green 2014). However, studies of Galactic SNRs are plagued by the large distance uncertainties towards sources in the Galactic plane. In addition, many important $\mathrm{X}$-ray lines of $\mathrm{O}, \mathrm{Ne}, \mathrm{Mg}$, and $\mathrm{Fe}$ are emitted at energies $k T<$ $2 \mathrm{keV}$ and are readily absorbed by the high column densities in front of Galactic sources.

On the other hand, the Large Magellanic Cloud (LMC), our closest neighbour galaxy, offers an ideal laboratory for such (X-ray) studies: First, the distance towards the LMC is relatively small (50 kpc, Pietrzyński et al. 2013) and very well studied (de Grijs et al. 2014). Second, the moderate inclination angle (between $25^{\circ}$ and $40^{\circ}$, e.g. van der Marel \& Kallivayalil 2014) and the small line-of-sight depth of the LMC (between $0.3 \mathrm{kpc}$ and $1.5 \mathrm{kpc}$, van der Marel et al. 2002) mean that we can assume all LMC sources to be at a very similar distance. Third, the interstellar absorption by gas in the foreground is much smaller towards the LMC $\left(N_{\mathrm{H}}<10^{21} \mathrm{~cm}^{-2}\right)$ than towards the Galactic plane $\left(N_{\mathrm{H}}>10^{22} \mathrm{~cm}^{-2}\right)$, allowing detection of photons even in the soft X-ray regime, below $1 \mathrm{keV}$. Finally, a wealth of data is available for the LMC, allowing for easier detection and multiwavelength analysis of SNRs. For all these reasons, we aim to discover and study the complete sample of SNRs in the LMC.

While several studies exist analysing the sample of LMC remnants as a whole (see references in Sect. 3.1), they focus either on surveys with a particular instrument (at a particular wavelength, e.g. infrared or ultraviolet), or on some specific aspects (e.g. the size distribution of SNRs). In X-rays, Long et al. (1981) used the Einstein survey of the LMC to detect 26 SNRs. Later, Williams et al. (1999a) compiled a list of 37 SNRs, amongst which they studied the X-ray morphology of 31 objects with ROSAT. Since 2000, more than twenty new remnants were discovered or confirmed, primarily through XMM-Newton observations. However, the X-ray spectral analyses of LMC SNRs were presented in a wide collection of individual papers with little consistency in the instruments, spectral models, and analysis methods used. Furthermore, several known SNRs were observed for the first time with modern X-ray instrumentation during our XMM-Newton LMC survey (see Sect. 2.1) and their spectral properties are as yet unpublished (see Sect. 5.1 and Appendix D). Because of these limitations, it is not feasible to study the spectral properties of the whole population of LMC remnants with a mere survey of the available literature.

The main ambition of this work is to alleviate these limitations and provide for the first time an up-to-date study of the X-ray emission of LMC SNRs, using primarily XMM-Newton observations. To that end, we performed a systematic and homogeneous X-ray spectral analysis of all LMC SNRs for which XMM-Newton data are available. This allows meaningful comparisons of remnants at various evolutionary stages, and provides a complete census of various spectral features, such as Fe K or $\mathrm{SN}$ ejecta emission. In turn, SNRs are used as probes of their surroundings, thanks to which one can derive the chemical abundances in the hot phase of the LMC ISM, and compare those to abundances measured in older populations (globular clusters and red giant stars).

In addition, we take advantage of the availability of star formation history (SFH) maps of the LMC, based on spatially resolved stellar photometry, to investigate the connection between LMC SNRs and their local environment, characterised by different SFHs. Doing so, we devise a method to tentatively type all LMC SNRs, which can then be used to retrieve the ratio of corecollapse to type Ia SN rates in the LMC. Then, via their X-ray luminosity function, we compare SNR populations in galaxies of the Local Group (M31, M33, LMC, SMC), which have different metallicities and SFHs. Finally, we study the spatial distribution of SNRs in the LMC with respect to cool gas, star-forming regions, and stars.

This work is organised as follows: We start in Sect. 2 by presenting the X-ray observations and their processing, along with supplementary data. In Sect. 3, we compile a complete, clean sample of LMC SNRs which is used throughout the rest of the paper. The details of our data analysis methods are given in Sect. 4. The following Sections present the results of the systematic spectral analysis of LMC SNRs (Sect. 5), the SNR typing and measurement of the ratio of core-collapse to type Ia SN rates (Sect.6), the comparative study of the X-ray luminosity functions of Local Group SNRs (Sect. 7), and the spatial distribution of SNRs in the LMC (Sect. 8). Finally, we summarise our findings and offer our conclusions in Sect. 9.

\section{Observations and data reduction}

\subsection{XMM-Newton observations of the LMC}

The XMM-Newton space observatory (Jansen et al. 2001; Lumb et al. 2012) was placed in a $48 \mathrm{~h}$ highly eccentric orbit by an Ariane-V on 1999 December 10. It carries three identical X-ray telescopes, each consisting of 58 gold-coated nested Wolter-I mirrors with a focal length of $7.5 \mathrm{~m}$. Three CCD imaging cameras are placed at the focal points of each telescope. Two of them have Metal Oxide Semi-conductor (MOS) CCD arrays (Turner et al. 2001) and the third uses pnCCDs (Strüder et al. 2001). Together, they form the European Photon Imaging Camera (EPIC). Other instruments are the two Reflection Grating Spectrometers (RGS, den Herder et al. 2001) for high-resolution spectroscopy of bright on-axis point sources, and the optical monitor (OM, Mason et al. 2001), a $30 \mathrm{~cm}$ 


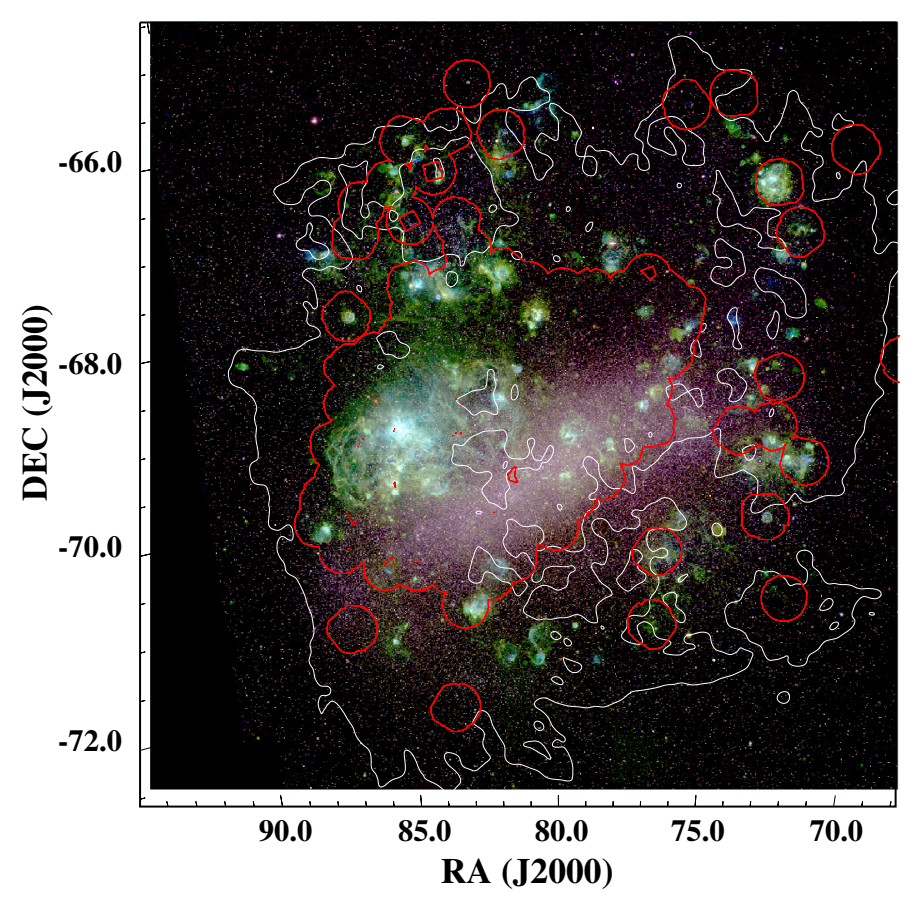

Fig. 1. The LMC in the light of [S II] (red), $\mathrm{H} \alpha$ (green), and [O III] (blue), all data from MCELS (see Sect. 2.3). The red contours delineates the coverage of the LMC with XMM-Newton, combining archival data and observations of our large survey (see Sect. 2.1). The white contours outline a LMC H I column density of $1 \times 10^{21} \mathrm{~cm}^{-2}$ (data from Kim et al. 2003).

Ritchey-Chrétien telescope observing simultaneously the central field of view in optical and ultraviolet light. However, data from RGS and OM were not used in this work.

About 200 XMM-Newton observations of the LMC were performed since the "first light" image of the observatory, of the 30 Doradus region (Dennerl et al. 2001). In most cases, one specific object is placed at the focus of the telescopes (regular or "target of opportunity" observations). Some fields were observed several times, yielding very deep exposures. For instance, the SNR N132D is used as a calibration source and regularly observed; also SN 1987A is frequently monitored (Heng et al. 2008; Maggi et al. 2012b). In these two regions, the combined exposure reaches $10^{6} \mathrm{~s}$. We have also carried out dedicated XMM-Newton observations of SNR candidates found in ROSAT, radio, and optical data (Grondin et al. 2012; Bozzetto et al. 2014; Kavanagh et al. 2015b).

Other programmes are raster surveys: the most ambitious such project was the survey of the LMC, proposed as a Very Large Programme (VLP) for XMM-Newton (PI: Frank Haberl). The survey comprises 70 pointings chosen to fill the gaps between all existing observations. This provides a contiguous field in the central region of the LMC, a strategy similar to the XMM-Newton survey of the SMC (Haberl et al. 2012b; Sturm 2012; Sturm et al. 2013). The LMC coverage with XMM-Newton, including both the 70 observations of that survey and the archival data, is shown in Fig. 1 on an optical image of the galaxy. Because the LMC is closer and has a larger extent on the sky than the SMC, all the observations combined still cover less than half of the total extent of the galaxy.

\subsection{Data reduction}

The processing of all available XMM-Newton data in the LMC region, and those of the VLP survey in particular, was done with the data reduction pipeline developed in our research group over several years. This pipeline was already used for the surveys of M31 (Pietsch et al. 2005; Stiele et al. 2011) and M33 (Pietsch et al. 2004; Misanovic et al. 2006). It was then enhanced for the analysis of the SMC survey by Richard Sturm (2012). The data reduction pipeline is similar in essence to that used for the XMM-Newton Serendipitous Source Catalogue (Watson et al. 2009), with the advantage of a better spatial accuracy (thanks to astrometric boresight corrections), and dedicated source screenings and cross-identifications. It is a collection of tasks from the XMM-Newton Science Analysis Software ${ }^{1}$, organised in bash scripts, together with other tools, in particular the FITS-file manipulation tasks of the FTOOLS package ${ }^{2}$ (Blackburn 1995). We summarise below the important steps of the pipeline.

Preparing the data: to point to the Current Calibration Files (CCFs) corresponding to each observation, a CCF index file (CIF) is created with the SAS task cifbuild. Then, using the task odfingest, the ODF summary file is extended with data extracted from the instrument housekeeping datasets. The instrument mode is also determined based on the CIF.

Creating event lists: the meta-tasks epchain and emchain produce EPIC-pn and MOS event lists, respectively. Raw events are first extracted from each exposure and CCD chip. Bad pixels are flagged. In the case of EPIC-pn, the task epreject corrects shifts in the energy scale of some pixels induced by highenergy particles hitting the detector while the offset map is calculated. Raw events are then assigned pattern and detector position information. EPIC-pn events are corrected for gain variation and charge transfer inefficiency (CTI). The calibrated events are (tangentially) projected on the sky using the task attcalc and an attitude history file (AHF), which records the attitude of the spacecraft during the observation. The AHF is created by the task atthkgen that is automatically ran before the main chain (unless the AHF already exists). EPIC-pn event times are randomised within their read-out frame. Finally, event lists from all CCDs are merged in the final list by evlistcomb.

Time filtering: times that are useful for analysis are known as good time intervals (GTIs). In particular, periods of high background must be filtered out. The pipeline identifies the background-GTIs as times when the count rate in the $(7-15) \mathrm{keV}$ band is below a threshold of $8 \times 10^{-3} \mathrm{cts} \mathrm{s}^{-1} \operatorname{arcmin}^{-2}$ and $2.5 \times 10^{-3}$ cts s$^{-1}$ arcmin $^{-2}$ for EPIC-pn and EPIC-MOS, respectively. Soft proton flares affect all detectors, so only the GTIs common to pn and MOS are used. When one instrument starts earlier or observes longer, this interval is added to the GTIs. For instance, EPIC-pn calculates an offset map before an exposure. Thus, pn exposures usually start later than those of MOS, but these times should not be vetoed, unless the background in MOS is above the threshold.

Images creation: the pipeline then produces images from the calibrated, cleaned, and background-filtered event lists. The

\footnotetext{
SAS, http://xmm.esac. esa.int/sas/

2 http://heasarc.gsfc.nasa.gov/ftools/
} 
image pixels have a size of $2^{\prime \prime} \times 2^{\prime \prime}$. All single to quadruplepixel (PATTERN $=0$ to 12 ) events with FLAG $=0$ from the MOS detectors are used. From the pn detector single and double-pixel events $($ PATTERN $=0$ to 4$)$ with $($ FLAG $\& \& \theta x f Q 000)=\theta$ (including events next to bad pixels or bad columns) are used. Below $500 \mathrm{eV}$, only single-pixel events are selected to avoid the higher detector noise contribution from the double-pixel events. Exposure maps taking into account the telescope vignetting (which is energy-dependent) are created with the task eexpmap. Images and exposure maps are extracted in various energy bands for all three cameras. Out-of-time (OoT) images are created from the EPIC-pn OoT event lists, scaled by the corresponding OoT fraction $f_{\mathrm{OoT}}{ }^{3}$, and subtracted from the source+background images. MOS and pn images are then merged, smoothed with a $10^{\prime \prime}$ full width at half maximum (FWHM) Gaussian kernel, and finally divided by the vignetted exposure maps.

Detector-background images are also created, by using XMM-Newton filter wheel closed (hereafter FWC) data, obtained with the detectors shielded from astrophysical and soft-proton backgrounds by a $1.05 \mathrm{~mm}$-thick aluminium filter. FWC data are collected several times per year, and the merged event lists of these observations are made available by the XMM-Newton Science Operations Centre ${ }^{4}$. The detector corners are always shielded from the X-ray telescopes, and the count rate in the corners is used to estimate the contribution of the instrumental background $f_{\mathrm{FWC}}$ to the science image. The FWC image is scaled by $f_{\mathrm{FWC}}$ and removed from the science image to create the background-subtracted image.

Source detection: X-ray source detection is performed simultaneously using source+background images in all available energy bands of all three instruments with the SAS meta-task edetectchain. Although this work is concerned with SNRs, i.e. extended sources, detecting point sources is highly desirable: it allows us to excise unrelated point sources from spectral extraction regions and to look for central compact objects or pulsar wind nebulae inside SNRs.

Fine-tuning for SNRs: several scripts for the analysis of the LMC SNRs were produced. For imaging purposes, all observations of an SNR are combined to produce an image centred on the source. The smoothing of the images (using the SAS task asmooth) is performed both in constant and adaptive mode. In the latter, the task calculates a library of Gaussian kernels such that the resulting images reached a minimum (Poissonian) signal-to-noise ratio of 5 everywhere. Regions of good statistics (e.g. bright sources) will be smoothed with a 10" FWHM kernel (the chosen minimum value), whereas fainter regions (diffuse emission, rims of the field of view) will be smoothed with wider kernels. The (minimum) kernel size for (adaptive) smoothing was chosen depending on the available data and brightness of the SNR under investigation. Moderately bright and faint SNRs (i.e. most of the sample) have smoothing kernel sizes of $\gtrsim 10^{\prime \prime}$ or $\gtrsim 20^{\prime \prime}$. The bright objects and SNRs in very deep fields (e.g. the field around SNR 1987A) only need shallow smoothing (kernels $\gtrsim 3^{\prime \prime}$ or $\gtrsim 6^{\prime \prime}$ ).

Images were produced in a set of energy bands tailored to the thermal spectrum of SNRs: A soft band from $0.3 \mathrm{keV}$ to $0.7 \mathrm{keV}$ includes strong lines from oxygen; a medium band from $0.7 \mathrm{keV}$

\footnotetext{
3 Values taken from the XMM-Newton Users Handbook.

4 http://xmm2.esac.esa.int/external/xmm_sw_cal/ background/filter_closed/
}

to $1.1 \mathrm{keV}$ comprises Fe L-shell lines as well as Ly $\alpha$ lines from Ne IX and Ne X; and a hard band (1.1-4.2 keV) which includes lines from $\mathrm{Mg}, \mathrm{Si}, \mathrm{S}, \mathrm{Ca}, \mathrm{Ar}$, and possibly non-thermal continuum. Thus, the composite images of SNRs provide a visual evaluation of their temperature: evolved objects with a relatively cool plasma $(0.2 \mathrm{keV} \lesssim k T \lesssim 0.4 \mathrm{keV})$ are most prominent in the soft band, those with higher temperatures $(0.4 \mathrm{keV} \lesssim k T \lesssim 1 \mathrm{keV})$ in the medium band. Only (young) SNRs with a much hotter component or a non-thermal continuum will have significant emission in the hard band as well.

\subsection{Supplementary data}

Various non-X-ray data were used to supplement the XMM-Newton observations. They allow us to assess e.g. the relation between the population of SNRs and large scale structure of the LMC (Sect. 8), or to evaluate doubtful candidates in the sample compilation (Sect. 3). Here, we present those data briefly.

Optical data: the Magellanic Clouds Emission Line Survey (MCELS, e.g. Smith et al. 2000) was carried out at the Cerro Tololo Inter-American Observatory (CTIO). It is a spatially complete, flux-limited survey with the 0.6/0.9 m Curtis Schmidt telescope of the University of Michigan. A $8^{\circ} \times 8^{\circ}$ region centred on the LMC was imaged with three narrow-band filters [S II] $] \lambda 6716,6731 \AA, \mathrm{H}^{5}$, and [O III] $\lambda 5007 \AA$. Observations with green and red broad-band filters centred at $5130 \AA$ and $6850 \AA$ were obtained to subtract stellar continua. The pixel size of the mosaicked data is $2^{\prime \prime} \times 2^{\prime \prime}$.

For optical photometry, we used results of the Magellanic Clouds Photometric Survey (MCPS, Zaritsky et al. 2004), a $U B V I$ survey of 24 million stars in the central $\sim 64 \mathrm{deg}^{2}$ of the LMC down to $V \sim 20-21 \mathrm{mag}$ (depending on crowding). Additionally, we used optical images (red continuum and $\mathrm{H} \alpha$ ) from the Southern H-Alpha Sky Survey Atlas (SHASSA Gaustad et al. 2001).

Radio: the neutral hydrogen ( $\mathrm{HI}$ ) content and structure of the LMC has been studied (at $21 \mathrm{~cm}$ ) by Staveley-Smith et al. (2003) and Kim et al. (2003). The former used data from the 64-m single-dish Parkes radio-telescope, sensitive to large-scale structures $(200 \mathrm{pc}$ to $10 \mathrm{kpc})$. They show the distribution of $\mathrm{HI}$ in a well-defined disc and three "arms" interpreted as tidal features. Several H I holes (the largest ones) are associated to supergiant shells (SGS). In Kim et al. (2003), the Parkes data are merged with data from the Australia Telescope Compact Array (ATCA) interferometer, which provides a view of the smaller structures (15 pc to $500 \mathrm{pc}$ ). The resulting map (which we used in this work) reveal the clumpiness of the H I distribution, or in their words, "the filamentary, bubbly, and flocculent structures of the ISM in the LMC". Finally, the molecular content of the LMC is assessed by the $\sim 30 \mathrm{deg}^{2}$ survey with the NANTEN telescope in the ${ }^{12} \mathrm{CO}(J=1-0)$ line (Fukui et al. 2008), from which we borrowed the velocity-integrated CO map.

Star formation history map of the LMC: the first studies of the LMC's stellar content in the 1960s suggested a different SFH than for the Milky Way (Hodge 1960, 1961). Most of the early studies used age-dating of LMC clusters. The most striking

\footnotetext{
The $\mathrm{H} \alpha$ filter included the $[\mathrm{N}$ II $] \lambda \lambda 6548,6584 \AA$ doublet in its bandpass.
} 
feature they revealed was the "Age Gap", i.e. the lack of clusters between ages of $\sim 5$ Gyr and $\sim 12$ Gyr (e.g. Da Costa 1991). Studies of field star populations (e.g. with HST, Holtzman et al. 1999; Smecker-Hane et al. 2002) reveal essentially the same results, i.e. a dearth of star formation between an initial burst ( 212 Gyr) and a second episode 4-5 Gyr ago.

The first truly global analysis of the LMC's SFH was conducted by Harris \& Zaritsky (2009). They used the results from the MCPS to perform colour-magnitude diagram fitting. They obtained a reconstruction of the star formation rate (SFR, in $M_{\odot} \mathrm{yr}^{-1}$ ) in 13 time bins and four metallicity bins, for 1380 cells, most of them having a size of $12^{\prime} \times 12^{\prime}$. Although poorly sensitive to old ages because the survey does not reach the main-sequence turn-off (MSTO) in the crowded fields ${ }^{6}$, the SFH obtained is extremely useful to study the recent and intermediate-age star formation episodes, and to compare the integrated SFH of small- and medium-scale regions. We used the SFH map to compare the local stellar populations around LMC SNRs in Sect. 6.

\section{Compiling a complete sample of LMC SNRs}

Obtaining a complete and clean census of LMC remnants is a complex task, for several reasons:

- Classification: different authors may use different criteria to classify an object as a definite SNR.

- Literature size: with the exception of the early studies, the discovery of most new objects was reported in separate papers, building up a vast literature.

- Nomenclature: an additional problem related to the previous point is the inconsistencies in the naming convention for LMC SNRs. The common names of many remnants used in the literature, especially those discovered first, are an unruly collection of various surveys and catalogues in specific wavelengths. Some are referred to after the H II complex within which they are located (e.g. "SNR in N44"), or worse, a nearby H II region (e.g. DEM L109, though it is most likely unrelated to the remnant). Other names use B1950 coordinates, with little to no consistency in the coordinates convention. Consequently, some objects were mistakenly listed twice in SNR compilations (Sect. 3.2).

To bypass these shortcomings, we performed a complete literature survey to build a list of LMC SNRs, combining all papers that either i) report the discovery or classification of one or more SNRs; ii) give a list of LMC SNRs; or iii) present new candidates (Sect. 3.1). The list is then cleaned from the wrongly identified or misclassified objects (Sect. 3.2). Unconfirmed candidates, particularly in light of new X-ray observations, are also removed. For the naming of all SNRs in the Magellanic Clouds, we made use of the acronym "MCSNR", which was pre-registered to the International Astronomical Union by R. Williams et al., who maintain the Magellanic Cloud Supernova Remnants online database ${ }^{7}$. This ensures a consistent and general naming system. Therefore, all SNRs are assigned the identifier "MCSNR JHHMM+DDMM", although we also retained the old "common names" from the literature for easy cross-identifications.

\footnotetext{
6 In the Bar the old ( $\gtrsim 4 \mathrm{Gyr}) \mathrm{SFH}$ is constrained to match that obtained with HST.

7 MCSNR, http://www. mcsnr.org/Default.aspx
}

\subsection{Literature survey}

The first extragalactic supernova remnants were found in the LMC in the 1960s. Combining Parkes observations with $\mathrm{H} \alpha$ photographs, Mathewson et al. (1963) first identified N49 as an SNR, to which Westerlund \& Mathewson (1966) soon added N63A and N132D. Less than ten years later, Mathewson \& Clarke (1973), using the same method, had already discovered 12 new SNRs ${ }^{8}$. The survey with Einstein allowed Long et al. (1981) to list 26 SNRs detected in X-rays, confirming many previously suggested candidates (based on optical or radio data). Mathewson et al. (1983) provided a catalogue of 25 SNRs with radio, optical, and X-ray results. With more observations, Mills et al. (1984) and Mathewson et al. (1984, 1985) increased the size of the sample to 32 .

In the 1990s, several new SNRs were discovered with ROSAT pointed observations (Chu et al. 1993, 2000; Smith et al. 1994), sometimes aided by optical spectroscopy (Chu et al. 1995, 1997). Since then, about twenty new remnants were discovered or confirmed in a collection of papers. Some discoveries stemmed from new radio observations (e.g. Bozzetto et al. 2012a,b; de Horta et al. 2012). The majority, though, used XMM-Newton observations, either optically selected candidates (Klimek et al. 2010), ROSAT-selected candidates (Grondin et al. 2012; Bozzetto et al. 2014, Kavanagh et al., in prep.), or serendipitously observed during the LMC VLP survey (Maggi et al. 2012a, 2014) or other programmes (Warth et al. 2014).

Several groups compiled lists of SNRs in the (Large) Magellanic Cloud(s), the purpose being to analyse some of their global properties. Filipovic et al. (1998) used Parkes surveys to study the radio spectral index and luminosityy distribution of 34 confirmed and 24 probable LMC SNRs. Williams et al. (1999a) were the first to study the X-ray morphology of all known LMC SNRs at that time. They showed ROSAT images for 31 out of their list of 37 SNRs. Blair et al. (2006, hereafter BGS06) compiled a sample of 39 SNRs in the LMC which was observed with the Far Ultraviolet Spectroscopic Explorer (FUSE) satellite. The goal was to study UV emission from SNRs, in particular in the light of highly ionised oxygen (O VI $\lambda 1032$ ). A sample of 52 confirmed and 20 candidate radio-selected SNRs was observed spectroscopically in Payne et al. (2008), but the exact list was not given. Instead, they reported the results for the $25 \mathrm{ob}-$ jects which were detected. Desai et al. (2010) studied the triggering of star formation by SNRs. To that end, they examined the young stellar objects and molecular clouds associated to LMC SNRs. Their census resulted in a list of 45 objects. A total of 54 SNRs was used by Badenes et al. (2010, hereafter BMD10) to study their size distribution. The difference in numbers stems from their including objects from unpublished sources (i.e. online catalogues). Seok et al. (2008, 2013) combined AKARI and Spitzer observatories to survey the infrared emission of LMC SNRs. They presented a list of 47 SNRs, warning that some sources in BMD10 still needed confirmation.

\subsection{Cleaning the sample: objects not included}

To build the final list of LMC SNRs, we combined objects from the older catalogues (Mathewson \& Clarke 1973; Long et al. 1981; Mathewson et al. 1983, 1984, 1985) with those reported in individual studies since then. We also included all sources present in the various compilations described in the previous

8 Counting the two distinct shells they identified in N135 (the remnants to be known as DEM L316A and DEM L316B) and including the two objects in the 30 Doradus region that they identified as candidates. 
Section. After removing all multiple occurrences of the same object, we "cleaned" the sample, searching for:

- Misclassification: the object is something else than an SNR, e.g. a superbubble. The X-ray properties of non-SNR extended sources that can be found in the field of the LMC were described in Maggi et al. (2014).

- Unconfirmed candidates: new data obtained since the classification as an SNR/candidate argue against this interpretation. This includes mainly candidates observed with $X M M-N e w t o n$ for the first time in our VLP survey. The absence of coincident X-ray emission strongly disfavours an SNR nature, unless radio and optical emission typical of SNRs is found.

- Misidentification: spurious source due to confusion (of the coordinates or nomenclature) in the literature.

Below, we describe the objects erroneously classified as SNRs or candidates and the evidence motivating the decision. These objects are listed in Table 1 and were not included in our final sample.

[BGS2006b] J0449-693: this object was observed in the UV by Blair et al. (2006) and in optical by Payne et al. (2008), although the latter used a different location, further to the south-east than the former. None of these studies gave conclusive evidence of an SNR nature (no UV lines detected, moderate $[\mathrm{S}$ II $] / \mathrm{H} \alpha$ ratio). Klimek et al. (2010) used MCELS and XMM-Newton to identify the true SNR in that region, that they named SNR0449-6921, now registered as [BMD2010] SNR J0449.3-6920 in Simbad. The X-ray emission originates from an optical shell clearly distinct from the position given for [BGS2006b] J0449-693. In BMD10, both sources are listed, although only [BMD2010] SNR J0449.3-6920 (SNR0449-6921) is the true source. This is an example of a misidentification due to coordinate confusion.

LHA 120-N 185: Blair et al. (2006) could not detect UV emission from this source (that they incorrectly listed as SNR 0453672). It was not included in the compilations from Desai et al. (2010) and Seok et al. (2013). Only BMD10 classified the source as an SNR. X-ray emission is detected, surrounded by the large, bright optical shell N 185. However, the nature of the source remains uncertain. Most likely, N 185 is actually a superbubble, and not the remnant of a single supernova (Zhang et al. 2014; Reyes-Iturbide et al. 2014).

SNR J051327-691119: this source is located north-westwards of SNR B0513-692 (which has the name MCSNR J0513-6912 in our list). Bojičić et al. (2007) present the optical and radio observations of this region, identifying the large $\left(4.1^{\prime} \times 3.3^{\prime}\right)$ shell of MCSNR J0513-6912. They detected a strong unresolved radio source at its north-western edge, that they classified as an unrelated $\mathrm{H}$ II region or background galaxy ( $\mathrm{GH}$ 6-2, see references in Bojičić et al. 2007).

In addition, they observed a faint optical shell seen in both MCELS [S II] and AAO/UKST deep H $\alpha$ images. Follow-up optical spectroscopy revealed distinct, higher $[\mathrm{S} \mathrm{II}] / \mathrm{H} \alpha$ ratios from this faint shell, prompting (Bojičić et al. 2007) to classify this shell as a new candidate SNR, J051327-691119. This region was covered by the XMM-Newton survey, revealing in details the X-ray emission of MCSNR J0513-6912 (Sect. 5, Appendix D \& F). On the other hand, the candidate J051327-691119 lacks

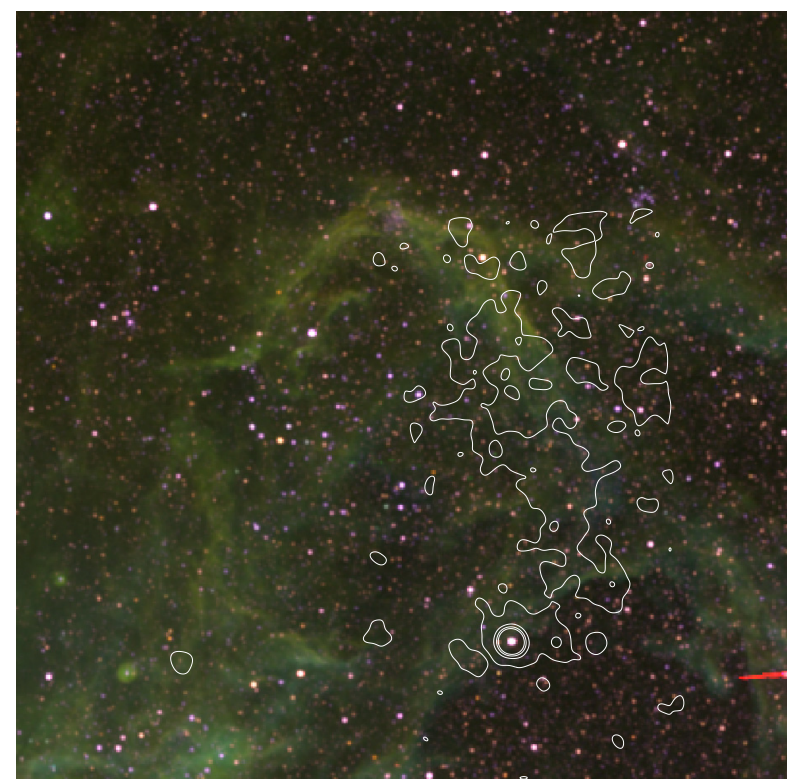

Fig. 2. The rejected SNR candidate in DEM L203 in optical lines ([S II] (red), $\mathrm{H} \alpha$ (green), and [O III] (blue), data from MCELS), with soft X-ray contours (from XMM-Newton) overlaid in white. The image spans $20^{\prime}$ across. The bright star seen in X-rays (lower right corner) is the Galactic star HD 269602.

any X-ray feature. The small extent of the source $\left(40^{\prime \prime}\right.$ diameter in $\mathrm{H} \alpha$ ) would suggest a young, X-ray bright SNR, easily detectable in observations of the XMM-Newton survey. With only weak optical evidence, a confused field in the radio, and a stringent non-detection in X-rays, one is forced to conclude that J051327-691119 is not an SNR.

LHA 120-N 204: it is only listed as an SNR in the compilation of BMD10. It was selected from the radio observations of Payne et al. (2008) where it appeared for the first time in the literature. Therefore, it was selected from radio catalogues. The "SNR" lies within the large (diameter of 14') optical shell N 204, although a size of 1' was given in Payne et al. (2008). The field 61 of the XMM-Newton survey covered this region, detecting no extended X-ray emission. With the small size of this source, bright emission is expected. Instead, an X-ray point source is detected in projection in $\mathrm{N} \mathrm{204}$, which correlates with a midIR selected AGN (MQS J052749.08-703641.7, Kozłowski et al. 2012). The background AGN is most likely the origin of the radio emission which led to the misclassification of the target as an SNR candidate.

[BMD2010] SNR J0529.1-6833: the classification as an SNR candidate (in the MCSNR online database) stems from the detection of radio emission correlating with the large optical shell DEM L203. This object is however in the compilation of "confirmed" SNRs of BMD10. Again, X-ray observations can shed light on the nature of the source. DEM L203 has no X-ray counterpart in the ROSAT catalogue. More importantly, $X M M$-Newton covered the object on three occasions during the LMC survey. Combining 35 ks of EPIC data, only unrelated large-scale diffuse emission is detected, without any correlation with the optical shell, as shown in Fig. 2. A very old age, as indicated by the large extent, might explain the lack of X-ray emission, although XMM-Newton can and did detect the largest 
P. Maggi et al.: The population of X-ray supernova remnants in the Large Magellanic Cloud

Table 1. LMC objects erroneously classified as SNRs or candidates, not included in the final sample.

\begin{tabular}{lccc}
\hline \hline \multicolumn{1}{c}{ Name } & Alternative name & Category & Ref. code \\
\hline [BGS2006b] J0449-693 & B0450-6927 & Wrong identification & BGS06 \\
LHA 120-N 185 & N185 & Wrong classification (superbubble) & PWF08 \\
SNR J051327-691119 & DEM L109 & Unconfirmed candidate & BFP07 \\
LHA 120-N 204 & B0528-7038 & Wrong identification & PWF08 \\
[BMD2010] SNR J0529.1-6833 & DEM L203 & Unconfirmed candidate & BMD10 \\
RX J0533.5-6855 & X-ray arc around & Unconfirmed candidate & LCG04 \\
30 DOR C & RX J053335-6854.9 & & \\
SNR B0538-69.3 & [BGS2006b] J0536-692 & Wrong classification (superbubble) & MFT85 \\
\hline
\end{tabular}

Notes. See text in Sect. 3.2 for a description of each object. Reference codes: MFD84: Mathewson et al. (1984); MFT85: Mathewson et al. (1985); LCG04: Lowry et al. (2004); BGS06: Blair et al. (2006); BFP07: Bojičić et al. (2007); PFW08: Payne et al. (2008); BMD10: Badenes et al. (2010).

SNRs, such as MCSNR J0450-7050 (5.7' diameter, Cajko et al. 2009) or J0506-6541 (6.8' Klimek et al. 2010). Furthermore, the MCELS image reveals no clear enhanced [S II] emission, and the source was not spectroscopically observed by Payne et al. (2008). In light of this and the absence of X-ray emission, we do not confirm the classification of this object as an SNR and did not include it in the final sample.

RX J0533.5-6855: Lowry et al. (2004) used ROSAT to study the X-ray diffuse emission around the point source RX J053335-6854.9 (referenced as RX J0533.5-6855 in Simbad) and concluded that the X-ray arc seen was a large SNR candidate; they classified the X-ray point source as a dwarf M2M3 star in the Solar neighbourhood. This region was covered in the XMM-Newton survey. The diffuse emission detected with ROSAT is found to be part of larger scale structures from the hot phase of the LMC ISM. There is no large SNR around RX J0533.5-6855.

30 DOR C: this is a large shell seen in X-rays with a nonthermal spectrum (Bamba et al. 2004; Kavanagh et al. 2015a). Its nature as a superbubble rather than a standard SNR was already recognised by Mathewson et al. (1985). It was however listed as an SNR in Blair et al. (2006, with the identifier [BGS2006b] J0536-692) and BMD10, as [BMD2010] SNR J0536.2-6912. Interestingly, there is an SNR (in projection) in 30 DOR C (MCSNR J0536-6913, Kavanagh et al. 2015a), but it was revealed only later and is most likely distinct from the non-thermal shell.

SNR B0538-69.3: the first classification as an SNR dates back to Mathewson et al. (1984), based on radio and weak optical evidence. BMD10 included that source with the wrong J2000 coordinates. Blair et al. (2006) used the correct position but did not detect UV emission from the object. B0538-69.3 is unusually bright in radio (Miroslav Filipović 2014, personal communication) considering the general lack of X-ray and optical emission. Mathewson et al. (1984) noted that the absence of X-ray emission might be due to the high $N_{\mathrm{H}}$ towards this region of the LMC. However, other SNRs are found in that region (e.g. MCSNR J0536-6913, DEM L299, the Honeycomb nebula), so a negative result with XMM-Newton is puzzling. This objects remains at best an SNR candidate.

\subsection{The final sample}

Our compilation results in a list of 59 definite SNRs. In Table C.1 we list the final sample of LMC SNRs used in this work. Basic information is given for each object: MCSNR identifier and old name, position, X-ray data available, and reference. In addition, we added columns with X-ray results: X-ray luminosity (Sect. 5 and 7), X-ray size (Sect. 4.1), and $N_{\mathrm{H}}$ fraction (Sect. 8). Finally, we give for each SNR the values of the two metrics used to assess the local stellar environment described in Sect. 6. See text in Appendix $\mathrm{C}$ for detailed description of each column.

This work focuses on the X-ray emission of LMC SNRs. Therefore, there are only confirmed SNRs in the final sample (no candidate). The resulting list provides the most complete sample of SNRs in the LMC, as far as X-rays are concerned: XMM-Newton observations exist for 51 SNRs out of the list of 59 SNRs defined here. Out of the eight objects without $X M M-N e w t o n$ data available, three were covered with Chandra, and two only by ROSAT. Only three objects have not any X-ray information available (yet), though their radio and optical properties warrant their classifications as SNR. In Sect. 7 and Sect. 9, we discuss the total number of LMC SNRs and the overall completeness of the sample.

\section{Data analysis}

\subsection{X-ray imaging}

For each SNR, we combined the smoothed images in the soft, medium, and hard bands (obtained as described in Sect. 2.2) into $\mathrm{X}$-ray composite images. These are shown in Appendix F. The same images are used to obtain X-ray contours to help in defining regions for spectral extraction (Sect. 4.2.2).

The study of the size distribution of SNRs provides clues to small-scale structures in galaxies and the energy and matter cycles in the ISM. The sample of LMC SNRs (at various levels of completeness) has been already used for such studies (e.g. Mathewson et al. 1983, BMD10). An SNR can appear to have different sizes depending on the wavelength (e.g. a larger radius in radio than in X-rays), or can have an asymmetric morphology that complicates the definition of its "size". To help future studies of the size distribution, we provide in this work the maximal extent of each SNR in X-rays, which we measured from the X-ray images and contours. The values are listed in Table C.1. The size distribution of LMC remnants, combining measurements at various wavelengths, is presented and discussed in Bozzetto et al. (in prep.). 


\subsection{X-ray spectra}

\subsubsection{Analysis method}

SNRs are extended X-ray sources, and many of those in our sample have a low surface-brightness. Consequently, the analysis of their spectra is challenging. A careful treatment of the background, both instrumental and astrophysical, is utterly important in order to obtain meaningful fits and extract the purest possible information from the source. It is not desirable to simply subtract a background spectrum extracted from a nearby region, because of the different responses and background contributions associated to different regions, and because of the resulting loss in the statistical quality of the source spectrum. An alternative method, which we used in this work, is to extract a nearby background spectrum, define a (physically motivated) model for the background and simultaneously fit the source and background spectra. Below, we explain the method in detail. Our account of the background is detailed in Appendix A. The only source for which a different method was used is SNR 1987A, as described in Sect. 5.3.

The spectral-fitting package XSPEC (Arnaud 1996) version $12.8 .0 \mathrm{~m}$ was used to perform the spectral analysis. Unless otherwise stated, spectra were rebinned with a minimum of 25 counts to allow the use of the $\chi^{2}$-statistic. Interstellar absorption was reproduced by two photoelectric absorption components (phabs and vphabs in XSPEC, where the previx " $\mathrm{v}$ " indicates that abundances can vary), one with a column density $N_{\mathrm{H} \mathrm{Gal}}$ and solar abundances for the foreground Galactic absorption, and another one with $N_{\mathrm{H} \text { LMC }}$ and LMC elemental abundances (Russell \& Dopita 1992) for absorption within the LMC. Cross-sections for photoelectric absorption were set to those of Balucinska-Church \& McCammon (1992). The foreground column density $N_{\mathrm{H} \text { Gal }}$ at the location of each analysed source is taken (and fixed) from the H I maps of Dickey \& Lockman (1990, available online on the HEASARC pages ${ }^{9}$ ).

For the analysis of one extended source, two different regions are defined: i) a source spectrum extraction region (hereafter $\mathrm{SRC}$ region); and ii) a background spectrum extraction region (hereafter BG region). Two spectra are extracted per instrument (pn, MOS1, and MOS2) from each region, one from the event list of the science observation, the second from the FWC data. The FWC spectra must be extracted at the same detector position as in the science observation, because of the strong position-dependency of the instrumental background for both pn and MOS. The SRC and BG regions are best defined in World Coordinates System (WCS, i.e. sky position ${ }^{10}$ ). Therefore, we first project the FWC data at the same sky position as the science observation, using its attitude history file and the SAS task attcalc. One can then use the same extraction regions to select FWC spectra.

The four spectra are fitted simultaneously over the $0.3 \mathrm{keV}-12 \mathrm{keV}$ range. The instrumental background model is constrained by the FWC data, and included (with tied parameters) in the spectra from the science observation. The science spectrum in the BG region therefore allows the parameters of the astrophysical X-ray background (AXB) to be determined. It is assumed that the temperature of the thermal components and the surface brightness of the thermal and non-thermal components do not vary significantly between the SRC and BG regions.

\footnotetext{
9 http://heasarc.gsfc.nasa.gov/cgi-bin/Tools/w3nh/ w3nh.pl

${ }^{10}$ This is more practical, in particular when several observations of a source with different pointings exist.
}

Thus, the appropriate temperature and normalisation parameters are linked. All background components are then accounted for, and one can explore the intrinsic emission from the source using several emission models (Sect. 4.2.3).

Regarding which instruments are used, several configurations are possible, depending on the data present. Ideally, one would use all EPIC instruments (pn+MOS1+MOS2) together. However, our analysis method requires FWC data. Those are available for all read-out modes of pn, but only for the full-frame mode of MOS, limiting the use of MOS data in some cases (e.g. MOS in Small Window mode). It also happens that the SNR is outside the MOS field of view, if it is too far off-axis or on one of the damaged chips of MOS1 (Abbey et al. 2006). In these cases only the pn spectrum is used for analysis. The contrary (only MOS spectra available) occurs in rare cases.

About $80 \%$ of the SNRs in the sample were observed only once. A few were observed twice in overlapping survey observations; the deep field centred on SNR 1987A contains four SNRs in total, and a plethora of XMM-Newton data are at hand for those. To keep the analysis the same for most sources, we restricted the number of observations analysed simultaneously to two for the latter cases. If more than two observations are available, we selected the two deepest datasets (i.e. longest flarefiltered exposure times) for analysis. Finally, N132D is a calibration target and frequently observed. It is however too bright for the full-frame mode; only Small Window and Large Window modes have been used and thus we only used the deepest pn dataset.

It was found more efficient to pre-fit the instrumental and astrophysical background of each SNR. That is, we first fitted the $(\mathrm{FWC}+\mathrm{AXB})$ EPIC-pn spectra alone and FWC MOS spectra alone. If the pre-fitting of the background components was satisfactory, their best-fit parameters were used as starting points in the final fit, which includes the SNR emission model. Doing so speeds up the process of analysing the SNR spectrum alone. It also helps, by visual examination of the background fits, to identify problematic cases, as described in Appendix A.

\subsubsection{Extraction of the spectra}

The first step of the analysis is to extract spectra for each SNR of the sample, as well as corresponding background spectra from nearby regions (using the same observation). Due to the spread in morphology and size of the SNRs, unequal properties of their background (diffuse emission and point source crowding), and their varying location on the EPIC detectors, SRC and BG regions cannot be created automatically. Therefore, extraction regions were manually defined for each SNR. For the SRC region, the constraint was simply to include all the remnant's emission and exclude unrelated point sources that might be located in projection. We used the contours taken from the X-ray image (see Sect. 4.1), combining all observations of each remnant, to identify the boundaries of the SNR emission. If the morphology of the object requires it, an arbitrary shape (polygonal region) is used instead of a circle or ellipse.

The BG regions are chosen from different locations on the pn and MOS detectors if needed, in order to be on the same CCD chip as (most of) the SNR emission. In most cases where the remnant was the target of the observation (i.e. was observed onaxis), the same BG region defined for pn can also be used for MOS data, because of the chip configuration of the latter with one central chip and six peripheral chips. Detected point sources are also excluded from the BG regions. 


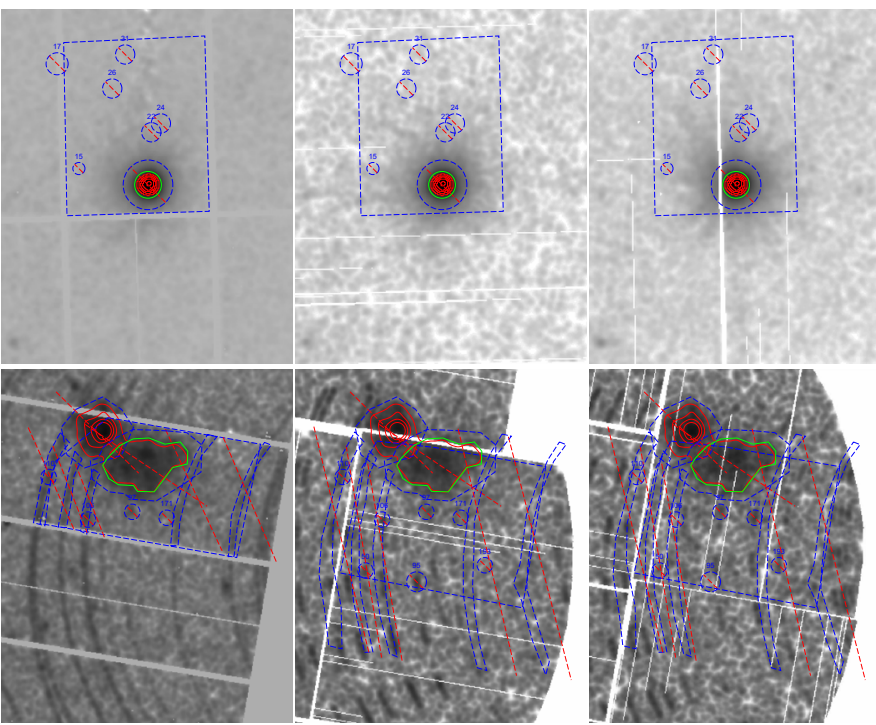

Fig. 3. Top: extraction regions used to extract spectra of MCSNR J0519-6902 from EPIC pn, MOS1, and MOS2 detectors (left to right). For the scale, we remind the reader that pn chips are $4.4^{\prime}$-wide. The $\mathrm{X}$-ray contours (in red) are used to outline the boundary of the remnant emission and set the radius of the circular SRC region (in green). The $\mathrm{BG}$ regions are shown by the blue dashed rectangle. The barred blue circles show detected point sources and a "buffer" area around the SRC region. Those are excluded from the BG region. Bottom: same for MCSNR J0547-6943, outlined by the green polygonal region. The barred blue arcs are excluded to avoid single-reflections from LMC X-1.

Two examples are shown in Fig. 3. In the simple case (that of MCSNR J0519-6902), we used a circular SRC region and the same BG regions for all EPIC detectors. In the more complex case of MCSNR J0547-6943 (or DEM L316B), we used a polygonal SRC region; the BG region is narrower for $p n$ than for MOS to fit on a single CCD chip. In addition to point sources, we excluded arc-shaped regions which are affected by instrumental artefacts (single-reflections from LMC X-1). Extraction regions for all LMC SNRs analysed in this work are shown in Appendix F.

Because of the telescope vignetting, the effective area is not constant across the extent of SNRs. To take this into account, all spectra (source and background) are extracted from vignetting-weighted event lists. These are created with the SAS task evigweight, applied on the calibrated event lists produced by our data reduction pipeline (as described in Sect. 2.2). It assigns a weight to each event of energy $E_{j}$ at detector coordinates $\left(\operatorname{det}_{j}, \operatorname{dety}_{j}\right)$, which is the inverse of the ratio of the effective area at that position to the central effective area (at the same energy):

$w_{j}=\frac{A_{0,0}\left(E_{j}\right)}{A_{\operatorname{detx}_{j}, \operatorname{dety}_{j}}\left(E_{j}\right)}$.

The corrected event lists are equivalent to that obtained with a flat instrument. For spectral analysis, a flat response file with the on-axis effective area must then be used ${ }^{11}$. Instrumental background spectra were extracted from FWC data at the same detector position as the SRC and BG regions following the method described above.

\footnotetext{
${ }^{11}$ The extraction radii for the three smallest SNRs (except SNR 1987A which is handled as a point source) result in PSF losses less than $15 \%$.
}

\subsubsection{Spectral models}

The model of the SNR emission is built iteratively, in increasing order of complexity. First, a one-component collisional ionisation equilibrium (CIE) model (vapec in XSPEC) is tried. The elemental abundances are initially set to the values measured by Russell \& Dopita (1992). Their abundance of silicon is highly uncertain, however, and therefore we use an initial value of half solar for Si. Analysis of the residuals and goodness-of-fit reveals if and how the model can be improved, by either thawing some elemental abundances, or switching to a non-equilibrium ionisation (NEI) model (vpshock in XSPEC). Abundance effects are manifest when all lines from one element are over- or underestimated compared to the continuum or other elements, while the signatures of NEI are in the residuals from different ions of the same element (e.g. relative strengths of O VII/O VIII lines). We evaluate the significance of the fit improvements (if any) with F-tests. We refer to these one-component models as "1T" (one temperature).

A second component is added if needed; those are the " $2 \mathrm{~T}$ " SNRs. Again we start with CIE, then assess whether there is need for NEI or free abundances in the second component. For several SNRs, the analysis of X-ray colour images already hints at the presence of two components, with e.g. a different temperature, $N_{\mathrm{H}}$, or abundance pattern. This iterative process is done until a satisfactory fit is achieved, at which point $90 \%$ C.L. errors are computed for all free parameters. More complicated models may be applied/needed for a handful of SNRs, particularly amongst the brightest ones. These cases are presented in Sects. 5.2 and 5.3

\section{The X-ray spectral properties of LMC SNRs}

\subsection{General properties}

Out of the sample of 59 SNRs and 51 with XMM-Newton data available, 45 are fitted with $1 \mathrm{~T}$ or $2 \mathrm{~T}$ models, while 6 , amongst the brightest, are fitted with more complex models (see Sects. 5.2 and 5.3). Six previously known SNRs have been covered by our VLP survey, giving the opportunity to study their spectra with XMM-Newton for the first time. Among these, MCSNR J0534-6955 and J0547-7025 were observed with Chandra (Hendrick et al. 2003). Reyes-Iturbide et al. (2008) analysed only MOS data of MCSNR J0518-6939 from an archival observation, while we now have EPIC-pn data. We show the XMM-Newton spectra from these SNRs in Appendix D.

The results of the spectral analysis for the $1 \mathrm{~T} / 2 \mathrm{~T}$ sample are given in Appendix E (Table E.1). All relevant parameters are listed with their $90 \%$ C.L. uncertainties: the fitted LMC absorption column density (Col. 2), plasma temperature $k T$ (3), ionisation age $\tau$ (4), emission measure EM (5), and abundances (6). When a second component is used, its parameters are given in Cols. (7)-(11). The first component is the one with higher EM. The quality of the fits are evaluated by the $\chi^{2} / v$ of Col. (12), where $v$ is the number of degrees of freedom. The reduced $\chi^{2}$ values $\left(\chi_{\text {red }}^{2}\right)$ are also listed in Col. (12). The median $\chi_{\text {red }}^{2}$ is 1.16 . $90 \%$ of the fitted objects have a reduced $\chi^{2}$ less than 1.4 .

In 32 cases, the SNR is fitted with, or the available data only require, a one component model. Amongst these, 9 do not show significant NEI effects and are fitted with a CIE model. Using a NEI model for these did not result in a statistically significant improvement, neither in the goodness-of-fit sense, nor in terms of residuals. Moreover, the ionisation ages $\tau$ in these cases are high and poorly constrained. Therefore we list the CEI model 
parameters. In the 23 remaining " $1 T$ " objects, better fits are obtained with an NEI model. The plasma temperature for the "1T SNRs" clusters in the $0.25 \mathrm{keV}-0.45 \mathrm{keV}$ range. The highest values of temperature (above $1 \mathrm{keV}$ ) are associated with the smallest ionisation ages. In at least some cases, this could be an artefact of the analysis due to insufficient data. The ionisation age $\tau$ of this sample is broadly distributed around a median value of $1.7 \times 10^{11} \mathrm{~s} \mathrm{~cm}^{-3}$.

There are 13 SNRs in the $2 \mathrm{~T}$ sample. Two objects are fitted with two CIE component models (MCSNR J0530-7008 and J0517-6759). The rest was fitted with two NEI components, although for three SNRs the ionisation age of one of the components was unconstrained and on the high end $\left(\tau \gtrsim 10^{13} \mathrm{~s} \mathrm{~cm}^{-3}\right)$, indicating a plasma close to or in CIE. The median $\tau$ of the main component (i.e. that with higher emission measure) for the $2 \mathrm{~T}$ sample is slightly higher $\left(5-7 \times 10^{11} \mathrm{~s} \mathrm{~cm}^{-3}\right)$ than that of the $1 \mathrm{~T}$ sample, but low number statistics preclude a direct comparison. The temperature distribution is bimodal: one component has a median temperature of $k T=0.31 \mathrm{keV}$, the second a higher median of $0.8 \mathrm{keV}$. In several cases the high- $k T$ component also requires a different abundance pattern, revealing SN ejecta (Sect. 5.5).

For nine SNRs, the data did not require or allow elemental abundances to be fitted. For a few cases, this happens because the spectrum is contaminated by a bright pulsar (N157B and MCSNR J0540-6920), or by LMC X-1 (MCSNR J0540-6944), and the thermal emission is not well separated by XMM-Newton. The other SNRs fitted with abundances from Russell \& Dopita (1992, RD92 in Table E.1) are relatively faint. The limited available data therefore prevent the use of free abundances in the fits.

Oxygen and iron are the main contributors to the $0.5 \mathrm{keV}-2 \mathrm{keV} \mathrm{X}$-ray emission for the relevant plasma temperatures. Consequently, they are the first elements for which abundances can be fitted. Out of the 45 1T/2T SNRs, 35 have at least free $\mathrm{O}$ and $\mathrm{Fe}$ abundances. Neon and magnesium also have prominent lines routinely detected below $2 \mathrm{keV}$, and their abundances were fitted in 33 and 30 SNRs, respectively. Silicon is detected and its abundance fitted, in 23 SNRs. This subset has a higher median temperature $(k T \sim 0.6 \mathrm{keV})$ than the whole sample, as expected. Indeed, $\mathrm{Si}$ emission becomes prominent for higher temperatures than, say, $\mathrm{O}, \mathrm{Ne}$, or Fe. While obvious and fitted in all the brightest SNRs, which are younger/hotter, lines of sulphur are not detected in most 1T/2T SNRs. Only a handful (MCSNR J0534-6955, J0547-7025, N63A) allow to fit the $\mathrm{S}$ abundances. All have plasma temperatures in excess of $0.8 \mathrm{keV}$. The fitted abundance patterns can be used to type the supernova progenitor, if ejecta are detected (Sect. 5.5), or to measure metallicity of the LMC ISM (Sect. 5.6).

\subsection{The analysis of the brightest SNRs}

For six of the brightest SNRs, the simple 1T/2T models approach was clearly insufficient to satisfactorily model the spectra. This is expected, because on the one hand, the exquisite statistical quality of these spectra imply that even a two-component model is not adequate to reproduce the complex multi-phase structure in these objects. On the other hand, the very young SNRs, in addition to a small ambient medium contribution, are dominated by ejecta. Because of stratification of the ejecta heated by the reverse shock, elements synthesised at different radii in the SN explosion can have distinct spectral properties.

All the "bright SNR" sample was observed in individual XMM-Newton and Chandra pointings. Detailed results are published in several papers (references are given below), with which our results were never at odds. Here, we used multitemperature empirical models to reproduce the spatially integrated spectra. Thus, we can i) derive accurate X-ray fluxes, so that the luminosity function (Sect.7) is complete at the bright end, ii) measure the properties of the Fe $\mathrm{K}$ emission, if present (see Sect. 5.4), and iii) obtain spectral properties (e.g. $N_{\mathrm{H}}, k T$, $\tau$ ) for statistical studies and comparison of their distributions for various sub-samples (Sect. 7). The adopted models are described below. The spectral parameters are given in Table E.2 and Table E.3.

DEM L71 (MCSNR J0505-6753): DEM L71 is a notorious type Ia SNR, owing to the detection of iron-rich ejecta (e.g. Hughes et al. 1995). van der Heyden et al. (2003) presented the XMM-Newton EPIC and RGS results for this remnant, and Hughes et al. (2003) those obtained with Chandra observations. Different conditions are measured in the shell and central regions. It is then unsurprising that a $2 \mathrm{~T}$ model as used for other SNRs did not produce acceptable fits. Instead, we obtained satisfactory results with three components: Two components ("Felow $k T$ " and "Fe-high $k T$ ") had $\mathrm{Si}, \mathrm{S}$, and Fe (the main nucleosynthesis products of Ia $\mathrm{SNe}$ ) freed and common to the two components, while other metals were set to zero. These components account for the ejecta-rich emission, as well as the $\mathrm{Si}, \mathrm{S}$, and $\mathrm{Fe}$ contribution of the ISM. A third component, with $\mathrm{O}, \mathrm{Ne}$, and $\mathrm{Mg}$ abundances free and $\mathrm{Si}, \mathrm{S}, \mathrm{Fe}$ set to zero, accounts for the bulk of ISM emission.

In addition, $\mathrm{Fe} \mathrm{K}$ emission is clearly detected, pointing to the presence of very hot ejecta $(k T>2 \mathrm{keV})$. The statistical weight of this feature remains small. Therefore, instead of adding another thermal component, we modelled the line with a Gaussian. The parameters of the Fe $\mathrm{K}$ line are used in comparison with other SNRs in Sect. 5.4. The ejecta components have best-fit temperatures of $\sim 0.4 \mathrm{keV}$ and $\sim 0.9 \mathrm{keV}$ (Table E.2). The ionisation age of the cooler component is twice that of the hotter one. The ISM component has a temperature of $k T=0.46 \mathrm{keV}$, the same as measured with Chandra (Hughes et al. 2003), and in between the two temperatures used for the shell emission by van der Heyden et al. (2003).

N103B (MCSNR J0509-6844): the spectrum of N103B is remarkable because of the numerous lines from highly ionised metals: Si XII and Si XIV, S XV and (marginally) S XVI, Ar XVII, and Ca XIX. A strong Fe K blend is also detected. We fit the spectrum with the same three-temperature model as for DEM L71. One component had abundances fixed to RD92, accounting for the ISM emission. Two components with different $k T$ and $\tau$ were used to reproduce the (dominating) ejecta emission. All relevant elements (O, Ne, Mg, Si, S, Ar, Ca, and Fe) were freed, but common to both components. A Gaussian was also included to fit the Fe $\mathrm{K}$ feature.

With this model, the spectrum of N103B is well reproduced across the whole $0.3 \mathrm{keV}-8 \mathrm{keV}$ band. The results are comparable to those of van der Heyden et al. (2002, focusing on XMM-Newton data) and Lewis et al. (2003, with Chandra), especially regarding: i) the column density $N_{\mathrm{H}} \sim 3 \times 10^{21} \mathrm{~cm}^{-2}$; ii) the presence of one high ionisation age component (at $k T \sim 0.7 \mathrm{keV})$ and a hotter $(1.6 \mathrm{keV})$ underionised component. Because the Fe $\mathrm{K}$ blend is modelled separately with a Gaussian, the fitted temperature of the hottest component is lower than in the previous references; iii) high abundances of $\mathrm{S}$, Ar, and $\mathrm{Ca}$. 
N132D (MCSNR J0525-6938): Behar et al. (2001) presented the XMM-Newton observations of N132D from the Performance Verification programme. Results of the Chandra ACIS-S observations can be found in Borkowski et al. (2007). Both instruments spatially resolve the SNR into regions with different spectral properties. Therefore, though a three-temperature model can reproduce the main features of the spectrum (thus allowing us to accurately measure the integrated flux of the remnant), strong residual structures are seen between $0.5 \mathrm{keV}$ and $1 \mathrm{keV}$, where the strongest variations are observed (lines of $\mathrm{O}, \mathrm{Ne}, \mathrm{Fe}$ ).

The best fit is obtained with a cool $(\sim 0.5 \mathrm{keV})$ component with abundances close to the normal LMC values (i.e. it represents a blast wave component) that dominates the soft emission (below $1.5 \mathrm{keV}$ ). A second component with $k T \sim 1 \mathrm{keV}$ is characterised by enriched levels of $\mathrm{O}, \mathrm{Ne}$, and $\mathrm{Mg}$, as well as a higher column density $\left(\sim 10^{22} \mathrm{~cm}^{-2}\right)$. This component thus describes the bulk of the ejecta emission, and accounts for most of the Si and S emission. Finally, the presence of highly ionised iron is evident from the $6.69 \mathrm{keV}$ line (see Table 2), corresponding to the $\mathrm{K} \alpha$ energy of Fe XXV. This indicates a third, very hot component $(\sim 5 \mathrm{keV})$. In this component only $\mathrm{Fe}, \mathrm{Ar}$, and $\mathrm{Ca}$ are included. The two latter elements improve the residuals around $3.1 \mathrm{keV}$ (Ar XVII), and 3.9/4.1 keV (Ca XIX and Ca XX). These $\mathrm{K}$ lines were already mentioned in the early XMM-Newton results (Behar et al. 2001).

0519-69.0 (MCSNR J0519-6902): the SNR was observed early in the Chandra and XMM-Newton missions. In addition, the LMC survey covered the source, at an off-axis angle of $\sim 9^{\prime}$, adding $23 \mathrm{ks}$ and $27 \mathrm{ks}$ to the existing $8 \mathrm{ks}$ and $46 \mathrm{ks}$ of fullframe pn and MOS data, respectively. Spectra from the two observations were fitted simultaneously. 0519-69.0 exhibits strong lines of $\mathrm{Si}, \mathrm{S}, \mathrm{Ar}$, and $\mathrm{Ca}$, as well as prominent $\mathrm{Fe} \mathrm{L}$ and $\mathrm{K}$ blends. To reproduce the spectra we used the multi-component approach of Kosenko et al. (2010), who extensively studied the XMM-Newton and Chandra data.

First, one NEI component with LMC abundances accounts for circumstellar medium (CSM) or ISM emission. Then, one NEI component for each (group of) element(s) having detected lines: oxygen, silicon and sulphur, argon and calcium, and iron. In the latter case two NEI components with distinct parameters are used, as the spectrum evidently includes both medium temperature and very hot iron. Due to the low count rate, and therefore statistical weight, of the Fe K blend, the hot iron component was driven to fit lower energy lines instead. To alleviate this issue we fitted the high-energy part of the spectrum separately with this component, then froze the best-fitting parameters in the global fits. Residuals around $0.72 \mathrm{keV}$ (lines of Fe XVII) were fitted with an additional Gaussian line.

0509-67.5 (MCSNR J0509-6731): XMM-Newton observed the SNR for $\approx 40 \mathrm{ks}$ in 2000 , with pn operated in Large Window mode. This dataset is presented in Kosenko et al. (2008), while Warren \& Hughes (2004) reported the spectral and imaging analysis of a Chandra observation. Finally, Badenes et al. (2008) attempted to reproduce spectra from both instruments using a grid of hydrodynamical models and an X-ray emission code. Inconsistencies between pn and MOS spectra were found, with lines in the pn spectrum (red-)shifted relative to those in MOS spectra by about $1 \%$. This is likely a gain issue of the pn instrument. We discarded spectra from the MOS instruments, as they were operated in Small Window mode, for which no FWC data are available. To get the spectral model to match the observed energies of atomic lines, we freed the "redshift" parameter available in XSPEC models, which allows an ad hoc change of the energy scale. Satisfying results were obtained for a shift of $\approx 1 \%$, which is the measured pn/MOS discrepancy Kosenko et al. (2008).

As for J0519-6902, lines from heavy elements are prominent, and we used a multi-component model. Si, S, and $\mathrm{Ar}$ were grouped in a NEI component, and shared the same temperature and ionisation age. Another NEI component modelled the continuum+lines emission from the CSM/ISM. No Si, $\mathrm{S}$, Ar, or $\mathrm{Ca}$ were included in this component. Iron was included in two NEI components, one with a medium temperature $(\sim 1.4 \mathrm{keV})$ and a high- $k T$ one $(\sim 11 \mathrm{keV})$ that reproduces the strong $\mathrm{Fe} \mathrm{K}$ line. The latter component also includes calcium. Even with this model, residuals remained around $\mathrm{Fe}$ lines $(0.72 \mathrm{keV}$ and $1.22 \mathrm{keV})$, which we fitted with two Gaussian lines. The very high temperature of the second Fe component is atypical for SNRs, but was also suggested in Chandra data by Warren \& Hughes (2004). The SNR exhibits a high-energy continuum tail, which previous studies tried to reproduce with non-thermal models. This tail can also be reproduced with the Bremsstrahlung continuum of a high- $k T$ thermal model, driving our fit to temperatures above $10 \mathrm{keV}$. Furthermore, we already noted the energy shift issue of the pn spectra, that results in a small centroid energy of the Fe $\mathrm{K}$ line for a given fitted $k T$. Given this caveats, it remains unclear whether the $11 \mathrm{keV}$ plasma is physical.

\subsection{Update on the monitoring of SNR $1987 A$}

SN 1987A, the nearest supernova in almost 400 years, was discovered in the LMC on 23 February 1987. It is exceptional in many ways and has been extensively studied ever since. We have the unique opportunity to follow the early evolution of a supernova remnant (hence the use of the identifier "SNR 1987A"12). Results from the many existing XMM-Newton observations of SNR 1987A are presented in Haberl et al. (2006), Heng et al. (2008), and Sturm et al. (2010). Maggi et al. (2012b, hereafter M12) analysed data from the 2007-2011 monitoring, focusing on the rapid evolution of the X-ray light curve and the properties and evolution of the $\mathrm{Fe} \mathrm{K}$ lines, which were detected unambiguously for the first time. However, the spectral parameters (except fluxes and Fe K line properties) were not given in M12. We take advantage of this work to give these detailed results, and include an unpublished observations (ObsID 0690510101) performed on December 2012, after M12 was released.

All spectra from SNR 1987A were extracted from a circular region centred on the source, with a radius of $25^{\prime \prime}$. The use of spatially integrated spectra is dictated by the small radius of the source (still less than 1", Helder et al. 2013), which is completely unresolved by XMM-Newton. The background spectra were extracted from a nearby point-source-free region common to all observations. Only single-pixel events (PATTERN $=0$ ) from the pn detector were selected. Contrary to all other SNRs in this work, the background spectra were not modelled but subtracted from the source spectra. We used the same threecomponent plane-parallel shock model as in M12, with one fixed-temperature component $(k T=1.15 \mathrm{keV})$ and free abundances of $\mathrm{N}, \mathrm{O}, \mathrm{Ne}, \mathrm{Mg}, \mathrm{Si}, \mathrm{S}$, and Fe. EPIC-pn spectra from all seven epochs of the monitoring are fitted simultaneously between $0.2 \mathrm{keV}$ and $10 \mathrm{keV}$, with common abundances and

\footnotetext{
${ }^{12}$ In our nomenclature SNR 1987A is also given the name MCSNR J0535-6916.
} 
Table 2. Fe K line properties of LMC SNRs.

\begin{tabular}{llccccc}
\hline \hline \multirow{2}{*}{ MCSNR } & \multirow{2}{*}{ Alt. name } & \multirow{2}{*}{ type } & \multicolumn{2}{c}{ Energy centroid (eV) } & \multicolumn{2}{c}{ Line luminosity $\left(10^{42} \mathrm{ph} \mathrm{s}^{-1}\right)$} \\
& & & XMM-Newton & Suzaku & XMM-Newton & Suzaku \\
\hline J0509-6731 & B0509-675 & Ia & $6432_{-27}^{+29}$ & $6425_{-15}^{+14}$ & $0.87 \pm 0.21$ & $0.96 \pm 0.12$ \\
J0505-6753 & DEM L71 & Ia & $6494 \pm 58$ & - & $0.26_{-0.09}^{+0.08}$ & - \\
J0509-6844 & N103B & Ia & $6514_{-32}^{+31}$ & $6545 \pm 6$ & $5.10 \pm 0.87$ & $6.43 \pm 0.30$ \\
J0519-6902 & B0519-690 & Ia & $6543_{-31}^{+28}$ & $6498_{-8}^{+6}$ & $1.71 \pm 0.45$ & $2.78 \pm 0.15$ \\
J0526-6605 & N49 & CC & - & $6628_{-26}^{+29}$ & $<4.75^{a}$ & $0.54 \pm 0.12$ \\
J0535-6916 & SNR 1987A & CC & $6635 \pm 70$ & $6646_{-54}^{+55}$ & $0.64 \pm 0.18$ & $0.57 \pm 0.24$ \\
J0535-6602 & N63A & CC & $6683_{-99}^{+88}$ & $6647_{-17}^{+16}$ & $2.36_{-1.08}^{+1.03}$ & $2.57 \pm 0.36$ \\
J0525-6938 & N132D & CC & $6685_{-14}^{+15}$ & $6656 \pm 9$ & $4.58 \pm 0.58$ & $5.47 \pm 0.51$ \\
\hline
\end{tabular}

Notes. Suzaku results are from Yamaguchi et al. (2014). ${ }^{(a)} 3 \sigma$ upper limit. ${ }^{(b)}$ The quoted numbers are average values over the last six epochs, and the uncertainties are the rms scatter. Note that we found the energy centroid to evolve rapidly at recent epochs (see Sect. 5.3).

$N_{\mathrm{H} \text { LMC. }}$ To characterise the Fe K line, we performed separate fits in the range (5-8) $\mathrm{keV}$ on the non-rebinned spectra using the C-statistic (Cash 1979). We used a Bremsstrahlung model for the continuum and a Gaussian for the Fe K line complex.

The simultaneous (broad-band) fit was satisfactory, with $\chi^{2}=5114.2$ for 4109 degrees of freedom (reduced $\chi_{r}^{2}=1.24$ ). Spectral results are the same as in M12. We give the bestfit parameters for all seven epochs in Table E.3. We list soft $(0.5 \mathrm{keV}-2 \mathrm{keV})$ and hard $(3 \mathrm{keV}-10 \mathrm{keV}) \mathrm{X}$-ray fluxes at all epochs, with $3 \sigma$ uncertainties $(99.73 \%$ confidence level, C. L.) in Table E.3. Echoing the findings of M12, we see that the soft X-ray flux keeps increasing after the 25th anniversary of SNR 1987A. Since 2011, however, the rate of increase has dropped below $10 \%$ per year, showing that subsequent observations of SNR 1987A with XMM-Newton are highly desirable to follow the evolution of the X-ray flux and to identify the turnover point.

The central energy, $\sigma$-width, total photon flux and equivalent width (EW) of the Fe K feature are also listed in Table E.3. Up to December 2011 the results are the same as in M12. The new data point (December 2012) reveals a line with roughly the same flux but a significantly higher central energy $\left(6.78_{-0.05}^{+0.06} \mathrm{keV}\right)$ than previously $(6.60 \pm 0.01 \mathrm{keV}$, averaging the earlier measurements). This hardening likely indicates an increased contribution from highly ionised iron (Fe XXVI) that prior to 2012 was either absent (as iron was in lower ionisation stages) or too weak to be detected. With the resolution of pn and the statistics in our hand, it is not possible to resolve the $\mathrm{K}$-shell lines from various $\mathrm{Fe}$ ions, which will become possible with next-generation X-ray calorimeters onboard Astro-H (Takahashi et al. 2012) or Athena (Barret et al. 2013).

\subsection{Fe K emission from LMC SNRs}

Yamaguchi et al. (2014) used Suzaku to systematically search for Fe K emission from Galactic and LMC SNRs. Fe K $\alpha$ emission was detected in 23 SNRs, including seven remnants in the LMC. Their essential finding is that the centroid energy of the $\mathrm{Fe} \mathrm{K}$ emission, determined by the ionisation state of iron, is a powerful tool for distinguishing progenitor types. Indeed, the Fe $\mathrm{K}$ emission of type Ia remnants is significantly less ionised than in CC-SNRs. Furthermore, there is a positive correlation between the $\mathrm{Fe} \mathrm{K} \alpha$ line luminosity and centroid energy within each progenitor group.
Because the Fe $\mathrm{K}$ blend is a promising typing tool, we extended the search for Fe K emission of Yamaguchi et al. (2014) to all LMC SNRs observed with XMM-Newton. Compared to the Suzaku sample, the coverage is more complete (i.e. more SNRs observed) and more sensitive (the EPIC-pn effective area is slightly higher than that of Suzaku's XIS, even combining all detectors), and Fe $\mathrm{K}$ can potentially be detected from more SNRs.

In Table 2, we give the results for all LMC SNRs with detected Fe K emission, ranked by increasing centroid energy. The $X M M-N e w t o n$ and Suzaku results are consistent within the uncertainties. Strikingly, we found Fe K emission undetected with Suzaku for only one source, DEM L71. Its line luminosity is smaller than from any other LMC remnant. Likely, this fact and the smaller effective area of XIS explain why it was undetected in the $100 \mathrm{ks}-$ long Suzaku observation of the remnant (Hiroya Yamaguchi 2014, personal communication). Furthermore, the second faintest Fe K line from LMC SNRs is found in N49. With $X M M-N e w t o n$ one does not formally detect the line. Including a Gaussian at the energy measured with Suzaku, the XMM-Newton spectrum allows a line flux an order of magnitude above that actually detected. This is only a statistical issue. Indeed, there are less than $10 \mathrm{ks}$ of EPIC-pn data available, which is no match to the $158 \mathrm{ks}$ spent by Suzaku on N49 when detecting the Fe K line.

The properties of the Fe K emission from DEM L71 fit well with its type Ia nature. Furthermore, Yamaguchi et al. (2014, their Fig. 1, right) used simple (one-dimensional) theoretical models of type Ia SNe exploding in uniform ambient media of various densities to predict the luminosity and energy of the line. Even with this simplistic approach, they are able to reproduce all the parameter space spanned by type Ia SNRs. In this context, the location of DEM L71 in the Fe K luminosity-energy diagram is well reproduced by a delayed-detonation model with a rather high explosion energy $\left(1.4 \times 10^{51} \mathrm{erg}\right.$, DDTa in Badenes et al. 2003, 2005), in an ambient medium of density $\rho=2 \times$ $10^{-24} \mathrm{~g} \mathrm{~cm}^{-3}$, at age between $2000 \mathrm{yr}$ and $5000 \mathrm{yr}$. This is in line with the measured density and age of DEM L71 (van der Heyden et al. 2003; Ghavamian et al. 2003). Furthermore, the DDTa model predicts a silicon-to-iron mass ratio of 0.08 , close to that measured in X-rays ( $\sim 0.15$, Hughes et al. 2003; van der Heyden et al. 2003). Since the hot, $\mathrm{K} \alpha$-emitting iron was previously overlooked, the $M_{\mathrm{Si}} / M_{\mathrm{Fe}}$ ratio should be even lower, closer to the prediction of the DDTa model. 
The dearth of Fe K-emitting remnants, aside from the combined XMM-Newton/Suzaku sample (eight objects), is somehow expected. Indeed, most of the SNRs have plasma temperatures less than $1 \mathrm{keV}$ (Sect. 5.1), which is too low to excite iron K-shell electrons, so that no emission is expected. MCSNR J0547-6973 is one case where a hotter spectral component $(k T \sim 2.2 \mathrm{keV})$ is present but no Fe $\mathrm{K}$ emission is detected, likely because the emission measure of that component is too low, so that it is not detectable with current instruments. Even if a spectrally unresolved hot iron component exists in more LMC remnants, a further issue is again detectability. The LMC SNRs of Yamaguchi et al. (2014) have hard X-ray ( $2 \mathrm{keV}-8 \mathrm{keV})$ luminosities above $10^{35} \mathrm{erg} \mathrm{s}^{-1}$. There are only two other SNRs in the LMC above this level, MCSNR J0540-6920 and N157B, which are powered by a bright pulsar and pulsar wind nebula, respectively.

Despite these observational difficulties, it is very likely that the sample of LMC Fe K-emitting remnants (of Yamaguchi et al. 2014, plus DEM L71) is complete, because all young SNRs ( $\$ 5000$ yr old) are now known and observed in X-rays. Translating the fraction of remnants with $\mathrm{Fe} \mathrm{K}$ emission in the LMC $(\approx 13 \%)$ to the Galactic population (294 objects, Green 2014), we expect more than 40 such sources in the Milky Way. This number is a lower limit, since fainter line fluxes than in the LMC can be reached. Yamaguchi et al. (2014) list 16 Galactic SNRs detected, out of 56 objects observed with Suzaku (Ferrand $\&$ Safi-Harb 2012, online database ${ }^{13}$ ). About 80 more SNRs were observed and detected with Chandra or XMM-Newton, and 150 have not been covered in X-rays. A systematic analysis of all X-ray-detected SNRs and new/deeper observations of promising candidates with more sensitive instruments (e.g. XMM-Newton vs. Chandra, future missions such as Athena) will provide a better census of $\mathrm{Fe} \mathrm{K}$ lines in SNRs. This will allow us to type more remnants and to study the pre-SN evolution of their progenitors.

\subsection{Detection of SN ejecta}

When SN ejecta give an observable contribution to the X-ray emission of an SNR, the fitted abundances, or rather the fitted abundance ratios, will reflect the nucleosynthesis yields of either thermonuclear or CC SNe. To identify SNRs with detected ejecta and the origin thereof, we computed abundance ratios $\mathrm{X} / \mathrm{Fe}$, where $\mathrm{X}$ is $\mathrm{O}, \mathrm{Ne}, \mathrm{Mg}$, or $\mathrm{Si}$. The ratios are normalised with respect to $(\mathrm{X} / \mathrm{Fe})_{\mathrm{LMC}}$, the corresponding ratios with the LMC abundances (from Russell \& Dopita 1992). As CC-SNRs produce large amounts of light- $Z$ elements and little iron, high $(\mathrm{X} / \mathrm{Fe}) /(\mathrm{X} / \mathrm{Fe})_{\mathrm{LMC}}$ ratios (in excess of one) indicate a massive star progenitor. On the contrary, the main product of thermonuclear SNe is iron, and ejecta in type Ia SNRs (if detected), are expected to have $(\mathrm{X} / \mathrm{Fe}) /(\mathrm{X} / \mathrm{Fe})_{\mathrm{LMC}} \ll 1$.

In Fig. 4, the abundance ratio diagrams of all SNRs with corresponding fitted abundances are shown. The samples of SNRs with a secured $\mathrm{CC}$ or type Ia classification (as described in Appendix B) are marked. Evidently, many of the known CC SNRs are located in regions of super-LMC X/Fe. The known type Ia SNRs are unsurprisingly in the $(\mathrm{X} / \mathrm{Fe}) /(\mathrm{X} / \mathrm{Fe})_{\mathrm{LMC}} \ll 1$ regions of the diagrams, because in most cases it is this very iron-enhancement that was used to classify them.

Several sources without previous classification are located in the high- and low-ratio regions of the diagrams. For typing purpose, we assign "high $\mathrm{X} / \mathrm{Fe}$ " and "low $\mathrm{X} / \mathrm{Fe}$ " flags to these objects, using the following scheme: For each element $\mathrm{X}$, we plot the cumulative distribution of the ratio $(\mathrm{X} / \mathrm{Fe}) /(\mathrm{X} / \mathrm{Fe})_{\mathrm{LMC}}$.

\footnotetext{
${ }^{13}$ http://www.physics. umanitoba.ca/snr/SNRcat/
}
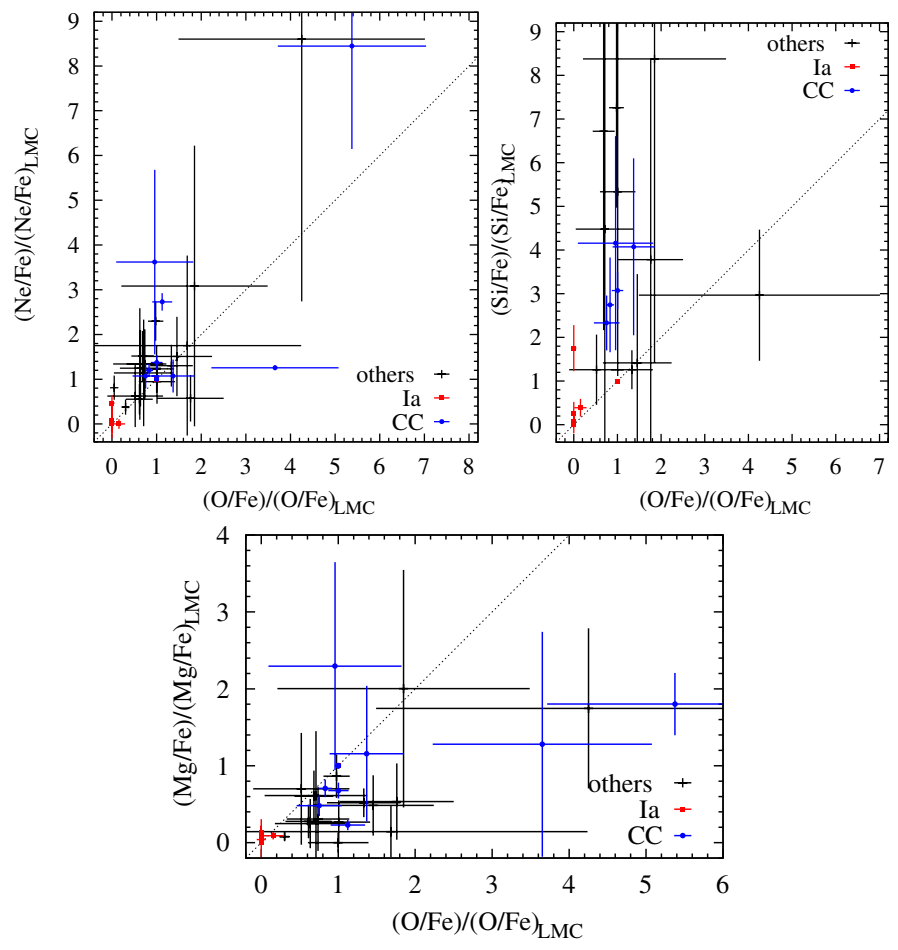

Fig. 4. Abundance ratio diagrams of LMC SNRs with fitted abundances. Sources firmly classified as type Ia or CC-SNRs are plotted in red and blue, respectively, and the rest of the sample in black. See text (Sect. 5.5) for details.

Table 3. Constraints used for the identification of ejecta in SNR spectra.

\begin{tabular}{ccccc}
\hline \hline $\mathrm{X}$ & \multicolumn{2}{c}{ "high X/Fe" flag } & \multicolumn{2}{c}{ "low X/Fe" flag } \\
& $(1)$ & $(2)$ & $(1)$ & $(3)$ \\
\hline $\mathrm{O}$ & $>1.0$ & $>0.83$ & $<0.60$ & $<0.83$ \\
$\mathrm{Ne}$ & $>1.43$ & $>1.30$ & $<0.55$ & $<1.30$ \\
$\mathrm{Mg}$ & $>0.62$ & $>0.48$ & $<0.22$ & $<0.48$ \\
$\mathrm{Si}$ & $>2.70$ & $>1.60$ & $<1.30$ & $<1.60$ \\
\hline
\end{tabular}

Notes. (1) Constraints on the ratio $(\mathrm{X} / \mathrm{Fe}) /(\mathrm{X} / \mathrm{Fe})_{\mathrm{LMC}}$. (2) Constraints on the lower limit $(\mathrm{X} / \mathrm{Fe}) /(\mathrm{X} / \mathrm{Fe})_{\mathrm{LMC}}-\Delta(\mathrm{X} / \mathrm{Fe})$, where $\Delta(\mathrm{X} / \mathrm{Fe})$ is the uncertainty of the abundance ratio. (3) Constraints on the upper limit $(\mathrm{X} / \mathrm{Fe}) /(\mathrm{X} / \mathrm{Fe})_{\mathrm{LMC}}+\Delta(\mathrm{X} / \mathrm{Fe})$.

We then assign a "high $\mathrm{X} / \mathrm{Fe}$ " flag to an object if its ratio is above the 68th percentile $(\sim 1 \sigma)$ of the cumulative distribution. Symmetrically, a "low X/Fe" flag is given if the ratio is below the 32th percentile. Since the uncertainties in the fitted abundances can be large, it is necessary to put a second constraint using the uncertainty of the ratio, noted $\Delta(\mathrm{X} / \mathrm{Fe})$ : a "high $\mathrm{X} / \mathrm{Fe}$ " flag is only given if the lower limit (i.e. ratio minus the uncertainty) is above the median of the cumulative distribution. For a "low X/Fe" flag the upper limit must be below the median. This excludes all cases where the ratios are elevated (or much smaller than one) but highly uncertain. Though some of the criteria for "low X/Fe" flags may seem high, the selected SNRs have actual ratios well below half the LMC average (well below 0.2 times the average for $\mathrm{Mg}$ ). The criteria for "high $\mathrm{X} / \mathrm{Fe}$ " and "low X/Fe" flag are given in Table 3. There are 23 SNRs in the $1 \mathrm{~T} / 2 \mathrm{~T}$ sample with high or low abundance ratio flags, as listed in Table 4. These flags are used in Sect. 6 to help the typing of all LMC SNRs. 
Table 4. 1T/2T SNRs with detected ejecta (top part), and used for measurements of ISM composition (bottom part).

\begin{tabular}{|c|c|c|c|c|c|c|c|c|c|c|}
\hline \multirow{2}{*}{ MCSNR } & \multirow{2}{*}{ Old name } & \multirow{2}{*}{ SN type } & \multicolumn{4}{|c|}{ High $\mathrm{X} / \mathrm{Fe}$ flags } & \multicolumn{4}{|c|}{ Low $\mathrm{X} / \mathrm{Fe}$ flags } \\
\hline & & & $\mathrm{O}$ & $\mathrm{Ne}$ & $\mathrm{Mg}$ & $\mathrm{Si}$ & $\mathrm{O}$ & $\mathrm{Ne}$ & $\mathrm{Mg}$ & $\mathrm{Si}$ \\
\hline J0453-6829 & В0453-685 & $\mathrm{CC}$ & - & - & $\mathrm{Y}$ & $\mathrm{Y}$ & - & - & - & - \\
\hline J0506-6541 & - & & - & Y & - & - & - & - & - & - \\
\hline J0506-7026 & [HP99] 1139 & & - & - & - & - & $\mathrm{Y}$ & $\mathrm{Y}$ & $\mathrm{Y}$ & - \\
\hline J0508-6830 & - & Ia & - & - & - & - & Y & - & - & - \\
\hline J0508-6902 & [HP99] 791 & Ia & - & - & - & - & $\mathrm{Y}$ & - & - & - \\
\hline J0511-6759 & - & Ia & - & - & - & - & $\mathrm{Y}$ & - & - & - \\
\hline J0519-6926 & В0520-694 & & - & Y & Y & $\mathrm{Y}$ & - & - & - & - \\
\hline J0523-6753 & N44 & & $\mathrm{Y}$ & Y & Y & - & - & - & - & - \\
\hline J0525-6559 & N49B & $\mathrm{CC}$ & - & Y & $\mathrm{Y}$ & $\mathrm{Y}$ & - & - & - & - \\
\hline J0526-6605 & N49 & $\mathrm{CC}$ & $\mathrm{Y}$ & - & Y & Y & - & - & - & - \\
\hline J0529-6653 & DEM L214 & & $\mathrm{Y}$ & - & - & - & - & - & - & - \\
\hline J0531-7100 & N206 & & $\mathrm{Y}$ & - & - & $\mathrm{Y}$ & - & - & - & - \\
\hline J0533-7202 & 1RXSJ053353.6-7204 & & - & - & - & - & - & - & Y & - \\
\hline J0534-6955 & B0534-699 & Ia & - & - & - & - & $\mathrm{Y}$ & $\mathrm{Y}$ & Y & - \\
\hline J0534-7033 & DEM L238 & Ia & - & - & - & - & $\mathrm{Y}$ & $\mathrm{Y}$ & Y & $\mathrm{Y}$ \\
\hline J0535-6602 & N63A & $\mathrm{CC}$ & $\mathrm{Y}$ & Y & - & - & - & - & - & - \\
\hline J0535-6918 & Honeycomb & & - & - & - & Y & - & - & - & - \\
\hline J0536-6735 & DEM L241 & $\mathrm{CC}$ & $\mathrm{Y}$ & Y & $\mathrm{Y}$ & - & - & - & - & - \\
\hline J0536-6913 & B0536-6914 & $\mathrm{CC}$ & Y & - & - & - & - & - & - & - \\
\hline J0536-7039 & DEM L249 & Ia & - & - & - & - & $\mathrm{Y}$ & $\mathrm{Y}$ & Y & $\mathrm{Y}$ \\
\hline J0537-6628 & DEM L256 & & - & - & - & - & - & - & $\mathrm{Y}$ & - \\
\hline J0547-6941 & DEM L316A & Ia & - & - & - & - & Y & $\mathrm{Y}$ & $\mathrm{Y}$ & $\mathrm{Y}$ \\
\hline \multirow[t]{3}{*}{ J0547-7025 } & B0548-704 & Ia & - & - & - & - & $\mathrm{Y}$ & $\mathrm{Y}$ & $\mathrm{Y}$ & - \\
\hline & & & \multicolumn{8}{|c|}{ ISM abundance } \\
\hline & & & \multicolumn{2}{|c|}{$\mathrm{O} \& \mathrm{Fe}$} & \multicolumn{2}{|c|}{$\mathrm{Ne}$} & \multicolumn{2}{|c|}{$\mathrm{Mg}$} & \multicolumn{2}{|c|}{$\mathrm{Si}$} \\
\hline J0450-7050 & В0450-709 & & \multicolumn{2}{|c|}{$\mathrm{Y}$} & \multicolumn{2}{|c|}{ Y } & \multicolumn{2}{|c|}{$\mathrm{Y}$} & \multicolumn{2}{|c|}{-} \\
\hline J0453-6655 & N4 & & \multicolumn{2}{|c|}{$\mathrm{Y}$} & \multicolumn{2}{|c|}{ Y } & \multicolumn{2}{|c|}{$\mathrm{Y}$} & \multicolumn{2}{|c|}{ - } \\
\hline J0453-6829 & В0453-685 & $\mathrm{CC}$ & \multicolumn{2}{|c|}{ Y } & \multicolumn{2}{|c|}{$\mathrm{Y}$} & & - & \multicolumn{2}{|c|}{ - } \\
\hline J0454-6626 & N11L & & & Y & Y & & & $Y$ & - & \\
\hline J0505-6802 & $\mathrm{N} 23$ & $\mathrm{CC}$ & & Y & Y & & & Y & $Y$ & \\
\hline J0514-6840 & - & & & Y & - & & & - & - & \\
\hline J0518-6939 & N120 & & & Y & Y & & & Y & $Y$ & \\
\hline J0519-6926 & В0520-694 & & & Y & - & & & - & - & \\
\hline J0527-6912 & B0528-692 & & & Y & $Y$ & & & Y & $Y$ & \\
\hline J0528-6727 & DEM L205 & & & Y & - & & & - & - & \\
\hline J0531-7100 & N206 & $\mathrm{CC}$ & & - & Y & & & Y & - & \\
\hline J0532-6732 & B0532-675 & & & Y & $Y$ & & & Y & $Y$ & \\
\hline J0533-7202 & 1RXSJ053353.6-7204 & & & Y & Y & & & - & - & \\
\hline J0535-6918 & Honeycomb & & & Y & Y & & & Y & - & \\
\hline J0543-6858 & DEM L299 & & & Y & $\mathrm{Y}$ & & & Y & $Y$ & \\
\hline J0547-6943 & DEM L316B & & & Y & Y & & & Y & $Y$ & \\
\hline
\end{tabular}

Notes. The classification given (type Ia or core-collapse) is described in Appendix B.

\subsection{Metal abundances of the LMC ISM}

When no SN ejecta is detected, the X-ray emission is dominated by the ISM swept-up by the SN blast wave. Therefore, the fitted abundances in these cases provide us with measurements of the chemical composition of the gas phase of the LMC ISM. Russell \& Dopita (1992) and Hughes et al. (1998) have used samples of SNRs to obtain the abundance of some elements (using optical and X-ray observations, respectively), but the smaller sample of known SNRs and sensitivity of the X-ray instrument used $(A S C A)$ at the time limited the number of SNRs eligible to measure LMC abundances.

We first selected all 1T/2T SNRs with fitted abundances but no high or low abundance ratios. To increase that sample, we included SNRs where some abundances are enhanced but others can still be used. For example in MCSNR J0453-6829, the spectrum is enhanced in $\mathrm{Mg}$ and $\mathrm{Si}$, but the fitted values for $\mathrm{O}, \mathrm{Ne}$, and Fe, are still (assumed to be) reflecting the LMC ISM abundance. Furthermore, if the abundance of a given element is too uncertain, then the SNR is not used to measure the average abundance of that element. This limits in particular the size of the SNR sample allowing the abundance of silicon to be measured.

In Table 4 we give the list of SNRs used to measure the abundance of $\mathrm{O}, \mathrm{Ne}, \mathrm{Mg}, \mathrm{Si}$, and $\mathrm{Fe}$, or a subset of these elements. The measured abundances for this sample are plotted relative to solar values in Fig. 5. The final LMC abundances are obtained by taking the average values from all SNRs where an element is used; the errors given are the RMS scatter amongst the SNRs used. This method is similar to that of Hughes et al. (1998). Resulting abundances range from $\sim 0.2$ solar for oxygen to $\sim 0.7$ solar for silicon. The results are listed in Table 5. The absolute abundances, in the form $12+\log (\mathrm{X} / \mathrm{H})$ (by number), are given, in comparison with results from Russell \& Dopita (1992) 


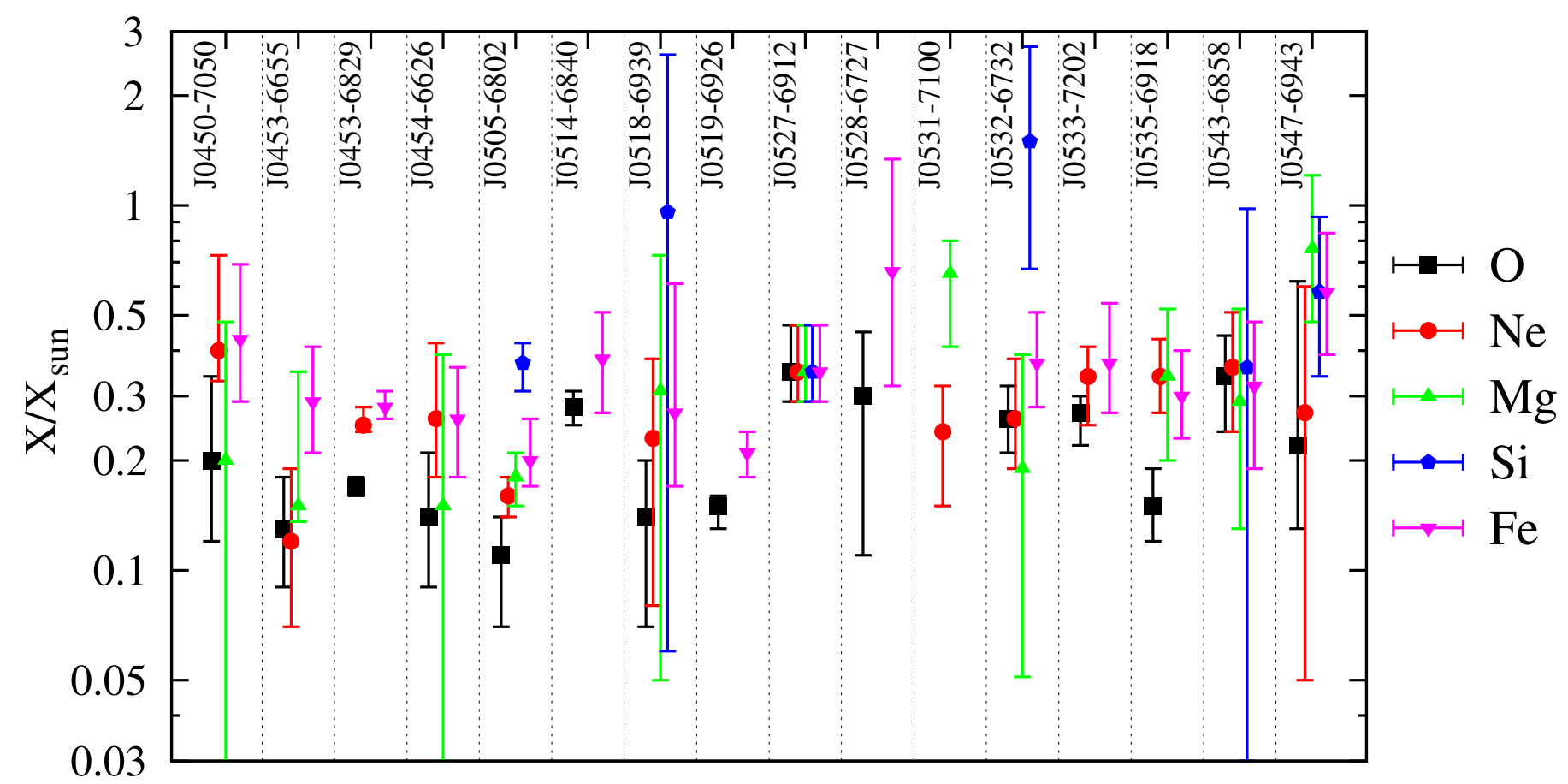

Fig. 5. LMC ISM abundances, relative to the solar values of Wilms et al. (2000), measured in a sample of 16 X-ray SNRs. The selection of the sample and the measurements of abundance are described in Sect. 5.6.

Table 5. LMC abundances.

\begin{tabular}{ccccccc}
\hline \hline Element & $\begin{array}{c}\mathrm{X} / \mathrm{X}_{\odot} \\
(1)\end{array}$ & $\begin{array}{c}N \\
(2)\end{array}$ & $\begin{array}{c}\mathrm{rms} \\
(3)\end{array}$ & $12+\log (\mathrm{X} / \mathrm{H})$ & $\begin{array}{c}\text { Hughes et al. } \\
(1998)\end{array}$ & $\mathrm{RD} 92$ \\
\hline $\mathrm{O}$ & 0.21 & 15 & 0.08 & $8.01_{-0.21}^{+0.14}$ & $8.21 \pm 0.07$ & $8.35 \pm 0.06$ \\
$\mathrm{Ne}$ & 0.28 & 13 & 0.08 & $7.39_{-0.15}^{+0.11}$ & $7.55 \pm 0.08$ & $7.61 \pm 0.05$ \\
$\mathrm{Mg}$ & 0.33 & 11 & 0.19 & $6.92_{-0.37}^{+0.20}$ & $7.08 \pm 0.07$ & $7.47 \pm 0.13$ \\
$\mathrm{Si}$ & 0.69 & 6 & 0.42 & $7.11_{-0.41}^{+0.20}$ & $7.04 \pm 0.08$ & $7.81^{a}$ \\
$\mathrm{Fe}$ & 0.35 & 15 & 0.12 & $6.97_{-0.18}^{+0.13}$ & $7.01 \pm 0.11$ & $7.23 \pm 0.14$ \\
\hline
\end{tabular}

Notes. (1) Abundance relative to the solar value of Wilms et al. (2000). (2) Number of SNRs used to measure $X / X_{\odot}$. (3) RMS scatter amongst the N SNRs. ${ }^{(a)}$ Silicon abundance was quoted as highly uncertain in Russell \& Dopita (1992).

and Hughes et al. (1998). Abundances of Fe and Si measured with XMM-Newton are in good agreement with the results measured for a different sample of SNRs by Hughes et al. (1998) ${ }^{14}$. More recent studies of abundances in the LMC, using large samples of field stars (Cole et al. 2005; Pompéia et al. 2008; Lapenna et al. 2012; Van der Swaelmen et al. 2013), can be used to evaluate our results. The metallicity distributions $[\mathrm{Fe} / \mathrm{H}]^{15}$ peak at about -0.5 dex for most field star samples (Lapenna et al. 2012), corresponding to $12+\log (\mathrm{Fe} / \mathrm{H})=7$. This matches very well our value based on XMM-Newton SNRs $\left(6.97_{-0.18}^{+0.13}\right)$, indicating no metallicity difference between field stars and gas-phase ISM.

However, the abundances of light $\alpha$-elements tend to be lower (by $\sim 0.15$ dex -0.2 dex) compared to Hughes et al. (1998), although the results for $\mathrm{Mg}$ and $\mathrm{Ne}$ might still be reconciled given the larger uncertainties. Still, we measured a ratio $[\mathrm{O} / \mathrm{Fe}]$ of -0.21 while $A S C A$ SNRs gave -0.06 . The likely explanation is two-fold. First, the $\alpha$-elements abundance has an intrinsic

\footnotetext{
${ }_{14}$ They used six CC SNRs (J0453-6829, N23, N49, N49B, N63A, and N132D) and one type Ia SNR (DEM L71).

${ }^{15}$ Using the conventional notation: $[\mathrm{X} / \mathrm{Y}]=\log (\mathrm{X} / \mathrm{Y})-\log (\mathrm{X} / \mathrm{Y})_{\odot}$.
}

scatter (about 0.05 dex -0.08 dex at the relevant metallicity, Van der Swaelmen et al. 2013) that can partly explain the discrepancy. The second reason is the sample used by Hughes et al. (1998): N132D, N49, and N49B contain regions that have been (since then) found to be enhanced in low-Z elements (e.g. this work, and references in Appendix B), while the bright central regions of the type Ia SNR DEM L71 are enriched in iron. These contributions from ejecta to the integrated spectra measured with ASCA affect the measured LMC abundances. Six out of the seven SNRs used by Hughes et al. (1998) are well-established CC-SNRs, and this bias is likely to explain their higher $[\mathrm{O} / \mathrm{Fe}]$ (or more generally $[\alpha / \mathrm{Fe}]$ ). On the contrary, the XMM-Newton sample used here is explicitly cleaned of SNRs with abnormal abundance patterns (i.e. those with ejecta detected), resulting in a purer sample better suited to the measurement of the ISM composition. However, this sample comprises SNRs fainter than used in previous studies, and the abundances thus obtained are consequently relatively uncertain.

The abundance pattern of metals should reflect the past history of chemical enrichment, and in particular the relative number of CC and Ia SNRs (hereafter $N_{\mathrm{CC}} / N_{\mathrm{Ia}}$ ), because their metal yields are markedly different. In Fig. 6 we show the $[\mathrm{O} / \mathrm{Fe}]$ and $[\mathrm{Mg} / \mathrm{Fe}]$ vs. $[\mathrm{Fe} / \mathrm{H}]$ diagrams. Abundances measured with SNRs (i.e. that of the ISM gas phase) are compared with that measured in older populations: old globular clusters from Johnson et al. (2006, ages $10 \mathrm{Gyr}$ ) and Bar and disc field red giant stars from Van der Swaelmen et al. (2013, ages $\gtrsim 1$ Gyr). SNRs are only found in the higher metallicity $([\mathrm{Fe} / \mathrm{H}])$ range. There is also a clear trend for SNRs to be at lower $[\alpha / \mathrm{Fe}]$ ratios, although uncertainties from X-ray spectral fitting are large (particularly for $[\mathrm{Mg} / \mathrm{Fe}])$. A larger sample and more data would be desirable to demonstrate this result definitely. Nevertheless, this trend should reflect the continued enrichment by type Ia SNe in the last $\sim 1$ Gyr, which inject large amounts of Fe back in the ISM and drive younger populations towards the bottom right corner of the $[\alpha / \mathrm{Fe}]-[\mathrm{Fe} / \mathrm{H}]$ diagrams. 


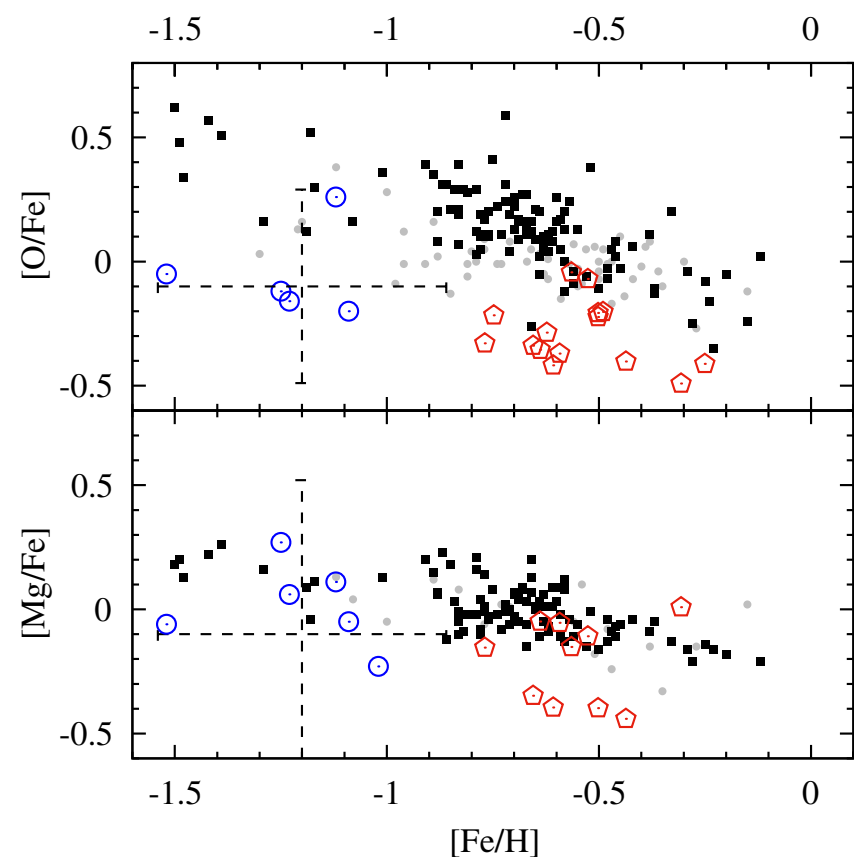

Fig. 6. $[\mathrm{O} / \mathrm{Fe}]$ and $[\mathrm{Mg} / \mathrm{Fe}]$ vs. $[\mathrm{Fe} / \mathrm{H}]$ diagrams for various $\mathrm{LMC}$ populations: abundances measured in SNRs (ISM gas phase, this work) are shown with red pentagons. The crosses indicate median error bars. Blue open circles are the old globular clusters from Johnson et al. (2006, ages $\sim 10 \mathrm{Gyr})$. Chemical abundances of Bar and disc stars are marked by black squares and grey dots, respectively (from Van der Swaelmen et al. 2013, ages $\gtrsim 1 \mathrm{Gyr}$ ).

There are SNR-to-SNR variations in the abundances, but the metallicity scatter in the ISM gas phase is less than for field stars. In particular there is no metal-poor population $([\mathrm{Fe} / \mathrm{H}] \lesssim-0.9)$. We also checked that there is no clear correlation between the location of an SNR in the $[\alpha / \mathrm{Fe}]-[\mathrm{Fe} / \mathrm{H}]$ diagrams and the SFH around the SNR. For instance, SNRs with relatively high $[\alpha / \mathrm{Fe}]$ are not necessarily in regions with increased recent SF, which would produce massive stars that release low-Z elements. Despite the uncertainties and the limited size of the sample, this lack of correlation likely indicates that $\mathrm{SNe}$-produced elements are well mixed in the ISM. In other words, the ISM is quickly homogenised, at least at the spatial scales over which SFH is measured $(\sim 200 \mathrm{pc})$.

After LMC abundances were measured (e.g. Russell \& Dopita 1992), Tsujimoto et al. (1995) found with chemical evolution models that the deficit of light $\alpha$-elements of the MCs (i.e. lower $[\alpha / \mathrm{Fe}]$ for a given $[\mathrm{Fe} / \mathrm{H}])$ compared to the Galaxy must be explained by a smaller $N_{\mathrm{CC}} / N_{\mathrm{Ia}}$ (more type Ia $\mathrm{SNe}$ ). They measured a Galactic ratio of 6.7 , but $N_{\mathrm{CC}} / N_{\mathrm{Ia}} \sim 4-5$ and $\sim 3.3$ for the LMC and SMC, respectively. Our results for the LMC ISM abundance suggest an even lower ratio $N_{\text {CC }} / N_{\text {Ia }}$, because the deficit of light $\alpha$-elements is wider than previously assumed by Tsujimoto et al. (1995). By tentatively typing all LMC remnants, we show in Sect. 6 that indeed $N_{\mathrm{CC}} / N_{\text {Ia }}$ is particularly low, compared to previous measurements of the ratio in the LMC or inferred from galaxy cluster X-ray observations, and discuss likely explanations.

\section{Measuring the ratio of $\mathrm{CC}$ to type la $\mathrm{SNe}$ in the LMC using "SFH-typing"}

\subsection{The typing of SNRs in general}

Because the two flavours of $\mathrm{SNe}$ deposit a similar amount of energy in the ISM, they produce remnants which become increasingly hard to type as they age. The most secured typing methods are the study of SN optical light echoes (Rest et al. 2005, 2008; infrared light echoes can be used to probe the ISM dust, see e.g. Vogt et al. 2012), the measurement of the nucleosynthesis products in the ejecta (e.g. Hughes et al. 1995), or the association with a neutron star/pulsar wind nebula. Optical spectroscopy can also be used: In some cases the fast-moving ejecta are detected in optical lines with highly elevated abundances of oxygen. Those so-called oxygen-rich SNRs have massive star progenitors, see for instance Lasker (1978), Chevalier \& Kirshner (1979), Morse et al. (1995), and references therein. On the contrary, some SNRs have prominent Balmer lines of hydrogen, but absent or weak [S II] and [O III] lines. These Balmerdominated optical spectra are interpreted as non-radiative shocks overtaking (partially) neutral gas (Chevalier \& Raymond 1978; Chevalier et al. 1980). A type Ia SN progenitor is consistent with the presence of neutral gas, as massive stars would ionise their surrounding. A sample of optically bright Balmer-dominated SNRs was detected in the LMC by Tuohy et al. (1982).

These methods work best for relatively young remnants ( $\$ 5000 \mathrm{yr}$ ), leaving a significant fraction of the SNR population untyped. However, several evolved SNRs have been discovered (in X-rays) in the Magellanic Clouds with an iron-rich, centrally bright emission (Nishiuchi et al. 2001; Hendrick et al. 2003; van der Heyden et al. 2004; Seward et al. 2006; Borkowski et al. 2006; Bozzetto et al. 2014; Maggi et al. 2014), naturally leading to their classification as type Ia remnants.

In addition, studies of the X-ray and infrared morphologies of SNRs (Lopez et al. 2009; Peters et al. 2013) suggest that, as a class, type Ia and CC SNRs have distinct symmetries: type Ia remnants are statistically more spherical and mirror symmetric than the CC SNRs. However, this method cannot give definite results for individual objects: Prominent counterexamples include SNR 1E 0102.2-7219, a textbook CC SNR which is highly symmetric (Flanagan et al. 2004), and MCSNR J0547-7025, which is a type Ia SNR (based on its X-ray spectrum Hendrick et al. 2003, this work) with "anomalous" ejecta distribution (Lopez et al. 2009).

For a decent fraction of the LMC remnants, these various methods give a secured classification. This "secured-type" sample is presented in Appendix B and listed in Table A.1. To tentatively type the rest of the sample, we devise a new way to quantify the local stellar environment of LMC, as described in Sect. 6.2. This method is calibrated with the "secured-type" sample and applied in Sect. 6.3. We can then discuss the measured ratio of CC to type Ia SNRs in the LMC and its implications in Sect. 6.4.

\subsection{Evaluating the local stellar environment}

We devised two metrics to assess the local stellar environment of LMC SNRs. Both ultimately stem from the same set of data (the MCPS catalogue of Zaritsky et al. 2004). Although connected, they still measure two distinct properties and are therefore complementary, as we discuss below. The two metrics are given for each SNR in Table C.1.

- $N_{\mathrm{OB}}$, the number of blue early-type stars in the immediate vicinity of the remnant:

To obtain this number, we constructed a $V$ vs. $(B-V)$ colour-magnitude diagram (CMD) of all stars whose projected position lies within $100 \mathrm{pc}\left(\sim 6.9^{\prime}\right)$ of each SNR. This value corresponds to the drift distance for a star of age $10^{7} \mathrm{yr}$ at a velocity of $10 \mathrm{~km} \mathrm{~s}^{-1}$ and was used by 

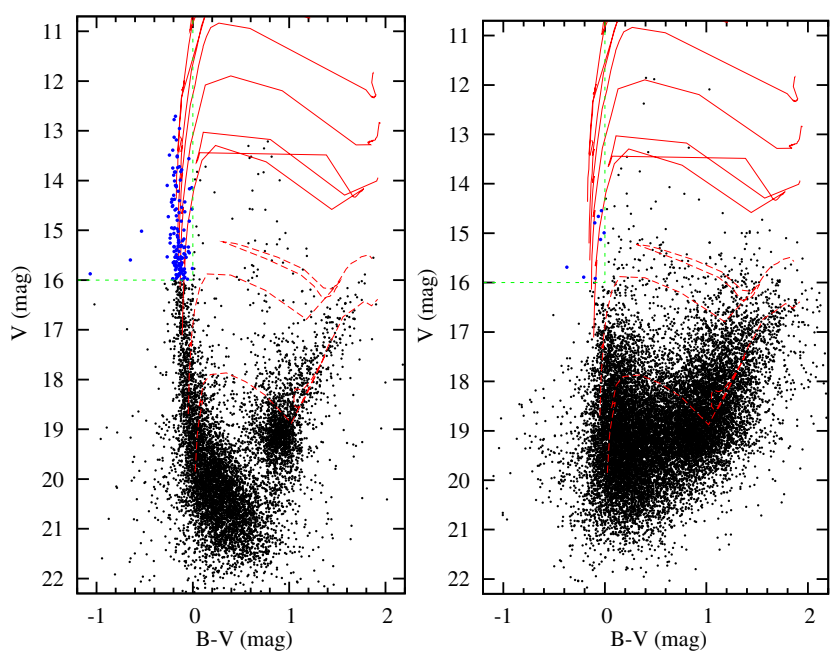

Fig. 7. Colour-magnitude diagram (CMD) of the MCPS stars (Zaritsky et al. 2004) within $100 \mathrm{pc}\left(\sim 6.9^{\prime}\right)$ of the central position of two remnants, MCSNR J0528-6727 (left) and MCSNR J0534-6955 (right). Geneva stellar evolution tracks (Lejeune \& Schaerer 2001) are shown as red lines, for metallicity of $0.4 Z_{\odot}$ and initial masses of $3,5 M_{\odot}$ (dashed lines) and $10,15,20,25$, and $40 M_{\odot}$ (solid lines), from bottom to top. The green dashed line shows the criteria used to identify the OB stars $(V<16$ and $B-V<0$ ). Stars satisfying these criteria are shown as blue dots.

Chu \& Kennicutt (1988). The upper main-sequence of stars in the LMC was identified by adding the stellar evolutionary tracks of Lejeune \& Schaerer (2001), for $Z=0.4 Z_{\odot}$ and initial masses from $3 M_{\odot}$ to $40 M_{\odot}$. We assumed a distance modulus of 18.49 and an extinction $A_{V}=0.5$ (the average extinction for "hot" stars, Zaritsky et al. 2004). From there, we used the criteria of $V<16$ and $B-V<$ 0 to identify OB stars. In Fig. 7 we show two example CMDs of the regions around MCSNR J0528-6727 (left) and MCSNR J0534-6955 (right). In the former case, a prominent upper-main sequence is obvious and the number of OB stars $N_{\mathrm{OB}}=142$. By contrast, the region around MCSNR J0534-6955 is devoid of young massive stars. For this remnant, $N_{\mathrm{OB}}$ is only 8 . Note that $N_{\mathrm{OB}}$ is likely a lower limit on the actual number of massive stars due to stellar crowding (the typical seeing of the MCPS is $1.5^{\prime \prime}$ ). This issue affects chiefly regions with higher $N_{\mathrm{OB}}$.

- $r=N_{\mathrm{CC}} / N_{\text {Ia }}$, the ratio of CC SNe to thermonuclear SNe expected from the observed distribution of stellar ages in the neighbourhood of the remnants:

This number is obtained via the spatially resolved SFH map of Harris \& Zaritsky (2009, see Sect. 2.3). For each SNR we plot the SFR of the cell including the remnant as a function of lookback time and metallicity. Two example SFHs are shown in Fig. 8 for the same SNRs of Fig. 7. They are strikingly different: the SFR around J0528-6727 soared in the last $20 \mathrm{Myr}$, when the numerous early-type stars in the vicinity of the remnant were formed, while the star formation around J0534-6955 peaked (at a lower absolute rate) about $125 \mathrm{Myr}$ ago and was shut down in the most recent 20 Myr.

Because stars might drift away from their birth place, one potentially important caveat is that the SFH of a cell hosting an SNR may be derived from stars having no physical connection with the SNR progenitor. For a detailed discussion on the relevance of local stellar populations to the study of progenitors, we point
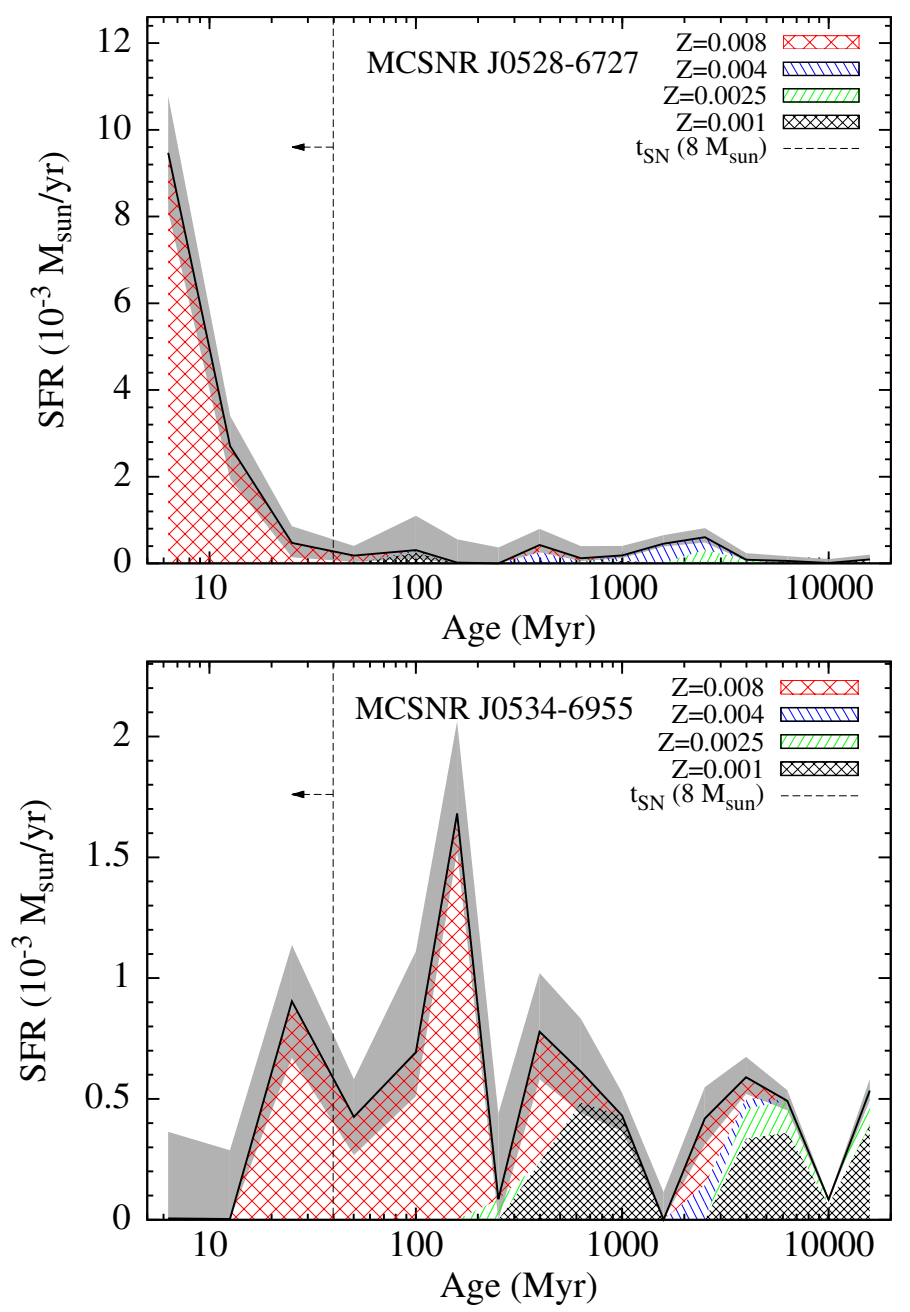

Fig. 8. Star formation history around MCSNR J0528-6727 (top) and J0534-6955 (bottom). Data are taken from Harris \& Zaritsky (2009). The star formation rate in four metallicity bins are plotted against lookback time. The errors (combining all metallicities) are shown by the grey shading. The vertical dashed line at $40 \mathrm{Myr}$ indicates the maximal lifetime of a CC SN progenitor. Note the changing vertical scale.

to Badenes et al. (2009). However, we stress that most of the information that can be gained from the study of the local SFHs, in the context of typing remnants, is contained in the most recent time bins. Namely, the presence of recent star formation episode is a strong necessary (but not sufficient) condition to tentatively type a remnant as having a CC origin. Conversely, the lack of recent star-forming activity favours a thermonuclear origin.

To approach this question in a quantitative way, we did the following: We used the delay time distribution (DTD) $\Psi_{i}(\tau)$, the $\mathrm{SN}$ rate at time $\tau$ following a star formation event, measured by Maoz \& Badenes (2010) in the Magellanic Clouds, with $i=1$, 2 , and 3 designating the time intervals they used $(t<35 \mathrm{Myr}$, $35 \mathrm{Myr}<t<330 \mathrm{Myr}$, and $330 \mathrm{Myr}<t<14 \mathrm{Gyr}$, respectively). From timescale arguments it is reasonably assumed that $\Psi_{1}$ will correspond to the CC-SN rate, whilst $\Psi_{2}$ and $\Psi_{3}$ will be that of SNe Ia (regardless of their "prompt" or "delayed" nature). The SFR is integrated to obtain $M_{i}$, the stellar mass formed in each time interval. The SFH of Harris \& Zaritsky (2009) is only given at $t=25 \mathrm{Myr}$ and $t=50 \mathrm{Myr}$. To obtain $M_{1}$, the mass formed at $t<35 \mathrm{Myr}$, we approximate $M(25<t<35)$ as half that formed between $25 \mathrm{Myr}$ and $50 \mathrm{Myr}$ (the second half is included in $M_{2}$ ). 

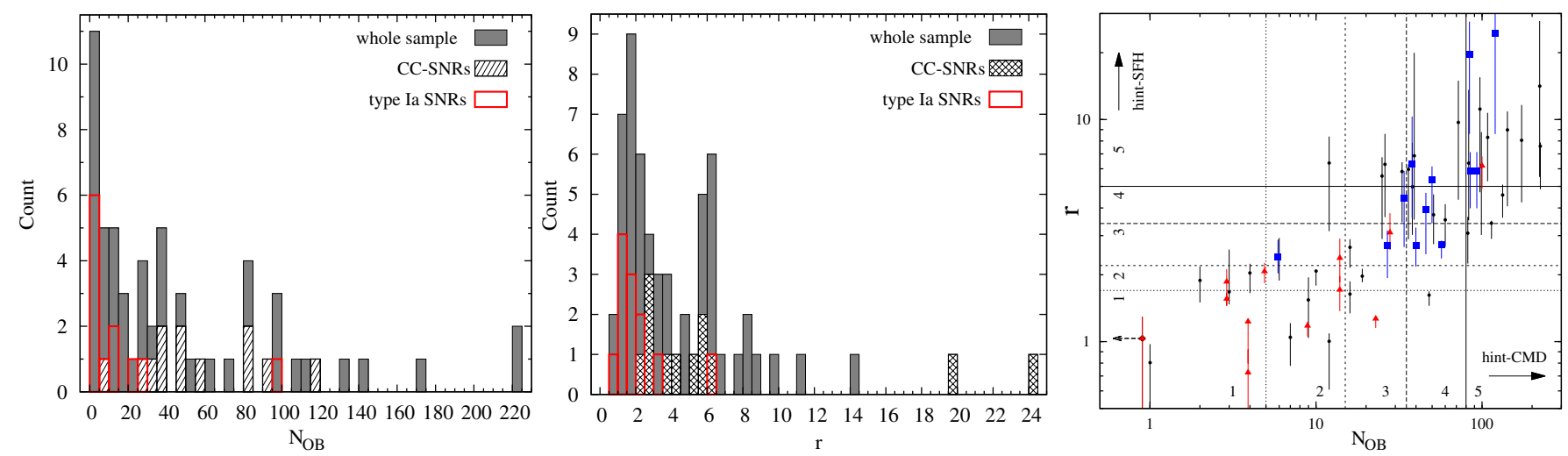

Fig. 9. Left and middle panel: count distribution of LMC SNRs as function of $N_{\mathrm{OB}}$ and $r$. The distribution for the SNRs with a secured CC classification is shown with the hatched boxes; that for type Ia SNRs is outlined in red. The whole sample is shown by the solid grey histograms. Right panel: $r-N_{\mathrm{OB}}$ diagram of LMC SNRs. Secured Ia and CC SNRs are marked by red triangles and blue squares, respectively; the rest of the sample is shown with black dots. The arrow in the lower left corner indicates an SNR with $N_{\mathrm{OB}}=0$. The regions corresponding to different "Hint-SFH" and "Hint-CMD" are marked by the gridding.

Likewise, we split the mass formed between $t=250 \mathrm{Myr}$ and $t=400 \mathrm{Myr}$ in two and include a half in both $M_{2}$ and $M_{3}$.

Then, we compute $r=N_{\mathrm{CC}} / N_{\text {Ia }}$ as the ratio of the rates of $\mathrm{CC}$ and Ia $\mathrm{SNe}$, since the visibility times are the same for both types, i.e.:

$$
r=\frac{\Psi_{1} M_{1}}{\Psi_{2} M_{2}+\Psi_{3} M_{3}}
$$

Over the visibility time of a remnant - taking $100 \mathrm{kyr}$ as a very conservative limit - the stars in the SFH cell including the remnant will not drift away. In other words, the distribution of stellar ages observed now is the same as that when the SN exploded. $r$ is therefore a measure of the relative size of the pool of possible progenitors of both types. Using the same example SNRs as in Fig. 8, a value of $r=9.0_{-4.9}^{+1.9}$ is obtained for J0528-6727 while for J0534-6955 it is only $r=1.2 \pm 0.1$.

\section{3. "SFH-typing" all LMC SNRs}

We now proceed to give a tentative type to the whole sample of SNRs in the LMC, using $N_{\mathrm{OB}}$ and $r$. We assign two numbers called "Hint-CMD" and "Hint-SFH", depending on the $N_{\mathrm{OB}}$ and $r$-value obtained for each SNR, respectively. The numbers range from 1 meaning "strongly favours a type Ia SN origin", to 5 meaning "strongly favours a CC-SN origin". We used the distribution of $N_{\mathrm{OB}}$ and $r$ for the sample of "secured-type" SNR to establish the correspondence between their values and the hints.

This method is conceptually similar to that used by Chu \& Kennicutt (1988), albeit with several improvements: firstly, the sample in this work is twice the size of that available to Chu \& Kennicutt. Secondly, many ( 25) SNRs have now a secured type (Appendix B) and can be used to calibrate the method and evaluate the rate of erroneous classification. Then, the completeness of the census of early-type stars in the vicinity of the remnants is higher, owing to the use of the MCPS catalogue. Finally,

\footnotetext{
${ }^{16}$ The uncertainty given for $r$ solely includes that of the mass formed $M_{i}$, which is computed from uncertainties of the SFR given in Harris \& Zaritsky (2009). The uncertainties on $\Psi_{2}$ and $\Psi_{3}$ are larger, but are the same for all SNRs in the sample, allowing the use of $r$ in a comparative fashion. we adopted $\Psi_{2}=0.26 \mathrm{SNe} \mathrm{yr}^{-1}\left(10^{10} M_{\odot}\right)^{-1}$ and $\Psi_{3}<0.0014$ $\mathrm{SNe} \mathrm{yr}^{-1}\left(10^{10} M_{\odot}\right)^{-1}$. Note that because $\Psi_{3}$ is an upper limit, $r$ is formally a lower limit.
}

Table 6. Criteria and "Hints" attributed to SNRs as function of $N_{\mathrm{OB}}$ and $r$.

\begin{tabular}{cccc}
\hline \hline Value & Hint-CMD & Hint-SFH & Meaning \\
\hline 1 & $N_{\mathrm{OB}}<5$ & $r<1.7$ & Strongly favours a type Ia \\
2 & $5 \leq N_{\mathrm{OB}}<15$ & $1.7<r<2.2$ & Moderately favours a type Ia \\
3 & $15 \leq N_{\mathrm{OB}}<35$ & $2.2<r<3.4$ & Undecided \\
4 & $35 \leq N_{\mathrm{OB}}<80$ & $3.4<r<5$ & Moderately favours a CC \\
5 & $80 \leq N_{\mathrm{OB}}$ & $5<r$ & Strongly favours a CC \\
\hline
\end{tabular}

the spatially-resolved SFH reconstruction was simply unavailable before Harris \& Zaritsky (2009).

Calibration of the "SFH-typing": the number of OB stars in the vicinity of the secured type Ia and CC SNRs is shown in Fig. 9 (left panel). The two samples are rather well separated: the majority of type Ia SNRs have less than 20 early-type stars in their neighbourhood, while most of the CC-SNRs have $N_{\mathrm{OB}}>30$. The single major type Ia outlier is $\mathrm{N} 103 \mathrm{~B}\left(N_{\mathrm{OB}}=99\right)$, which is known to be in a region with a vigorous recent star formation activity (e.g. Badenes et al. 2009). MCSNR J0453-6829 is the only CC-SNRs to have a moderate $N_{\mathrm{OB}}(<25)$. The choice of "Hint-CMD" is given in Table 6 to reflect this distribution: $N_{\text {OB }}$ less than 5 (less than 15) strongly (moderately) favours a type Ia classification, while $N_{\mathrm{OB}}$ in excess of 80 (35) strongly (moderately) favours the CC-SN case.

Intuitively, any value $r>1$ should favour a CC SN origin (conversely for a thermonuclear origin). However, an important caveat in interpreting $r$ is that the rates of $\mathrm{Maoz} \&$ Badenes (2010), especially $\Psi_{2}$ and $\Psi_{3}$, are quite uncertain, due to the still limited sample of SNRs. Specifically, $\Psi_{2}$ has a value that changes by a factor of four depending on the tracer used to constrain the SNR visibility time. To provide a better feeling on what $r$-value to expect in either case (and to decide where is the separation), we show the count distribution of secured type Ia and CC SNRs in the $r$-domain in Fig. 9 (middle panel). There is a stronger overlap of both types in the intermediate range $(2.2 \lesssim r \lesssim 3.5)$ than with $N_{\mathrm{OB}}$. However, the lower end $(r<2.2)$ still includes most of the type Ia SNRs, without contamination by the other type. N103B is again the only outlier at $r=6.2$; at 
P. Maggi et al.: The population of X-ray supernova remnants in the Large Magellanic Cloud
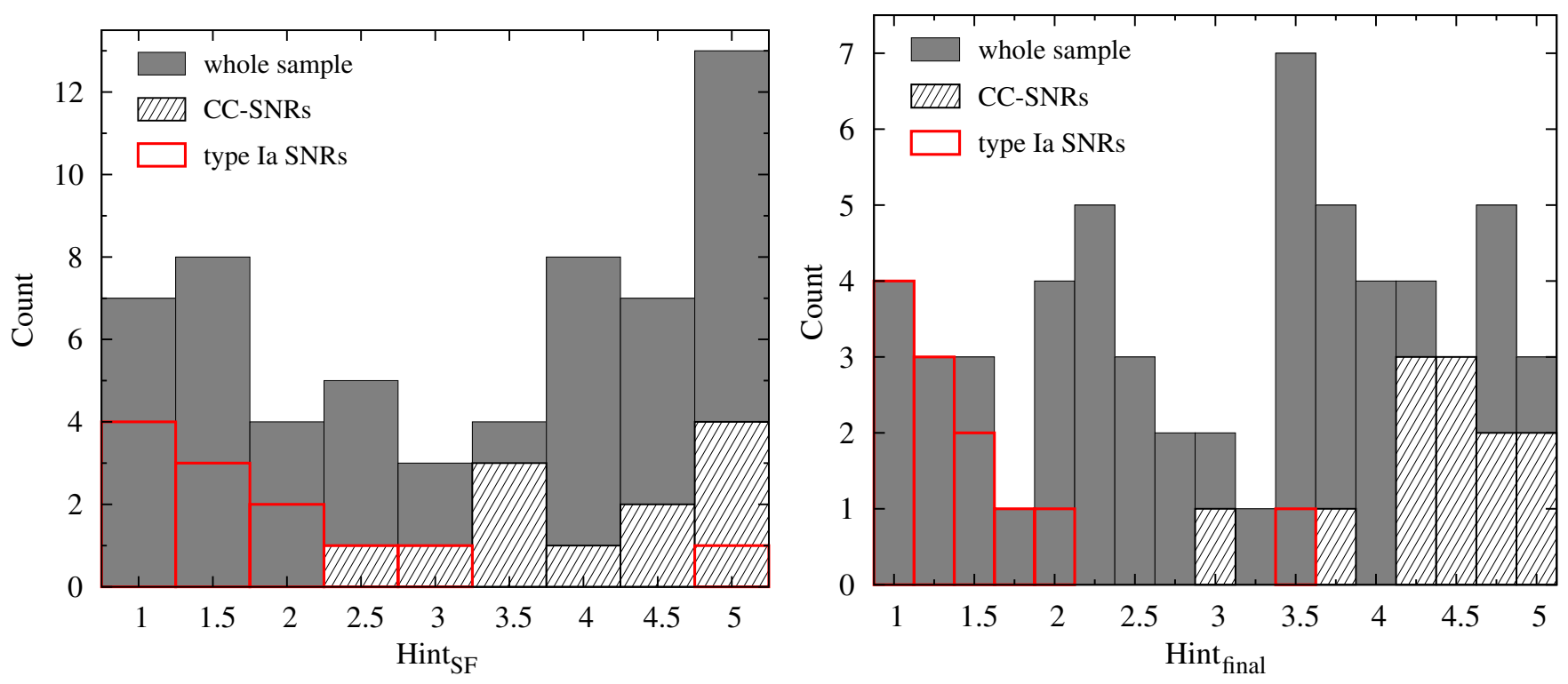

Fig. 10. Left: count distribution of the LMC SNRs as function of "Hint-SF", combining $N_{\mathrm{OB}}$ and $r$. Right: count distribution of the LMC SNRs as function of "Hint-final", combining spectral and SFH information (see text for details). Hatching and colours as in Fig. 9.

$r>3.4$ only CC-SNRs are found. In view of this observed distribution, the ratio $r=N_{\mathrm{CC}} / N_{\mathrm{Ia}}$ is still a useful tool to assign a type to SNRs using the observed local SFH, and should be valid in a comparative and statistical sense. The "Hints-SFH" attributed to the sample based on $r$ are listed in Table 6. $r$ and $N_{\mathrm{OB}}$ are also displayed as a scatter plot for secured Ia and CC SNRs (right panel of Fig. 9). There, the regions corresponding to different "Hints" are marked.

Caveat on the complementarity of $N_{\mathrm{OB}}$ and $r$ : it is clear that the two metrics are connected. Both are based on the MCPS catalogue; the early-type stars detected in a cell drive the fitting of the most recent time bins in the SFH reconstruction of Harris \& Zaritsky (2009). However, the $r$-value of a cell can be moderate even though $N_{\mathrm{OB}}$ is high, as evident from the scatter along the horizontal axis in Fig. 9 (right panel). That is because $r$ is a relative measure of the recent SFR compared to that at earlier epochs, while $N_{\mathrm{OB}}$ gives a measure of the absolute strength of the recent star formation. In the (high $N_{\mathrm{OB}}$ - moderate $r$ ) case, there are many available progenitors of both $\mathrm{CC}$ - and type Ia SN; these are typically cases where the classification is inconclusive.

Results for the whole sample: the count distributions for all LMC SNRs in the $N_{\mathrm{OB}}$ and $r$ spaces are shown in Fig. 9 (grey histograms, left and middle panels), and as a scatter plot in the right panel. They are similar, with larger numbers, to the distributions of the secured-type SNRs. About twenty remnants are in regions with a low number of early-type stars $\left(N_{\mathrm{OB}}<15\right)$ and not dominated by recent SF $(r \lesssim 2)$. There is a peak at $r \sim 6$ with a dozen remnants. Those are SNRs in star-forming regions which are widely spread across the LMC. They are often associated with giant H II complexes (e.g. LHA-120 N4, N11, N44). The objects with extreme values for $r(z 8)$ also have the largest $N_{\mathrm{OB}}$. Those are located in the two most intensively starforming regions of the LMC: 30 Doradus, and the rim of the supergiant shell LMC 4 (which embeds the "Constellation III" region, Harris \& Zaritsky 2008, 2009).
To combine the two "Hints" into one, we computed the arithmetic mean of Hint-CMD and Hint-SFH. The resulting "starformation Hint" (Hint-SF) again ranges from 1 to 5. Its distribution for the whole sample and the secured-type SNRs is shown in Fig. 10. There are 19 remnants with Hint-SF $\leq 2$; they most likely all result from a type Ia SN. We call this sample "likelyIa". Likewise, the 28 objects above Hint-SF $\geq 4$ are probably most of the CC-SNR population. They form the "likely-CC" sample.

The single type Ia SNR contaminating the sample (N103B) allows estimating a false-positive rate of 5\%-10\%. The falsepositive rate of the "likely-Ia" sample is probably lower: the massive stars formed at (roughly) the same time as the progenitor of a CC-SN can hardly be missed by photometric survey, because they would form the bright end of the population.

There are 12 SNRs in between $2.5 \leq$ Hint $_{\mathrm{SF}} \leq 3.5$, for which the local stellar environment cannot be used to decisively type the origin; they form the "SFH-untyped" sample. Interestingly, two and five of these remnants can be classified from other indicators as type Ia (the iron-rich MCSNR J0508-6830 and DEM L71) or CC-SNRs (e.g. the oxygen-rich N132D or MCSNR J0453-6829, which hosts a PWN), respectively.

Including the spectral results for typing purpose The spectral analysis of Sect. 5 revealed the presence of ejecta-enhanced plasma in almost half of the sample (Tables 3 and E.2). One should take advantage of this for the typing of the remnant, in combination with the SFH-based method we just presented. We assign another number, "Hint-spec", which depends on the high- or low-abundance flags of each SNR (Sect. 5.5). The numbers range from 1 (strongly favouring a type Ia origin) if "low $\mathrm{X} / \mathrm{Fe}$ " flags are raised (i.e. the SNR is iron-rich), to 5 (strongly favouring a $\mathrm{CC}$ origin) when "high $\mathrm{X} / \mathrm{Fe}$ " flags are raised (i.e. $\mathrm{CC}$ nucleosynthesis pattern is detected).

The choice of "Hint-spec" is given in Table 7. Note that a bigger impact is given to the low or high $\mathrm{O} / \mathrm{Fe}$ ratio, as these elements are the most abundant. Therefore, this ratio is easier to fit, more reliable, and sometimes the only one available. A value of 5 is also attributed to remnants where a pulsar/PWN is 
Table 7. "Hint-spec" attributed to SNRs as function of spectral results.

\begin{tabular}{|c|c|}
\hline Hint-spec & Criteria \\
\hline 1 & at least three "low $\mathrm{X} / \mathrm{Fe}$ " flags, no "high $\mathrm{X} / \mathrm{Fe}$ " flag \\
\hline 1.5 & two "low X/Fe" flags or low $\mathrm{O} / \mathrm{Fe}$, no "high $\mathrm{X} / \mathrm{Fe}$ " flag \\
\hline 2 & one "low $\mathrm{X} / \mathrm{Fe}$ " flag (except $\mathrm{O} / \mathrm{Fe}$ ), no "high $\mathrm{X} / \mathrm{Fe}$ " flag \\
\hline 2.5 & low $\mathrm{Si} / \mathrm{Fe}$, no "high $\mathrm{X} / \mathrm{Fe}$ " flag \\
\hline 3 & ISM abundances, unfitted abundances, or no XMM-Newton data \\
\hline 3.5 & high $\mathrm{Si} / \mathrm{Fe}$, no "low X/Fe" flag \\
\hline 4 & one "high X/Fe" flag (except $\mathrm{O} / \mathrm{Fe}$ ), no "low X/Fe" flag \\
\hline 4.5 & two "high $\mathrm{X} / \mathrm{Fe}$ " flags or high $\mathrm{O} / \mathrm{Fe}$, no "low $\mathrm{X} / \mathrm{Fe}$ " flag \\
\hline 5 & at least three "high $\mathrm{X} / \mathrm{Fe}$ " flags, no "low X/Fe" flag; pulsar/PWN detected \\
\hline
\end{tabular}

detected in the remnant. The values of "Hint-spec" for each SNR are given in Table C.1. We combined "Hint-SF" and "Hint-spec" by taking their arithmetic mean. The distribution of the resulting "Hint-final" is shown in Fig. 10 (right panel). The contamination (i.e. misclassification of N103B) is slightly alleviated, while a better separation of the "secured-type" SNRs is evident. There are 23 SNRs with "Hint-final" $\leq 2.5$ which are likely of type Ia, and 31 SNRs where "Hint-final" $\geq 3.5$ which are likely attributed to CC, although N103B (Hint-final = 3.5) is still contaminating the sample. There are five sources with inconclusive "Hint-final", including one secured-CC (N23).

\subsection{Ratio of CC to type la SNe and implications}

The observed number of SNRs of both types provides a measurement of the ratio of $\mathrm{CC}$ to type Ia SNe that exploded in the LMC over the last few $10^{4} \mathrm{yr}$, i.e. very close to the current ratio of CC/Ia SN rates. Based on the "star formation Hint", the numbers of SNRs in the "likely Ia" and "likely CC" samples translate in $N_{\mathrm{CC}} / N_{\mathrm{Ia}}=1.47(28 / 19)$. Assuming all "SFH-untyped" SNRs which do not have a secured type are of type Ia then the ratio $N_{\text {CC }} / N_{\text {Ia }}$ is 1.27 (33/26). Conversely, if the "SFH-untyped" are all $\mathrm{CC}$, the ratio is 1.81 (38/21). Even correcting for N103B, $N_{\text {CC }} / N_{\text {Ia }}$ is conservatively in the range 1.2 to 1.8 .

Including the spectral results (detection of ejecta in X-rays), we have a ratio $N_{\mathrm{CC}} / N_{\text {Ia }}=1.35(31 / 23)$. Correcting for N103B and N23 (with wrong and uncertain classifications), the ratio CC:Ia based on SFH and X-ray spectroscopy is between 1.11 $(31 / 28)$ and $1.46(35 / 24)$, depending on the type assigned to the remaining four objects. This range is compatible with that derived from the "SFH-typing" alone, albeit narrower because of the greater amount of information included in the calculation.

This ratio can be compared to two kinds of measurements: first, to the observed ratio of current rates, obtained from $\mathrm{SNe}$ search. For instance, Li et al. (2011) measured a ratio of about $3: 1$ in a volume-limited sample. Second, to the ratio $N_{\mathrm{CC}} / N_{\mathrm{Ia}}$ derived from intracluster medium (ICM) abundances. Galaxy clusters retain all the metals produced by SNe. The X-ray spectrum of the ICM reveals the elemental abundances, which are used to constrain the time-integrated numbers of $\mathrm{CC}$ and Ia SNe. From Suzaku observations of a small sample of clusters and groups of galaxies, Sato et al. (2007) estimated $N_{\mathrm{CC}} / N_{\mathrm{Ia}} \sim 3.5$ (ranging between 2 and 4, depending on the type Ia SN model assumeds). With XMM-Newton and a larger cluster sample, de Plaa et al. (2007) measured a $N_{\mathrm{CC}} / N_{\text {Ia }}$ between 1.7 and 3.5, again depending on the adopted SN Ia models. However, none of the explored $\mathrm{SN}$ models could reproduce the $\mathrm{Ar} / \mathrm{Ca}$ ratio. Lovisari et al. (2011) derived $N_{\text {CC }} / N_{\text {Ia }} \sim 1.5-3$. Therefore, the current ratio of $\mathrm{CC} / \mathrm{Ia} \mathrm{SNe}$ in the $\mathrm{LMC}$ is significantly lower than that measured in local SN surveys and in galaxy clusters.

One possible caveat could be that we are missing CC-SNRs. For instance, SNe exploding in superbubbles (SBs, see e.g. Chu 2008, for a review) will not be directly recognised as SNRs. Wang \& Helfand (1991) and Dunne et al. (2001) found a dozen LMC SBs with an X-ray luminosity, measured with Einstein and ROSAT, brighter than theoretically expected for a windblown bubble, and possibly energised by interior SNRs. The limited spatial resolution of the instruments used may result in distinct SNRs to have been overlooked and the X-ray emission of the SB overestimated (e.g. MCSNR J0523-6753, near the H II region/SB N44 in Wang \& Helfand 1991, see also Jaskot et al. 2011). With a dozen extra CC SNRs, the ratio $N_{\mathrm{CC}} / N_{\mathrm{Ia}}$ is pushed to $\sim 1.5-2$. However, the number of type Ia SNRs currently known in the LMC is also expected to be below the actual number (see Sect. 7 for a discussion on sample completeness). Therefore, it is unlikely that the ratio $N_{\mathrm{CC}} / N_{\mathrm{Ia}}$ is significantly underestimated. Furthermore, the abundance pattern of the LMC, with its low $[\alpha / \mathrm{Fe}]$ (Sect. 5.6), lends support to such a low $N_{\mathrm{CC}} / N_{\text {Ia }}$. This should be lower than the value of $N_{\mathrm{CC}} / N_{\mathrm{Ia}} \sim 4-5$ estimated by Tsujimoto et al. (1995) using metallicity alone.

The low $N_{\mathrm{CC}} / N_{\text {Ia }}$ ratio measured in the LMC therefore has to be a consequence of the different SFH of the Cloud compared to that in other nearby galaxies or galaxy clusters. The local SN rates depend on the summed $\mathrm{SFH}$ of galaxies included in the SN surveys. The higher ratio measured by e.g. Li et al. (2011) simply indicates that many star-forming galaxies are included in the local volume explored. The SFHs of galaxy clusters are relatively simple, with short episodes of star-formation at a redshift of $z \sim 3$ (Eisenhardt et al. 2008), so that the integrated numbers of type Ia and CC SNe inferred from X-ray observations correspond to the fractions of stars formed that end their lives as $\mathrm{SN}$ of either type.

In the LMC, star formation occurred during several episodes. In addition to many regions with recent or ongoing star formation where, unsurprisingly, the CC-SNRs are found (see Sect. 8), the LMC had enhanced star formation episodes $100 \mathrm{Myr}$, $500 \mathrm{Myr}$, and 2 Gyr ago as well (Harris \& Zaritsky 2009). The SN Ia DTD follows fairly well a $t^{-1}$ power law for delays $t>1 \mathrm{Gyr}$, and appear to keep increasing below $1 \mathrm{Gyr}$ (for a review of SN Ia DTD measurements, see Maoz \& Mannucci 2012). The majority of type Ia SNe explode within 2 Gyr after star-forming episodes. We are therefore coincidentally observing the LMC at a time when the rate of type Ia SN from the stellar populations formed $500 \mathrm{Myr}$ to $2 \mathrm{Gyr}$ ago is high. Integrated over an SNR lifetime (a few $10^{4} \mathrm{yr}$ ), it results in the relatively large number of type Ia SNRs. It is not possible to use $N_{\text {CC }} / N_{\text {Ia }}$ to estimate $\eta$, the fraction of stars that eventually explode as Ia SNe (Maoz 2008), because of the complex SFH of 
the LMC: stars exploding now (as either SN types) were created at different epochs. Furthermore, $\eta$ is also dependent on the initial mass function (IMF), over which one has little freedom, since the SFH-reconstruction already assumes a particular form (the Salpeter IMF).

Currently, the only other galaxy with which to compare the $N_{\text {CC }} / N_{\text {Ia }}$ of the LMC is the SMC. In our own Milky Way, there remain too many untyped SNRs. More problematic are the distance uncertainties and line-of-sight confusion that prevent associating remnants to regions of star formation (e.g. spiral arms). In the Local Group (M31, M33) and beyond (e.g. M83, Dopita et al. 2010), the problem is again the lack of secured typing methods, and generally the absence of spatially resolved SFH. The situation is likely to improve in the near future with more sensitive X-ray observatories (e.g. Athena), and large observing programmes of M31 and M33 with Hubble which allow SFH maps to be built (so far, this was done in the few archival field available, Jennings et al. 2012, 2014). The SMC is the only obvious target remaining where a similar study can be currently performed, although the smaller sample of SNRs and inclination of the galaxy (and corresponding line-of-sight confusion) might complicate direct comparisons to the LMC.

\section{X-ray luminosity function of SNRs in the Local Group}

X-ray luminosity functions (XLFs) are valuable tools for the study of X-ray sources and comparisons between populations. The XMM-Newton dataset is ideally suited to derive the XLF of LMC SNRs. Out of the 59 objects in the sample, XMM-Newton covered 51 of them, to which we fitted spectral models (Sect. 5). For all these, the X-ray fluxes in various bands are obtained from the best-fit models (Tables E.1 and E.2) with the XSPEC command flux. The final results are presented in the "broad" band, from $0.3 \mathrm{keV}$ to $8 \mathrm{keV}$ (the effect of including the high-energy part is very minor and discussed below).

Three SNRs have been covered with Chandra but not XMM-Newton: for MCSNR J0454-6713 (N9), we used the spectral results of Seward et al. (2006) to measure the flux. MCSNR J0459-7008 (N186D) was covered in the Chandra observation of the SB DEM L50. Jaskot et al. (2011) published the results from these data. We used their best-fit NEI model for the SNR emission, which is spatially resolved from the SB, to derive the X-ray flux. Finally for MCSNR J0550-6823, we used the spectral parameters given in the entry of the Chandra SNR catalogue $^{17}$.

Two SNRs have neither XMM-Newton nor Chandra observations available, but were covered with ROSAT. Williams et al. (1999b) present a spectral analysis of MCSNR J0455-6839 (in N86). We obtained the X-ray flux of the SNR using their best-fit model. MCSNR J0448-6700 corresponds to the ROSAT PSPC source [HP99] 460, with a count rate of $1.41 \times 10^{-2} \mathrm{cts} \mathrm{s}^{-1}$ (Haberl \& Pietsch 1999). With the multi-mission count rate simulator WebPIMMS ${ }^{18}$, we calculated the flux in several energy bands for various temperatures of an APEC model, assuming a total absorbing column $N_{\mathrm{H}}=7 \times 10^{20} \mathrm{~cm}^{-2}$ towards the source and a mean abundance of 0.4 solar. The observed hardness ratios could be reproduced for $k T=0.97 \mathrm{keV}$. These

\footnotetext{
${ }^{17}$ Maintained by Fred Seward: http://hea-www.cfa.harvard. edu/ChandraSNR/index . html

18 http://heasarc.gsfc.nasa.gov/cgi-bin/Tools/w3pimms/ w3pimms.pl
}

spectral parameters and normalisation can be converted into fluxes in the same bands as used for the rest of the sample.

In total, 56 objects have well-defined X-ray fluxes and make it into the XLF. The adopted values are listed in Table C.1. Only three SNRs have no X-ray data available. The cumulative XLF is shown in Fig. 11. The sample spans almost four orders of magnitude in luminosity, from the brightest (N132D) at $L_{\mathrm{X}}=3.15 \times 10^{37} \mathrm{erg} \mathrm{s}^{-1}$ down to $\sim 7 \times 10^{33} \mathrm{erg} \mathrm{s}^{-1}$. The $L_{\mathrm{X}}$ used is the observed value, uncorrected for (LMC) absorption, because the fitted column densities can be quite uncertain, in particular in the faint end. We checked the $N_{\mathrm{H} \text { LMC-corrected XLF }}$ and found no significant variations in its shape compared to that of Fig. 11, except the shift to higher $L_{\mathrm{X}}$.

SNR XLF from other Local Group galaxies: the LMC XLF can be best compared to other Local Group galaxies such as M31, M33, and the SMC. Sasaki et al. (2012) studied M31 SNRs and candidates identified in the XMM-Newton Large Programme survey of the Andromeda galaxy (Stiele et al. 2011). They converted EPIC count rates into $0.35 \mathrm{keV}-2 \mathrm{keV}$ luminosities assuming a thermal (APEC) spectrum with $k T=0.2 \mathrm{keV}, N_{\mathrm{H} \text { M31 }}=$ $10^{21} \mathrm{~cm}^{-2}$, and $N_{\mathrm{H} \mathrm{MW}}=0.7 \times 10^{21} \mathrm{~cm}^{-2}$. The quoted values, however, are corrected for $N_{\mathrm{H}} \mathrm{MW}$, while for the LMC we give the observed luminosities. For consistency with the LMC XLF, we re-included $N_{\mathrm{H} \text { MW }}=0.7 \times 10^{21} \mathrm{~cm}^{-2}$ in the results of Sasaki et al. (2012) and converted the luminosity in the $0.3 \mathrm{keV}-8 \mathrm{keV}$ by scaling their $L_{X}$ by 0.577 (a factor derived from simulating their assumed spectrum with and without $N_{H}$ Mw). Note that the effect of the foreground absorption should be very minor, since

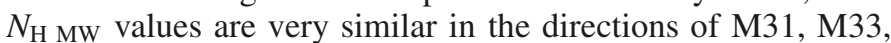
and the LMC $\left(5-7 \times 10^{21} \mathrm{~cm}^{-2}\right) .26$ objects were confirmed SNRs in Sasaki et al. (2012), and another 20 were candidate SNRs.

Long et al. (2010) present a large catalogue of 82 confirmed SNRs in M33, based on the Chandra ACIS survey of M33 (ChASeM33, Tüllmann et al. 2011). They give $L_{X}$ in the $0.35 \mathrm{keV}-2 \mathrm{keV}$ band, converted from ACIS count rates, assuming a thermal plasma at 0.5 solar, $k T=0.6 \mathrm{keV}$, and total $N_{\mathrm{H}}=10^{21} \mathrm{~cm}^{-2}$ (i.e. the spectrum found for the brightest source). We obtained the corresponding $0.3 \mathrm{keV}-8 \mathrm{keV}$ luminosity by scaling up the values of Long et al. (2010) by $4 \%$.

Recently, Williams et al. (2015) published the results of a deep XMM-Newton survey of M33 with a larger coverage than ChASeM33, up to the $D_{25}$ isophote of the galaxy. They recovered most of the SNRs of Long et al. (2010), except in the central region where source confusion is an issue for XMM-Newton, and detected or confirmed eight new SNRs, including three sources far in the outskirts of M33. We converted the unabsorbed $0.2-4.5 \mathrm{keV}$ EPIC flux of the new inclusions of Williams et al. (2015), obtained assuming a power-law spectrum, to the 0.3-8 keV luminosity for the same spectrum as the SNRs of Long et al. (2010). The final, concatenated list of M33 SNRs thus comprises 90 objects.

Converting count rate to luminosity in different energy bands assuming a single temperature might affect the slope of the XLF. For instance, from a count rate in the $0.35 \mathrm{ke}-2 \mathrm{keV}$ band, the luminosity in the broad band is $25 \%$ higher with $k T=0.6 \mathrm{keV}$ than if it is $0.2 \mathrm{keV}$. The two studies have limited knowledge of the actual spectrum of each remnant, because the larger distances prohibit more complex spectral fits, and they have to assume a particular spectrum, regardless of luminosity. This is not the case in the LMC. We indeed found a trend for brighter remnants to have higher plasma temperatures (Sect.5.1). Quantitatively, 


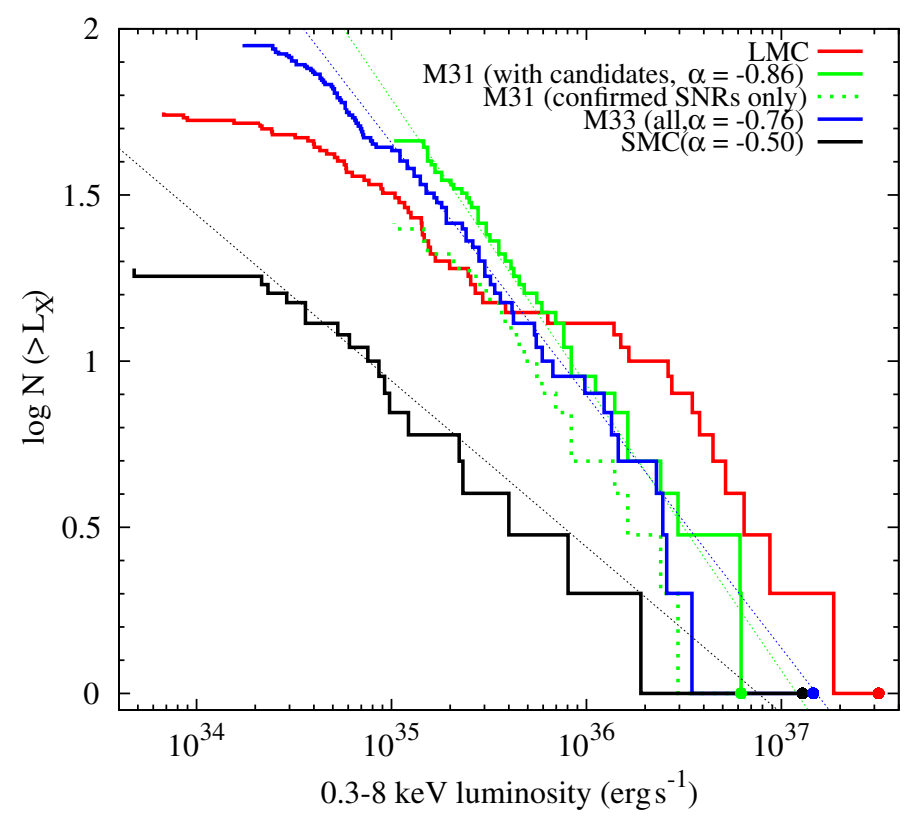

Fig. 11. Cumulative X-ray luminosity function of SNRs in Local Group galaxies. See text for details and references on how $L_{X}$ was measured for each sample. The brightest SNR in each galaxy is marked by a dot. The thin dotted lines are nonlinear least-square fits of a power law $\left(N\left(>L_{\mathrm{X}}\right) \propto L_{\mathrm{X}}{ }^{\alpha}\right)$. Slopes $\alpha$ are given in the legend. These fits are only used to characterise the slopes and illustrate the differences between galaxies; they do not represent a physical fit of the population.

the median temperatures are $k T=0.31 \mathrm{keV}$ for luminosities less than $10^{35} \mathrm{erg} \mathrm{s}^{-1}, 0.55 \mathrm{keV}$ between $10^{35} \mathrm{erg} \mathrm{s}^{-1}$ and $10^{36} \mathrm{erg} \mathrm{s}^{-1}$, and $0.8 \mathrm{keV}$ above $10^{36} \mathrm{erg} \mathrm{s}^{-1}$. The luminosities of M31 SNRs were given assuming $k T=0.2 \mathrm{keV}$; we scaled the $0.3 \mathrm{keV}-8 \mathrm{keV}$ luminosity up by $1.05,1.20$, and 1.35 for sources with $L_{\mathrm{X}}<10^{35}, 10^{35}<L_{\mathrm{X}}<10^{36}$, and $L_{\mathrm{X}}>10^{36} \mathrm{erg} \mathrm{s}^{-1}$, respectively. M33 SNRs were assumed to have a higher temperature $(0.6 \mathrm{keV})$, which means that the luminosity of objects below $\sim 10^{35} \mathrm{erg} \mathrm{s}^{-1}$ was overestimated by about $15 \%$, while for those above $10^{36} \mathrm{erg} \mathrm{s}^{-1}$ it was underestimated by $\sim 8 \%$. Correcting for this effect ensures a meaningful comparison between M31, M33, and the LMC.

The SMC SNR population is comparatively smaller. van der Heyden et al. (2004) presented an X-ray spectral analysis of all SNRs in the SMC known at that time. We used their best-fit models to measure the observed (i.e. absorbed) X-ray luminosity in the same $0.3 \mathrm{keV}-8 \mathrm{keV}$ band $^{19}$, except for IKT 16 . For this SNR we used results from Owen et al. (2011), which included more data from subsequent XMM-Newton observations. Three additional SNRs were covered with XMM-Newton; the results were published in Filipović et al. (2008), from which we borrowed the best-fit spectral models. The latter study also reported a new SNR, HFPK 334. For this one, we used the best-fit model from Crawford et al. (2014), which combined XMM-Newton and Chandra observations. Also included is the SNR XMMU J0056.5-7208 identified during the SMC survey (Haberl et al. 2012b; Sturm 2012). Finally, the Be/X-ray binary pulsar SXP 1062 was found to be associated to an SNR, of which it is most likely the progenitor (Hénault-Brunet et al. 2012). The thermal emission from the SNR was studied by Haberl et al. (2012c). This sample of 19 SMC SNRs is the most up to date. 19 The luminosity given in van der Heyden et al. (2004), Table 3, for
IKT $22(1 \mathrm{E} 0102-7219$, the brightest SMC SNR) was mistyped. Instead
of the $150 \times 10^{27} \mathrm{~W}$, it should read $1500 \times 10^{27} \mathrm{~W}\left(1.5 \times 10^{37} \mathrm{erg} \mathrm{s}^{-1}\right)$.
Comparative study of SNR XLFs: the cumulative XLFs of $\mathrm{M} 31$ and M33 in the $0.3 \mathrm{keV}-8 \mathrm{keV}$ band, corrected for the $k T-L_{\mathrm{X}}$ trend, are shown along that of the SMC and LMC in Fig. 11. In terms of depth, the LMC XLF dominates. There is a single SNR at $L_{X}<2 \times 10^{34} \mathrm{erg} \mathrm{s}^{-1}$ in M33 and in the SMC, but the bright interior pulsar in the SMC case (SXP 1062) makes the measurement of the thermal emission luminosity uncertain. In contrast, there are eight SNRs with $L_{X} \lesssim 2 \times 10^{34} \mathrm{erg} \mathrm{s}^{-1}$ in the LMC, of which seven were discovered or confirmed thanks to XMM-Newton observations.

In terms of number, the largest population so far is found in M33 (90 SNRs in X-rays), probably owing to the depth of the Chandra survey (using $100 \mathrm{ks}$ pointings) in the central $15^{\prime}$, the overlap with a deep XMM-Newton survey up to the $D_{25}$ isophote, and the favourable (face-on) orientation of M33. However, the population of M31 SNRs is larger than any other at $L_{\mathrm{X}} \lesssim 5 \times 10^{35} \mathrm{erg} \mathrm{s}^{-1}$ and is only limited by the depth of the survey $\left(\sim 10^{35} \mathrm{erg} \mathrm{s}^{-1}\right)$. The ratio of M31-to-M33 SNRs in the $10^{35}-10^{36} \mathrm{erg} \mathrm{s}^{-1}$ range is at most 1.5 , i.e. substantially smaller than the mass ratio of the galaxies (10-20, Corbelli 2003; Peñarrubia et al. 2014). This shows the effect of the higher (recent) SFR in M33 compared to M31 (0.45 $M_{\odot} \mathrm{yr}^{-1}$ vs. $0.27 M_{\odot} \mathrm{yr}^{-1}$, Verley et al. 2009; Tabatabaei \& Berkhuijsen 2010) leading to a larger production of CC SNRs in M33. In the same luminosity range, the number of LMC SNRs is comparable to that in M33. This is expected because the LMC is only slightly less massive than M33. Furthermore, the recent SFR of the LMC is high, 0.3-0.4 $M_{\odot} \mathrm{yr}^{-1}$ in the last $40 \mathrm{Myr}$ (Harris \& Zaritsky 2009). This conspires with the high current type Ia SN rate (Sect. 6.4) to build up the large population of SNRs in the LMC. Finally, the "feather-weight" SMC (about ten times less massive than the LMC, (Stanimirović et al. 2004; Harris \& Zaritsky 2006) has a smaller, yet decent population of remnants, likely owing to its recent star formation activity (0.08-0.3 $M_{\odot} \mathrm{yr}^{-1}$, Harris \& Zaritsky 2004).

In terms of shape, the XLF of M31 SNRs is the most uniform, following a power law $\left(N\left(>L_{\mathrm{X}}\right) \propto L_{\mathrm{X}}{ }^{\alpha}\right)$ with $\alpha=-0.86 \pm$ 0.04 down to $\sim 2 \times 10^{35} \mathrm{erg} \mathrm{s}^{-1}$. This holds with or without including the candidates, which means that most are indeed bonafide SNRs. The M33 remnants follow mostly the same distribution, with $\alpha=-0.76 \pm 0.05$. Towards the faint end, the M33 XLF flattens and diverges from the power law below $10^{35} \mathrm{erg} \mathrm{s}^{-1}$, indicating incompleteness. Long et al. (2010) concluded that no SNR brighter than $4 \times 10^{35}$ erg s$^{-1}$ was missed across the surveyed field. It is likely that they were over-conservative and that missing SNRs are only those which have luminosity below $10^{35} \mathrm{erg} \mathrm{s}^{-1}$. The combined ChASeM33 and XMM-Newton surveys cover the total extent of the galaxy (Plucinsky et al. 2008; Williams et al. 2015), so the missing SNRs are either too X-rayfaint (below the surveys' detection limits), or absent/undetected at radio and optical wavelengths, precluding their identifications as SNRs.

We performed Kolmogorov-Smirnov (KS) tests to compare the different populations. Using a bootstrapping method, we produced 1000 luminosity functions from the original data. We checked that similar results were obtained when increasing that number to $10^{6}$. Restricting the analysis to SNRs brighter than $3 \times 10^{35} \mathrm{erg} \mathrm{s}^{-1}$ to ensure completeness of the samples, we found that the XLFs of M31 and M33 SNRs follow the same distribution at the $3 \sigma$ confidence level. There was a marginal indication that the M33 distribution was steeper than that of M31 (Sasaki et al. 2012), but this difference essentially disappears once the $k T-L_{\mathrm{X}}$ trend is taken into account. 
In the SMC, although the population is limited to about 20 objects, the distribution is relatively uniform. The XLF is however flatter $(\alpha=-0.5 \pm 0.05)$, and KS tests confirm that the SMC population is different from those of M31 and M33. This might indicate that SMC remnants evolve faster (and fade earlier) than in M31 and M33, due to a lower ISM density. The lower metallicity in the SMC (about 0.2 solar, Russell \& Dopita 1992) may also participates in the lower luminosities of the SMC SNR, since the emissivity of hot plasmas is smaller for lower metallicities.

In contrast to the other galaxies, the luminosity function of SNRs in the LMC exhibits a complex behaviour and does not obey a smooth power-law distribution over most of the dynamical range. Regardless of the lower luminosity cut used, the KS tests reject the null hypothesis that LMC SNRs have the same XLF than those in M31, M33, or the SMC.

The most striking and robust result is the very prominent bright end of the LMC XLF. There are 13 SNRs with $L_{X}>$ $10^{36} \mathrm{erg} \mathrm{s}^{-1}$, more than in M31 and M33. Amongst these, there are two SNRs hosting bright pulsars/PWNe and a harder nonthermal spectrum. Even restricting the XLF to the soft band or excluding these two objects, the population of bright LMC SNRs is still above the other ones. This bright population is not a clearly distinct group. In particular, it is not made up of remnants from only one SN type. There are four type Ia SNRs and nine CC-SNRs, so the $N_{\mathrm{CC}} / N_{\text {Ia }}$ ratio is higher than overall (Sect. 6.4), but not exceedingly so. Higher luminosities are expected from SNRs interacting with denser ISM. We compared the average LMC H I column density (from the map of Kim et al. 2003) around the position of remnants in various luminosity bins, but no trend could be found. However, the line-of-sight integrated column density might not be a good indicator of the ISM density local to the remnant, considering that the SNR could be in front of or behind the regions where most of the neutral hydrogen is (see Sect. 8).

A possible explanation for the population of bright SNRs in the LMC stems from its lower metallicity. Massive stars lose a considerable amount of mass in the form of winds (e.g. Kudritzki \& Puls 2000). The stellar winds blow low-density cavities, bordered by dense shells, around the stars that eventually explode as (core-collapse) SNe. The interaction of the SN shocks with the modified CSM results in a different evolution compared to that in a constant-density ISM. Dwarkadas (2005, 2007, and references therein) explored the evolution of remnants in wind-blown cavities. It was shown that it critically depends on one parameter (coined $\Lambda$ ), the ratio of the mass of the dense shell to that of the ejected material. For low values $(\Lambda<1)$ the X-ray luminosity increases sharply when the shock reaches the dense shell early on $\left(t<10^{3} \mathrm{yr}\right)$. If instead the shell is more massive compared to the ejecta material, the shock propagates in the very low density of the (much larger) bubble, producing less X-ray emission. The increase of X-ray luminosity upon impact (after a few thousand years) is also smaller than in the low- $\Lambda$ case (Dwarkadas 2005, his Figs. 7 and 12). The properties of the cavities around massive stars are determined by the mass loss rate $\dot{M}$ during their various evolutionary stages. This in turn is affected by the elemental abundance (i.e. metallicity), because the main driving mechanism of stellar winds is the transfer of momentum from photons to the star atmospheric gas by line interactions $^{20}$ (Kudritzki \& Puls 2000; Vink et al. 2001). By measuring mass-loss rates of early-type stars in the Galaxy, LMC, and

\footnotetext{
20 The product abundance $\times$ ionisation fraction $\times$ number of available lines for metals is comparable to that of hydrogen and helium.
}

SMC, Mokiem et al. (2007) could quantify the dependence of $\dot{M}$ on the metallicity as $\dot{M} \propto Z^{0.83}$. It is therefore expected that in lower metallicity environment (e.g. LMC) massive stars explode in wind-blown cavities with lower $\Lambda$, and are more likely to produce young remnants that are brighter in X-rays. Since the SMC has the lowest metallicity of our sample of galaxies ( 0.2 solar), one would also expect an excess of bright sources. However, the SMC does not host SNRs as young (less than a few thousand years) as the LMC (van der Heyden et al. 2004), and it is likely that the smaller wind-blown bubbles do not affect the X-ray luminosities of more evolved remnants. Furthermore, the small number of SNRss in the SMC hinders conclusions regarding a possible excess of bright sources.

Finally, there are also four type Ia SNRs amongst the bright end of the population, to which the explanation discussed above does not apply. If we exclude, these however, there is still an excess. Because they are prominently young (three are less than a thousand years old), it appears that the high current type Ia $\mathrm{SN}$ rate in the LMC (Sect. 6.4) will also contribute to a larger population of bright remnants.

Between $\sim 1 \times 10^{35} \mathrm{erg} \mathrm{s}^{-1}$ and $5 \times 10^{35} \mathrm{erg} \mathrm{s}^{-1}$, where many SNRs reside (a third of the sample), the LMC XLF is comparable in shape to the M31 and M33 XLFs, with a powerlaw distribution (consistent with $\alpha$ between -1 and -0.8 ), and in number to M33 (M31 begins to have more sources below $\sim 8 \times 10^{35} \mathrm{erg} \mathrm{s}^{-1}$ ). Towards the fainter end, the LMC XLF is again remarkable via its significant flattening. It is unlikely that this represents an overall flatter distribution (at least not as strongly as in the SMC), because it would imply that a lot of SNRs with $L_{X} \sim(5-8) \times 10^{35} \mathrm{erg} \mathrm{s}^{-1}$ (thus easy to identify) have been missed. It is more plausible that the flattening of the XLF is almost exclusively due to incompleteness. The majority of the remnants at $L_{\mathrm{X}}<8 \times 10^{34} \mathrm{erg} \mathrm{s}^{-1}(15 / 22)$ were identified/confirmed thanks to (pointed or serendipitous) XMM-Newton observations. Though many were already detected with ROSAT, the combination of the large effective area and resolution of XMM-Newton is usually needed to confirm the extent and thermal emission of candidates. Even with the VLP survey, the area of the LMC covered by XMM-Newton is less than 20 square degrees, i.e. about a third of the whole galaxy. In particular, X-ray coverage to the south-west of the LMC Bar is sparse (see Fig. 1). Extending the covered fraction warrants to find the missing remnants. The M31 survey with XMM-Newton exemplifies how a full coverage results in a high completeness: the M31 SNR XLF is uniform down to the sensitivity limit of the survey, which fully covers the $D_{25}$ ellipse of M31 (Sasaki et al. 2012). In the LMC, the situation could easily be improved with more X-ray observations. We briefly discuss possible strategies in Sect. 9.

\section{3D spatial distribution}

\subsection{Comparison with other wavelengths}

The positions of SNRs in the LMC are plotted on the H I column density map of Kim et al. (2003), showing the LMC gas disc (Fig. 12). The population exhibits correlations with neutral hydrogen structures. The most striking example is the many SNRs (a dozen) around the supergiant shell (SGS) LMC 4 (Meaburn 1980, SGS 11 in the notation of Kim et al. 1999). SGSs are formed by the combined action of multiple generations of massive star formation. Their expansions shock and sweep up the ISM, which can trigger further star formation along the SGS rims (Elmegreen 1998, and references therein). The impact of 


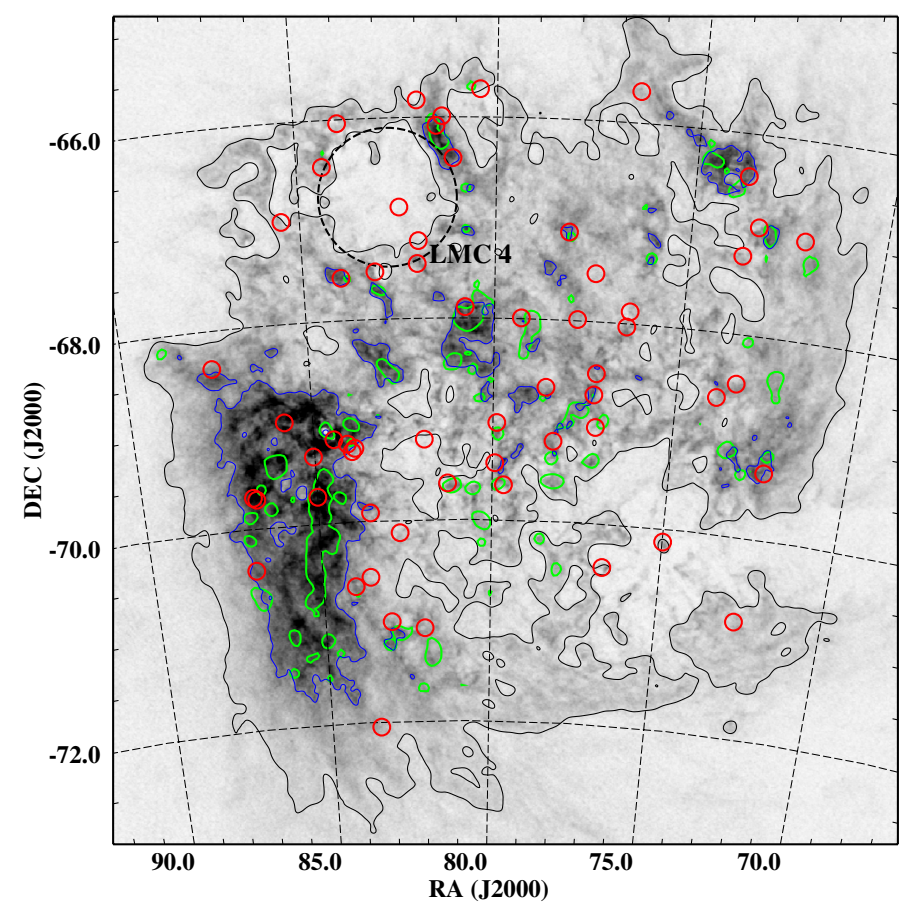

Fig. 12. Positions of LMC SNRs (red circles) on the H I column density map of Kim et al. (2003), displayed on a linear scale ranging from 0 to $6 \times 10^{21} \mathrm{~cm}^{-2}$. Black and blue contours indicate levels of 1 and $3 \times 10^{21} \mathrm{~cm}^{-2}$, respectively. The green contours are the $3 \sigma$ level $\left(1.2 \mathrm{~K} \mathrm{~km} \mathrm{~s}^{-1}\right)$ of the velocity-integrated map of ${ }^{12} \mathrm{CO}(J=1-0)$ from the NANTEN survey (Fukui et al. 2008). The position of the SGS LMC 4 is marked with a dashed black circle.

SGSs on star formation, particularly in the LMC, was demonstrated by Yamaguchi et al. (2001a,b). They found that the concentration of molecular clouds and young star clusters is enhanced by a factor of 1.5-2 near the SGS rims, and most of these clusters are on the side of the molecular clouds facing the interior of the SGSs. Book et al. (2009) added massive young stellar objects and $\mathrm{H}$ II regions/OB associations to the list of tracers of recent star formation that are well correlated with the shell peripheries.

Because (core-collapse) SNRs are themselves very good indicators of recent star formation, the distribution of many SNRs around the edge of LMC 4 is a further sign of the important role played by SGSs in triggering star formation. In turn, this could be used to look for new SNRs: the high number of remnants around LMC 4 is explained in part by the large size of the SGS $(\sim 1.2 \mathrm{kpc})$, but also by the good X-ray coverage (only two out of twelve SNRs around LMC 4 were not observed with XMM-Newton). Exploring SGSs less well studied, e.g. in the west and south-west regions of the LMC, is promising, as we discuss in Sect. 9.

Another prominent $\mathrm{HI}$ feature is the density enhancement in the east that extends southwards into "arms B and E" (see Fig. 1 of Staveley-Smith et al. 2003), which are interpreted as tidal features. Most of the SNRs in the south-east of the LMC are associated to the 30 Doradus complex (which itself might be a manifestation of tidal shear). Only a handful of sources are known in the regions of the $\mathrm{B}$ and $\mathrm{E}$ arms (and a single SNR is confirmed south of a declination of $-71^{\circ}$, Bozzetto et al. 2013). The southern region of the LMC is poorly studied in X-rays, preventing conclusions regarding the dearth of SNRs observed there. However, it could be an interesting target for future studies (Sect. 9).

In Fig. 13, we show the position of SNRs relative to $\mathrm{H} \alpha$ (left, MCELS data), and to a red continuum image from the SHASSA survey (Gaustad et al. 2001). The association of many CC-SNRs with large $\mathrm{H}$ II regions, which trace regions of active star formation, is evident: out of 31 secured or "likely" CC SNRs, 25 correlate with strong $\mathrm{H} \alpha$ emission, three with moderate $\mathrm{H} \alpha$ emission, and only three are not associated to large optical nebulosities. In contrast, many SNRs are not associated to $\mathrm{H} \alpha$ emission, e.g. in the Bar, or south-east and north-west of it. These are the type Ia SNRs: 22 out of 24 secured or "likely" Ia SNRs have no coincident $\mathrm{H} \alpha$ emission (except the remnant's emission itself). Only $\mathrm{N} 103 \mathrm{~B}$ is spatially associated to strong $\mathrm{H} \alpha$ emission, although it is plausibly a projection effect, with the SNR on the far side of the LMC (as suggested by its large " $N_{\mathrm{H}}$ fraction", see Sect. 8.2). This would resolve the long-standing issue of the association of a type Ia SNR with a region of intense star formation (Chu \& Kennicutt 1988; Badenes et al. 2009). The type Ia SNRs are in regions of relatively high stellar density (e.g. the Bar, as traced in the red continuum image) but are also present in more isolated, less active regions, where intermediate- and old-age stellar populations dominate.

\subsection{Adding the third dimension}

So far, we discussed the 2D distribution of SNRs, projected on the sky. It is possible to gain a rudimentary sense of depth, by comparing the absorbing column density derived from $\mathrm{X}$-ray observations (hereafter $N_{\mathrm{H}}^{\mathrm{X}}$ ), to the line-of-sight integrated H I column density, derived from $21 \mathrm{~cm}$ observations (hereafter $N_{\mathrm{H}}^{21 \mathrm{~cm}}$ ). We recall that $N_{\mathrm{H}}^{\mathrm{X}}$ is an equivalent neutral hydrogen column density assuming a given chemical composition ${ }^{21}$. The ratio $N_{\mathrm{H}}^{\mathrm{X}} / N_{\mathrm{H}}^{21 \mathrm{~cm}}$ (hereafter " $N_{\mathrm{H}}$ fraction") is a measurement of how deep an SNR is with respect to the H I structure. Interpreting the $N_{\mathrm{H}}$ fraction is made easier by the favourable orientation of the LMC. Neutral hydrogen is mainly distributed in a nearly circular disc at a moderate inclination angle ${ }^{22}$, with a thickness of $\sim 360$ pc (Kim et al. 1999). Small $N_{\mathrm{H}}$ fractions $(\lesssim 0.3$, e.g. when $N_{\mathrm{H}}^{\mathrm{X}}$ is consistent with zero) indicate that the SNR is well in front of the disc; intermediate values $(0.3$ to 0.8$)$ are expected from sources within the disc; high fractions $(0.8-1.2$; a value of 1.23 is expected when including contributions of neutral and singly ionised helium, Arabadjis \& Bregman 1999) are associated to remnants on the far side, or behind, the disc. Values significantly above 1.2 are discussed below.

$N_{\mathrm{H}}^{\mathrm{X}}$ is taken from the spectral results of Sect. 5. For the $1 \mathrm{~T} / 2 \mathrm{~T}$ sample, the adopted value is simply that in Table E.1. Only two $2 \mathrm{~T}$ remnants have two different absorption components: for MCSNR J0517-6759 we used the higher value. For MCSNR J0535-6602 (N63A), the highly absorbed component is ejecta-rich and has a lower EM; we therefore adopted the (lower) $N_{\mathrm{H}}$ of the ISM component, which is more representative. For the brightest SNRs, we adopted the best-fit values given in Table E. 2 and in Sect. 5.3 (for SNR 1987A). For three SNRs with

${ }^{21} \mathrm{X}$-rays are absorbed not only by $\mathrm{H} \mathrm{I}$, but also by molecular hydrogen, helium, and metals (Wilms et al. 2000).

${ }^{22}$ Measurements of the orientation of the disc, i.e. inclination $i$ (with $0{ }^{\circ}$ defined as face-on) and position angle of line of nodes $\Theta$ (the intersection of the disc and sky planes, measured eastwards of north), are widely scattered but are in the range $25^{\circ}<i<40^{\circ}$ and $120^{\circ}<\Theta<155^{\circ}$ (Westerlund 1997; Subramanian \& Subramaniam 2013; van der Marel \& Kallivayalil 2014). 

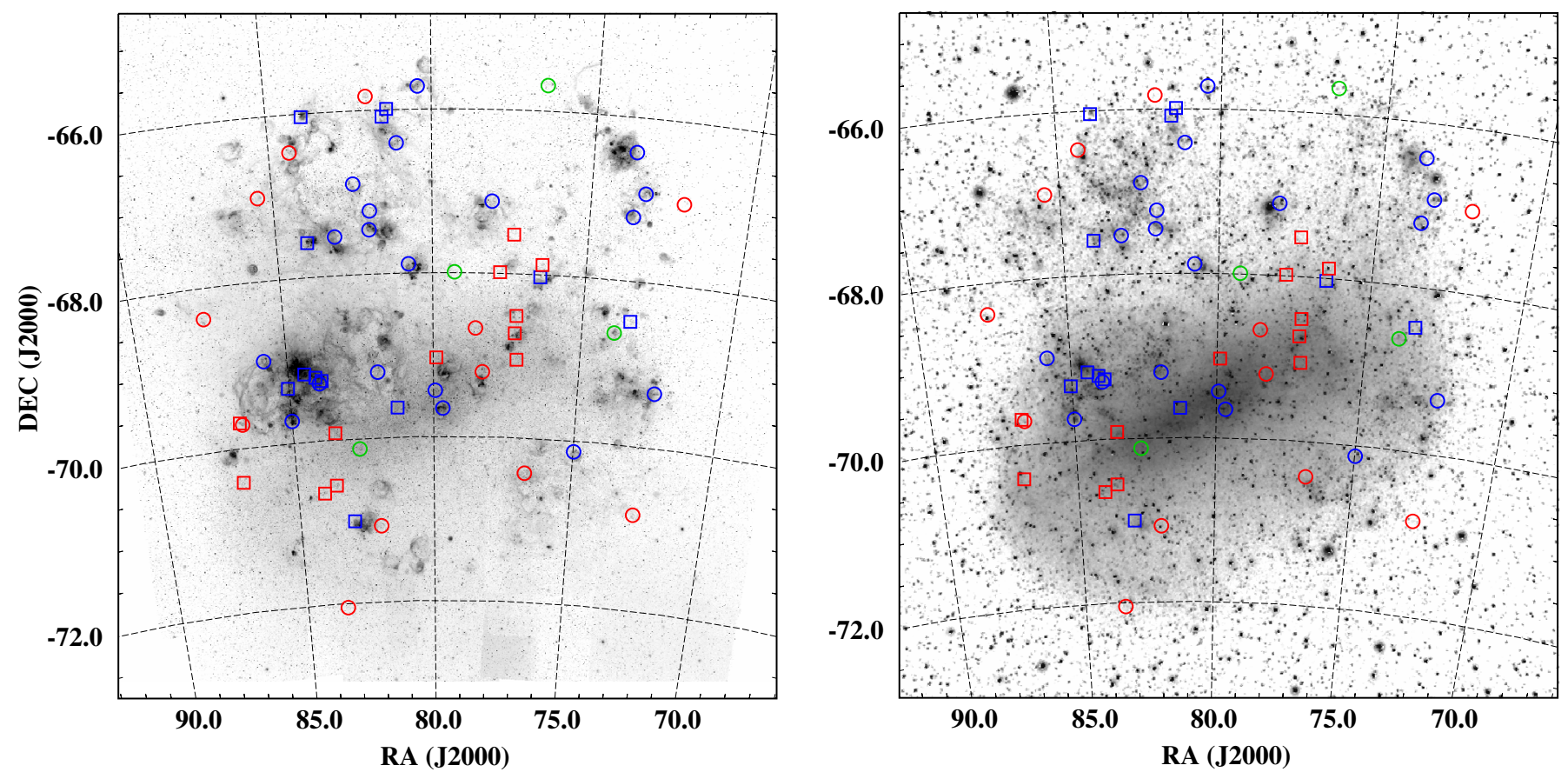

Fig. 13. Left: location of LMC SNRs on the MCELS H $\alpha$ mosaic, displayed logarithmically in greyscale. "Likely-Ia" and "secured-Ia" SNRs are marked by red circles and squares, respectively, while "likely-CC" and "secured-CC" SNRs are shown in blue. Green circles are SNRs with undecided type. Right: same as left on a red continuum image from the SHASSA survey.

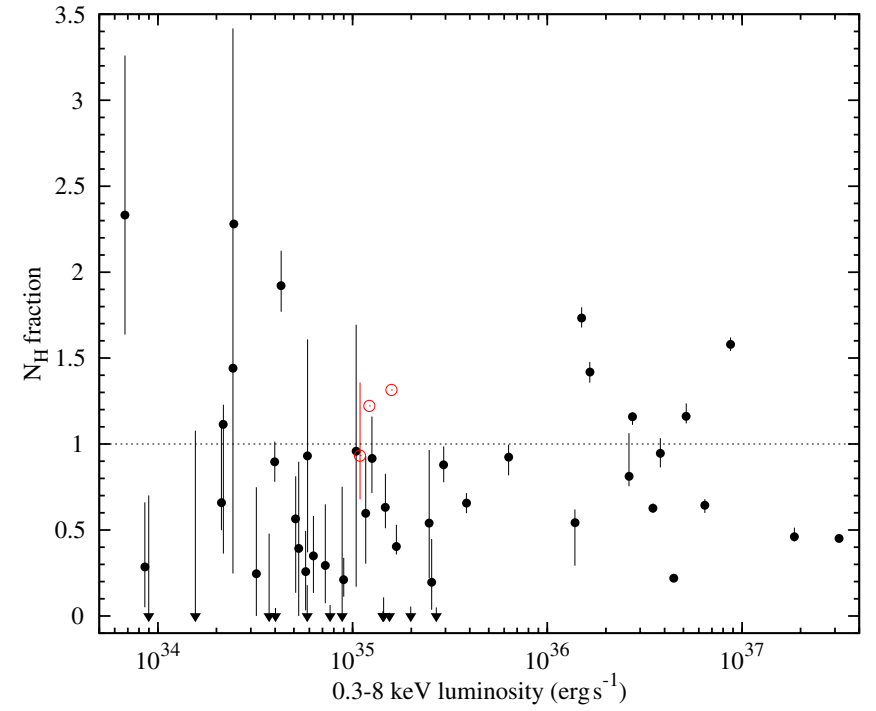

Fig. 14. $N_{\mathrm{H}}$ fraction $=N_{\mathrm{H}}^{\mathrm{X}} / N_{\mathrm{H}}^{21 \mathrm{~cm}}$ as function of broad-band X-ray luminosity (see text for details). Downward pointing arrows indicate upper limits, for objects with $N_{\mathrm{H}}^{\mathrm{X}}$ consistent with 0 . SNRs covered with Chandra are shown in red.

Chandra data only, we obtained $N_{\mathrm{H}}^{\mathrm{X}}$ from the same references as in Sect. 7. Five remaining SNRs have either no or only ROSAT data available, and are not used in this analysis.

$N_{\mathrm{H}}^{21 \mathrm{~cm}}$ is measured from the map of Kim et al. (2003), by averaging the column density around each SNR over a 5' radius (the resolution of the map is about $1^{\prime}$, i.e. $\sim 15 \mathrm{pc}$ ). We checked that using a smaller averaging radius, closer to the typical SNRs size, gave essentially the same results. We then computed the ratio, propagating only the uncertainties on $N_{\mathrm{H}}^{\mathrm{X}}$ since they should dominate the error budget in most cases. $N_{\mathrm{H}}$ fractions are plotted against $L_{\mathrm{X}}$ in Fig. 14. No correlation is evident, as expected: $L_{\mathrm{X}}$ depends mostly on the evolutionary state of the remnant, while the depth within the LMC does not. At lower luminosities, however, there are more remnants with only upper limits on $N_{\mathrm{H}}^{\mathrm{X}}$ (and thus on the $N_{\mathrm{H}}$ fraction). This likely stems from the difficulty of deriving $N_{\mathrm{H}}^{\mathrm{X}}$ from limited X-ray statistics. For the same reason, the error bars are larger in the handful of cases below a few $10^{34} \mathrm{erg} \mathrm{s}^{-1}$, and the sense of depth provided by the $N_{\mathrm{H}}$ fraction becomes blurry.

In Fig. 15, the $N_{\mathrm{H}}$ fraction is projected on the sky, on the same field of view as showed in Figs. 12 and 13. Prominent LMC structures are labelled, including the LMC Bar: the bar is traced by the stars, both in young and intermediate-age populations (de Vaucouleurs \& Freeman 1972; van der Marel 2001, respectively). It was found to be on the near side of the LMC, "floating" $\sim 0.5 \mathrm{kpc}$ to $5 \mathrm{kpc}$ above the plane of the disc, as evidenced from near-infrared star count maps and distances to Cepheids, red clump, and RR Lyrae stars (Zhao \& Evans 2000; Nikolaev et al. 2004; Koerwer 2009; Haschke et al. 2012). This interpretation is challenged by red clump stars distance measurements of Subramaniam \& Subramanian (2009) and Subramanian $\&$ Subramaniam (2013). Zaritsky (2004) proposed an alternative model, where the Bar is a stellar bulge (with a $z$ scale height of $2.5-3 \mathrm{kpc}$ ) whose south-eastern part is obscured by the gas disc. Consequently, the photometric centre is offset (in the plane of the sky), and distance measurements are biased to stars in the near side of the bulge. As can be seen in Fig. 15, SNRs in the Bar region are primarily on the near side (low $N_{\mathrm{H}}$ fraction). Since some of these remnants must originate from the stellar population of the Bar, this lends support to previous findings that the Bar is indeed "floating" in front of the disc. One advantage of our method is that it does not need distance measurements of both disc and Bar objects; it directly gives locations relative to the disc. In the bulge model of Zaritsky (2004), SNRs in the Bar, but behind the 


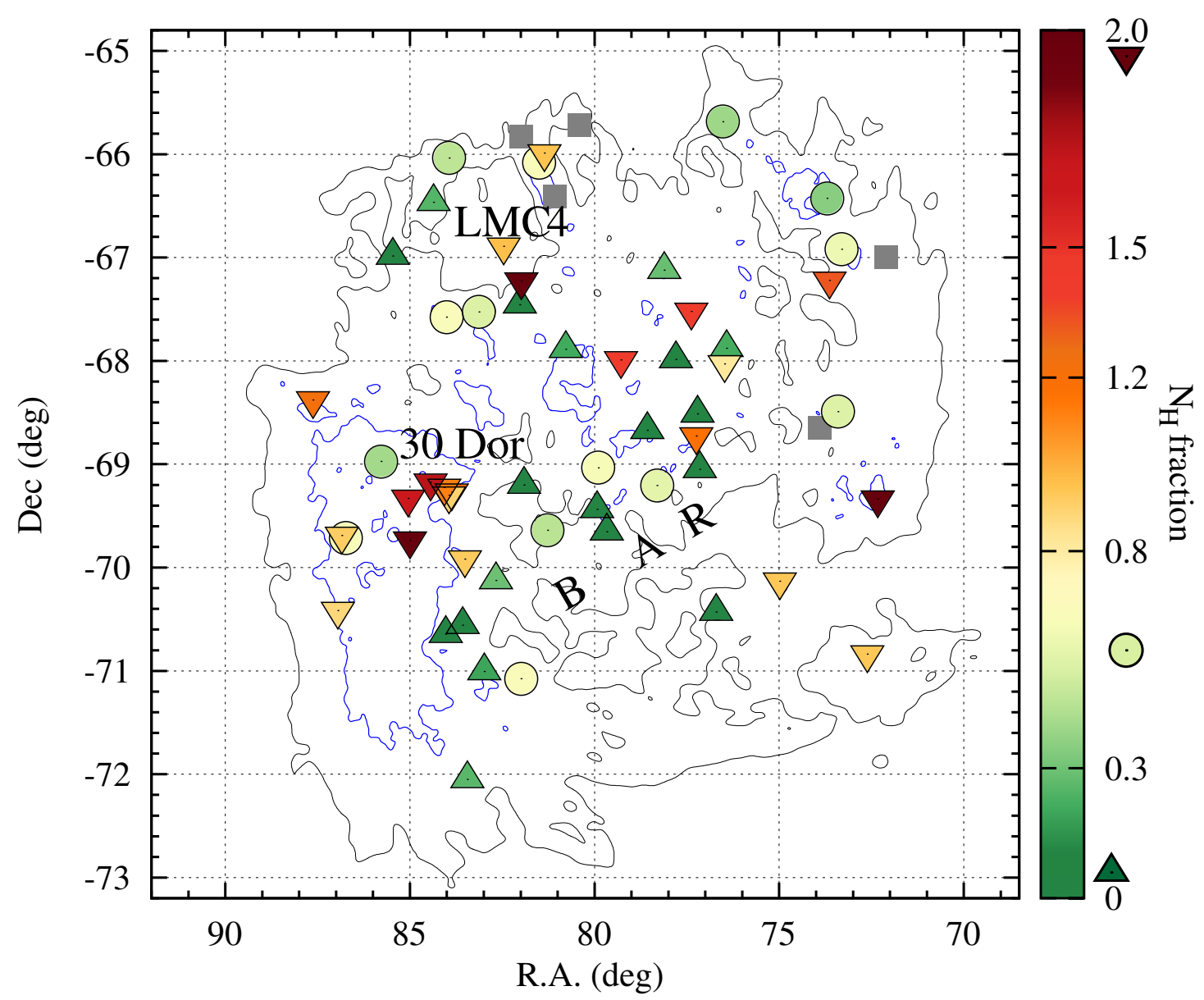

Fig. 15. "Pseudo-3D" distribution of LMC SNRs, using $N_{\mathrm{H}}$ fractions (quantified by the colour bar) as indicators of location along the line of sight. Objects "in front of the disc" $\left(N_{\mathrm{H}}\right.$ fraction $\left.<0.3\right)$ are marked by upward pointing triangles; downward pointing triangles are used for those "behind the disc $\left(N_{\mathrm{H}}\right.$ fraction $\left.>0.8\right)$. Objects within the disc $(0.3$ to 0.8$)$ are marked by dots. The black and blue contours delineate $\mathrm{H}$ I column densities of 1 and $3 \times 10^{21} \mathrm{~cm}^{-2}$, respectively (same as in Fig. 12). Prominent LMC structures are labelled.

disc ${ }^{23}$, should have large $N_{\mathrm{H}}$ fractions, while some scatter should be found along the line of nodes, where the disc and bulge intersect. Unfortunately, there are too few SNRs known in the Bar region to adequately test this alternative model.

The remnants in the 30 Doradus region and directly south of it (MCSNR J0540-6920 and J0540-6944) are the most absorbed, both in absolute and relative terms (largest $N_{\mathrm{H}}^{\mathrm{X}}$ and largest $N_{\mathrm{H}}$ fractions). From distance measurements with red clump stars, Koerwer (2009) found that 30 Dor was further away, although it was noted that this could be an effect of 30 Dor being next to the Bar floating in front of the disc. With our analysis it is confirmed that not only does 30 Dor lie at a larger distance compared to neighbouring features, but is indeed behind the plane of the gas disc.

Finally, it is striking from Figs. 14 and 15 that a few SNRs have an $N_{\mathrm{H}}$ fraction in excess of 1.2 , and up to 2.3. The extra absorption is likely to come from molecular hydrogen in front of the object (Arabadjis \& Bregman 1999). We show in Fig. 12 CO contours from the NANTEN survey (Fukui et al. 2008). CO is used as a tracer of molecular hydrogen. In the east of the LMC there are large regions of molecular gas, following the peak density in $\mathrm{H}$ I. In most cases with large $N_{\mathrm{H}}$ fractions, we find nearby (less than a few arcmin away in projection) $\mathrm{CO}$ clouds, using either the NANTEN catalogue or the higher resolution MAGMA

\footnotetext{
${ }^{23}$ The obscuring effect by the disc on X-rays is moderate, not sufficient to mask SNRs as it does on stars in optical surveys.
}

survey (Fukui et al. 2008; Wong et al. 2011, respectively). We stress that this does not imply that the remnants and the molecular clouds are physically connected, but can be merely a projection effect, with the remnant behind, and not interacting with, the molecular cloud. However, physical interactions can happen, as exemplified by the case of MCSNR J0517-6759, where secondary evidence hints at a physical connection (Maggi et al. 2014).

\section{Summary and outlooks}

We have studied the X-ray emission of the rich population of SNRs in the LMC, using data from the XMM-Newton observatory. We compiled a sample of 59 definite SNRs, cleaned of misclassified objects and doubtful candidates. XMM-Newton data are available for the vast majority (51 SNRs) of the sample, which called for a homogeneous re-analysis of the X-ray spectra of the entire population. This alleviates the inconsistencies in spectral models and analysis methods used, and allows meaningful comparisons of, e.g., temperature, chemical composition, and luminosity of SNRs. The main outcomes of this systematic spectral analysis are the following:

- First, it provides the best census of LMC remnants with an Fe $\mathrm{K}$ line $(\approx 13 \%$ of the sample), which is a powerful tool to retrieve the type of $\mathrm{SN}$ progenitor. 
- Second, it reveals the contribution to the X-ray emission by hot SN ejecta for 23 SNRs ( $\approx 39 \%$ of the sample). Since the abundance ratios measured in the ejecta components reflect the nucleosynthesis yields of either type Ia and CC SNe, this is of great help for the typing of a substantial fraction of the sample.

- And third, it allows us to select 16 SNRs $(\approx 27 \%$ of the sample) where the X-ray emission is dominated by sweptup ISM. In these objects, the fitted abundances provide a measurement of chemical abundances in the gas phase of the LMC ISM. A metallicity of $[\mathrm{Fe} / \mathrm{H}]=-0.46\left({ }_{-0.18}^{+0.13}\right) \mathrm{dex}$ is found based on XMM-Newton SNRs. Light $\alpha$-elements $(\mathrm{O}, \mathrm{Ne}, \mathrm{Mg})$ have lower abundance ratios $[\alpha / \mathrm{Fe}]$ than in the Milky Way. Although this general result was previously known, one can now study abundance ratios within the LMC as function of age. In comparison to old clusters ( $\sim 10 \mathrm{Gyr})$ and red giant stars (1 Gyr and older), the relatively young gas phase ISM ( $\lesssim 100 \mathrm{Myr})$ has a higher metallicity $[\mathrm{Fe} / \mathrm{H}]$ and lower $[\alpha / \mathrm{Fe}]$ (in particular $[\mathrm{O} / \mathrm{Fe}]$ ). This reflects the continued enrichment by type Ia SNe in the last $\sim 1$ Gyr, which injected large amounts of Fe back in the ISM.

We devised a quantitative way to tentatively type all LMC SNRs, based on their local SFHs and stellar environments, combined with spectral information (i.e. detection of SN ejecta, when present). We calibrated this method with SNRs having a wellestablished type based on robust indicators. The resulting ratio of $\mathrm{CC}$ to type Ia SNe that exploded in the LMC over the last few $10^{4}$ yr (i.e. very close to the current ratio of CC/Ia rates) is $N_{\mathrm{CC}} / N_{\text {Ia }}=1.35\left({ }_{-0.24}^{+0.11}\right)$. This is lower than the ratio typically measured in local SNe surveys and in galaxy clusters. After arguing that SNRs of both types might be absent from the sample (i.e. the current sample is not biased towards one type only), we concluded that the low $N_{\mathrm{CC}} / N_{\text {Ia }}$ ratio is a consequence of the specific SFH of the LMC, and particularly the enhanced star formation episodes that occurred $500 \mathrm{Myr}$ and $2 \mathrm{Gyr}$ ago. Because the majority of type Ia SNe explode within 2 Gyr after star-forming episodes, we are coincidentally observing the LMC at a time when the type Ia SN rate is high. Integrated over an SNR lifetime, this results in the relatively low $N_{\mathrm{CC}} / N_{\text {Ia }}$ observed.

We also assessed the spatial distribution of SNRs with respect to cool gas (traced by H I and molecular emission), starforming regions $(\mathrm{H} \alpha)$, and stars (red continuum). A concentration of SNRs around the edge of the SGS LMC 4 exemplifies the role of SGSs in triggering star formation. The column density $N_{\mathrm{H}}^{\mathrm{X}}$ obtained during the X-ray spectral analysis of the whole sample, when compared to the H I column density, provides a measurement of the position of each SNR relative to the H I structure. Since most of the neutral gas lies in a welldefined thin disc seen at a moderate inclination angle, the fraction $N_{\mathrm{H}}^{\mathrm{X}} / N_{\mathrm{H}}^{21 \mathrm{~cm}}$ is a good indicator of the depth along the lineof-sight, revealing the "pseudo-3D" distribution of SNRs in the LMC. Previous studies found that the Bar is "floating" in front of the disc, but this statement was challenged by some authors. Our analysis shows that SNRs in the Bar regions are primarily on the near side (low $N_{\mathrm{H}}$ fraction), lending support to the foreground location of the Bar.

Finally, we compared the populations of SNRs in Local Group galaxies via their X-ray luminosity function. The XLF of SNRs in the SMC, M31, and M33 are relatively homogeneous over all the observed luminosity range, although that of the SMC is flatter. The LMC XLF is remarkable by its prominent bright end. The largest population of SNRs brighter than $L_{\mathrm{X}}>10^{36} \mathrm{erg} \mathrm{s}^{-1}$ is found in the LMC (13 SNRs vs. 8 and
7 in M31 and M33, respectively). This is possibly an effect of the lower metallicity in the LMC: massive stars have smaller mass loss rates (less heavy elements to drive stellar winds) and the interaction of SN ejecta with less massive CSM shells produce brighter remnants. The number of SNRs brighter than $10^{35} \mathrm{erg} \mathrm{s}^{-1}$ in the LMC is comparable to that in M31 and M33, likely owing to its high recent SFR and high current type Ia SN rate. The LMC XLF flattens significantly because of incompleteness: many X-ray-faint SNRs have been missed so far, due to the incomplete coverage of the LMC with sensitive X-ray instruments (i.e. Chandra or XMM-Newton).

This work presents the state of the art on X-ray emission of SNRs in the LMC. However, it is clear that the current sample is incomplete, as evidenced by the flattening of the X-ray luminosity function of LMC SNRs (Sect. 7). In the last 15 years, new SNRs were confirmed or discovered in the LMC at an almost constant rate (one or few per year), principally using X-ray observations. There is no indication that this trend will stop in the near future, so that more observations of the LMC will increase the sample of SNRs.

Nevertheless, the observing time of major observatories is limited and expensive. We conclude this work by offering several strategies to maximise the chance of finding "missing" SNRs:

- As shown in Sect. 8, star formation is intense around the SGS LMC 4, and the edges of the shell abound in SNRs. Many LMC SGS have not been (fully) surveyed by XMM-Newton, for instance (in the notation of Kim et al. 1999) SGS 3 and 6 in the north, SGS 2 and 5 in the west, and SGS 4 in the south. Targeting in particular SGSs associated to star formation (e.g. with H II region along the rims) warrants successful SNR searches.

- The follow-up observations of X-ray-selected candidates (usually ROSAT sources) with XMM-Newton have been extremely successful. Such programmes should be continued until completion of the list of candidates.

- Even the ROSAT (targeted) survey of the LMC was not covering the LMC up to its outskirts. To find SNRs in these regions, the future eROSITA survey (Merloni et al. 2012) will be most useful, covering the full sky in the $0.5-8 \mathrm{keV}$ band. The LMC is located close to the South Ecliptic Pole and will be observed with a deeper exposure than the rest of the sky. Looking for new SNR candidates, especially evolved X-rayonly SNRs, will be of special interest.

Even in existing data, some SNRs might be as yet unrecognised. There is significant diffuse emission from large-scale structures of the hot ISM in the LMC (Sasaki et al. 2002), which is seen in greater spatial and spectral detail by XMM-Newton. By looking for ejecta-enhancement, it might be possible to distinguish old SNRs with low surface brightness hiding in the diffuse emission.

Finding new SNRs is desirable. Individual objects of special interest are often found serendipitously, without prior knowledge of their exciting nature. The evolved type Ia SNRs presented in Maggi et al. (2014) and Bozzetto et al. (2014), are good examples; the discovery of the SNR around the Be/X-ray binary SXP 1062 is another one (Hénault-Brunet et al. 2012; Haberl et al. 2012c). Furthermore, as demonstrated in this work, SNRs are powerful probes of the ISM of their host galaxies. With more SNRs where metallicity can be measured, we will obtain a more accurate knowledge of the chemical composition of the hot ISM or better assess its homogeneity.

Acknowledgements. We thank the anonymous referee for carefully reading this rather long manuscript and providing us with comments and suggestions to 
improve it. The XMM-Newton project is supported by the Bundesministerium für Wirtschaft und Technologie/Deutsches Zentrum für Luft- und Raumfahrt (BMWi/DLR, FKZ 50 OX 0001) and the Max-Planck Society. P.M. acknowledges support by the Centre National d'Études Spatiales (CNES) and from the BMWi/DLR grant FKZ 50 OR 1201. M.S. acknowledges support by the Deutsche Forschungsgemeinschaft through the Emmy Noether Research Grant SA 2131/1-1. P.K. acknowledges support from the BMWi/DLR grant FKZ 50 OR 1309. This research has made use of Aladin, SIMBAD and VizieR, operated at the CDS, Strasbourg, France.

\section{References}

Abbey, T., Carpenter, J., Read, A., et al. 2006, in The X-ray Universe 2005, ed. A. Wilson, ESA Spec. Publ., 604, 943

Arabadjis, J. S., \& Bregman, J. N. 1999, ApJ, 510, 806

Arnaud, K. A. 1996, in Astronomical Data Analysis Software and Systems V, eds. G. H. Jacoby \& J. Barnes, ASP Conf. Ser., 101, 17

Badenes, C., Bravo, E., Borkowski, K. J., \& Domínguez, I. 2003, ApJ, 593, 358

Badenes, C., Borkowski, K. J., \& Bravo, E. 2005, ApJ, 624, 198

Badenes, C., Hughes, J. P., Cassam-Chenaï, G., \& Bravo, E. 2008, ApJ, 680, 1149

Badenes, C., Harris, J., Zaritsky, D., \& Prieto, J. L. 2009, ApJ, 700, 727

Badenes, C., Maoz, D., \& Draine, B. T. 2010, MNRAS, 407, 1301

Balucinska-Church, M., \& McCammon, D. 1992, ApJ, 400, 699

Bamba, A., Ueno, M., Nakajima, H., \& Koyama, K. 2004, ApJ, 602, 257

Bamba, A., Yamazaki, R., Yoshida, T., Terasawa, T., \& Koyama, K. 2005, ApJ, 621,793

Bamba, A., Ueno, M., Nakajima, H., Mori, K., \& Koyama, K. 2006, A\&A, 450, 585

Barret, D., den Herder, J. W., Piro, L., et al. 2013, ArXiv e-prints [arXiv: 1308.6784]

Behar, E., Rasmussen, A. P., Griffiths, R. G., et al. 2001, A\&A, 365, L242

Blackburn, J. K. 1995, in Astronomical Data Analysis Software and Systems IV, eds. R. A. Shaw, H. E. Payne, \& J. J. E. Hayes, ASP Conf. Ser., 77, 367

Blair, W. P., Ghavamian, P., Sankrit, R., \& Danforth, C. W. 2006, ApJS, 165, 480

Bojičić, I. S., Filipović, M. D., Parker, Q. A., et al. 2007, MNRAS, 378, 1237

Book, L. G., Chu, Y.-H., Gruendl, R. A., \& Fukui, Y. 2009, AJ, 137, 3599

Borkowski, K. J., Hendrick, S. P., \& Reynolds, S. P. 2006, ApJ, 652, 1259

Borkowski, K. J., Hendrick, S. P., \& Reynolds, S. P. 2007, ApJ, 671, L45

Bozzetto, L. M., Filipović, M. D., Crawford, E. J., et al. 2012a, MNRAS, 420, 2588

Bozzetto, L. M., Filipovic, M. D., Crawford, E. J., et al. 2012b, Rev. Mex. Astron. Astrofis., 48, 41

Bozzetto, L. M., Filipović, M. D., Crawford, E. J., et al. 2013, MNRAS, 432, 2177

Bozzetto, L. M., Kavanagh, P. J., Maggi, P., et al. 2014, MNRAS, 439, 1110

Cajko, K. O., Crawford, E. J., \& Filipovic, M. D. 2009, Serbian Astronomical J., 179,55

Cash, W. 1979, ApJ, 228, 939

Chen, L.-W., Fabian, A. C., \& Gendreau, K. C. 1997, MNRAS, 285, 449

Chen, Y., Wang, Q. D., Gotthelf, E. V., et al. 2006, ApJ, 651, 237

Chevalier, R. A., \& Kirshner, R. P. 1979, ApJ, 233, 154

Chevalier, R. A., \& Raymond, J. C. 1978, ApJ, 225, L27

Chevalier, R. A., Kirshner, R. P., \& Raymond, J. C. 1980, ApJ, 235, 186

Chu, Y.-H. 2008, in IAU Symp. 250, eds. F. Bresolin, P. A. Crowther, \& J. Puls, 341

Chu, Y.-H., \& Kennicutt, Jr., R. C. 1988, AJ, 96, 1874

Chu, Y.-H., Mac Low, M.-M., Garcia-Segura, G., Wakker, B., \& Kennicutt, Jr., R. C. 1993, ApJ, 414, 213

Chu, Y.-H., Dickel, J. R., Staveley-Smith, L., Osterberg, J., \& Smith, R. C. 1995, AJ, 109, 1729

Chu, Y.-H., Kennicutt, R. C., Snowden, S. L., et al. 1997, PASP, 109, 554

Chu, Y.-H., Kim, S., Points, S. D., Petre, R., \& Snowden, S. L. 2000, AJ, 119, 2242

Cole, A. A., Tolstoy, E., Gallagher, III, J. S., \& Smecker-Hane, T. A. 2005, AJ, 129,1465

Corbelli, E. 2003, MNRAS, 342, 199

Crawford, E. J., Filipović, M. D., McEntaffer, R. L., et al. 2014, AJ, 148, 99

Da Costa, G. S. 1991, in The Magellanic Clouds, eds. R. Haynes, \& D. Milne, IAU Symp., 148, 183

de Grijs, R., Wicker, J. E., \& Bono, G. 2014, AJ, 147, 122

de Horta, A. Y., Filipović, M. D., Bozzetto, L. M., et al. 2012, A\&A, 540, A25

de Plaa, J., Werner, N., Bleeker, J. A. M., et al. 2007, A\&A, 465, 345

de Vaucouleurs, G., \& Freeman, K. C. 1972, Vistas Astron., 14, 163

den Herder, J. W., Brinkman, A. C., Kahn, S. M., et al. 2001, A\&A, 365, L7

Dennerl, K., Haberl, F., Aschenbach, B., et al. 2001, A\&A, 365, L202

Desai, K. M., Chu, Y.-H., Gruendl, R. A., et al. 2010, AJ, 140, 584
Dickey, J. M., \& Lockman, F. J. 1990, ARA\&A, 28, 215

Dopita, M. A., Blair, W. P., Long, K. S., et al. 2010, ApJ, 710, 964

Dunne, B. C., Points, S. D., \& Chu, Y.-H. 2001, ApJS, 136, 119

Dwarkadas, V. V. 2005, ApJ, 630, 892

Dwarkadas, V. V. 2007, ApJ, 667, 226

Eisenhardt, P. R. M., Brodwin, M., Gonzalez, A. H., et al. 2008, ApJ, 684, 905

Elmegreen, B. G. 1998, in Origins, ASP Conf. Ser. 148, 150, eds. C. E.

Woodward, J. M. Shull, \& H. A. Thronson, Jr.

Ferrand, G., \& Safi-Harb, S. 2012, Adva. Space Res., 49, 1313

Filipovic, M. D., Haynes, R. F., White, G. L., \& Jones, P. A. 1998, A\&AS, 130, 421

Filipović, M. D., Haberl, F., Winkler, P. F., et al. 2008, A\&A, 485, 63

Flanagan, K. A., Canizares, C. R., Dewey, D., et al. 2004, ApJ, 605, 230

Freyberg, M. J., Briel, U. G., Dennerl, K., et al. 2004, in SPIE Conf. Ser. 5165, eds. K. A. Flanagan, \& O. H. W. Siegmund, 112

Fukui, Y., Kawamura, A., Minamidani, T., et al. 2008, ApJS, 178, 56

Gaensler, B. M., Slane, P. O., Gotthelf, E. V., \& Vasisht, G. 2001, ApJ, 559, 963

Gaensler, B. M., Hendrick, S. P., Reynolds, S. P., \& Borkowski, K. J. 2003, ApJ, 594, L111

Gaustad, J. E., McCullough, P. R., Rosing, W., \& Van Buren, D. 2001, PASP, 113,1326

Ghavamian, P., Rakowski, C. E., Hughes, J. P., \& Williams, T. B. 2003, ApJ, 590, 833

Green, D. A. 2014, Bull. Astron. Soc. India, 42, 47

Grondin, M.-H., Sasaki, M., Haberl, F., et al. 2012, A\&A, 539, A15

Haberl, F., \& Pietsch, W. 1999, A\&AS, 139, 277

Haberl, F., Geppert, U., Aschenbach, B., \& Hasinger, G. 2006, A\&A, 460, 811

Haberl, F., Filipović, M. D., Bozzetto, L. M., et al. 2012a, A\&A, 543, A154

Haberl, F., Sturm, R., Ballet, J., et al. 2012b, A\&A, 545, A128

Haberl, F., Sturm, R., Filipović, M. D., Pietsch, W., \& Crawford, E. J. 2012c, A\&A, 537, L1

Harris, J., \& Zaritsky, D. 2004, AJ, 127, 1531

Harris, J., \& Zaritsky, D. 2006, AJ, 131, 2514

Harris, J., \& Zaritsky, D. 2008, PASA, 25, 116

Harris, J., \& Zaritsky, D. 2009, AJ, 138, 1243

Haschke, R., Grebel, E. K., \& Duffau, S. 2012, AJ, 144, 106

Helder, E. A., Broos, P. S., Dewey, D., et al. 2013, ApJ, 764, 11

Hénault-Brunet, V., Oskinova, L. M., Guerrero, M. A., et al. 2012, MNRAS, 420 , L13

Hendrick, S. P., Borkowski, K. J., \& Reynolds, S. P. 2003, ApJ, 593, 370

Heng, K., Haberl, F., Aschenbach, B., \& Hasinger, G. 2008, ApJ, 676, 361

Henley, D. B., \& Shelton, R. L. 2008, ApJ, 676, 335

Hodge, P. W. 1960, ApJ, 131, 351

Hodge, P. W. 1961, ApJ, 133, 413

Holtzman, J. A., Gallagher, III, J. S., Cole, A. A., et al. 1999, AJ, 118, 2262

Hughes, J. P., Hayashi, I., Helfand, D., et al. 1995, ApJ, 444, L81

Hughes, J. P., Hayashi, I., \& Koyama, K. 1998, ApJ, 505, 732

Hughes, J. P., Ghavamian, P., Rakowski, C. E., \& Slane, P. O. 2003, ApJ, 582, L95

Hughes, J. P., Rafelski, M., Warren, J. S., et al. 2006, ApJ, 645, L117

Jansen, F., Lumb, D., Altieri, B., et al. 2001, A\&A, 365, L1

Jaskot, A. E., Strickland, D. K., Oey, M. S., Chu, Y.-H., \& García-Segura, G. 2011, ApJ, 729, 28

Jennings, Z. G., Williams, B. F., Murphy, J. W., et al. 2012, ApJ, 761, 26 Jennings, Z. G., Williams, B. F., Murphy, J. W., et al. 2014, ApJ, 795, 170

Johnson, J. A., Ivans, I. I., \& Stetson, P. B. 2006, ApJ, 640, 801

Kaaret, P., Marshall, H. L., Aldcroft, T. L., et al. 2001, ApJ, 546, 1159

Kaplan, D. L., Kulkarni, S. R., van Kerkwijk, M. H., et al. 2001, ApJ, 556, 399

Kavanagh, P. J., Sasaki, M., Points, S. D., et al. 2013, A\&A, 549, A99

Kavanagh, P. J., Sasaki, M., Bozzetto, L. M., et al. 2015a, A\&A, 573, A73

Kavanagh, P. J., Sasaki, M., Whelan, E. T., et al. 2015b, A\&A, 583, A121

Kavanagh, P. J., Sasaki, M., Bozzetto, L. M., et al. 2015c, A\&A, 579, A63

Kim, S., Dopita, M. A., Staveley-Smith, L., \& Bessell, M. S. 1999, AJ, 118, 2797

Kim, S., Staveley-Smith, L., Dopita, M. A., et al. 2003, ApJS, 148, 473

Klimek, M. D., Points, S. D., Smith, R. C., Shelton, R. L., \& Williams, R. 2010, ApJ, 725, 2281

Koerwer, J. F. 2009, AJ, 138,

Kosenko, D., Vink, J., Blinnikov, S., \& Rasmussen, A. 2008, A\&A, 490, 223

Kosenko, D., Helder, E. A., \& Vink, J. 2010, A\&A, 519, A11

Koyama, K., Petre, R., Gotthelf, E. V., et al. 1995, Nature, 378, 255

Kozłowski, S., Kochanek, C. S., Jacyszyn, A. M., et al. 2012, ApJ, 746, 27

Kudritzki, R.-P., \& Puls, J. 2000, ARA\&A, 38, 613

Kuntz, K. D., \& Snowden, S. L. 2008, A\&A, 478, 575

Kuntz, K. D., \& Snowden, S. L. 2010, ApJS, 188, 46

Lapenna, E., Mucciarelli, A., Origlia, L., \& Ferraro, F. R. 2012, ApJ, 761, 33

Lasker, B. M. 1978, ApJ, 223, 109

Lejeune, T., \& Schaerer, D. 2001, A\&A, 366, 538

Lewis, K. T., Burrows, D. N., Hughes, J. P., et al. 2003, ApJ, 582, 770 
Li, W., Leaman, J., Chornock, R, et al. 2011, MNRAS, 412, 1441 Long, K. S., Helfand, D. J., \& Grabelsky, D. A. 1981, ApJ, 248, 925 Long, K. S., Blair, W. P., Winkler, P. F., et al. 2010, ApJS, 187, 495 Lopez, L. A., Ramirez-Ruiz, E., Badenes, C., et al. 2009, ApJ, 706, L106 Lopez, L. A., Ramirez-Ruiz, E., Huppenkothen, D., Badenes, C., \& Pooley, D. A. 2011, ApJ, 732, 114

Lovisari, L., Schindler, S., \& Kapferer, W. 2011, A\&A, 528, A60 Lowry, J. D., Chu, Y.-H., Guerrero, M. A., et al. 2004, AJ, 127, 125 Lumb, D. H., Warwick, R. S., Page, M., \& De Luca, A. 2002, A\&A, 389, 93 Lumb, D. H., Schartel, N., \& Jansen, F. A. 2012, Opt. Eng., 51, 011009 Mac Low, M.-M., \& Klessen, R. S. 2004, Reviews of Modern Physics, 76, 125 Maggi, P., Haberl, F., Bozzetto, L. M., et al. 2012a, A\&A, 546, A109 Maggi, P., Haberl, F., Sturm, R., \& Dewey, D. 2012b, A\&A, 548, L3 Maggi, P., Haberl, F., Kavanagh, P. J., et al. 2014, A\&A, 561, A76 Maoz, D. 2008, MNRAS, 384, 267

Maoz, D., \& Badenes, C. 2010, MNRAS, 407, 1314

Maoz, D., \& Mannucci, F. 2012, PASA, 29, 447

Mason, K. O., Breeveld, A., Much, R., et al. 2001, A\&A, 365, L36 Mathewson, D. S., \& Clarke, J. N. 1973, ApJ, 180, 725

Mathewson, D. S., Healey, J. R., \& Westerlund, B. E. 1963, Nature, 199, 681 Mathewson, D. S., Ford, V. L., Dopita, M. A., et al. 1983, ApJS, 51, 345 Mathewson, D. S., Ford, V. L., Dopita, M. A., et al. 1984, ApJS, 55, 189

Mathewson, D. S., Ford, V. L., Tuohy, I. R., et al. 1985, ApJS, 58, 197

Meaburn, J. 1980, MNRAS, 192, 365

Merloni, A., Predehl, P., Becker, W., et al. 2012, ArXiv e-prints [arXiv: 1209.3114]

Mills, B. Y., Turtle, A. J., Little, A. G., \& Durdin, J. M. 1984, Austr. J. Phys., 37, 321

Misanovic, Z., Pietsch, W., Haberl, F., et al. 2006, A\&A, 448, 1247

Mokiem, M. R., de Koter, A., Vink, J. S., et al. 2007, A\&A, 473, 603

Morse, J. A., Winkler, P. F., \& Kirshner, R. P. 1995, AJ, 109, 2104

Nikolaev, S., Drake, A. J., Keller, S. C., et al. 2004, ApJ, 601, 260

Nishiuchi, M., Yokogawa, J., Koyama, K., \& Hughes, J. P. 2001, PASJ, 53,99

Owen, R. A., Filipović, M. D., Ballet, J., et al. 2011, A\&A, 530, A132

Park, S., Burrows, D. N., Garmire, G. P., et al. 2003a, ApJ, 586, 210

Park, S., Hughes, J. P., Slane, P. O., et al. 2003b, apj1, 592, L41

Park, S., Hughes, J. P., Slane, P. O., et al. 2012, ApJ, 748, 117

Payne, J. L., White, G. L., \& Filipović, M. D. 2008, MNRAS, 383, 1175

Peñarrubia, J., Ma, Y.-Z., Walker, M. G., \& McConnachie, A. 2014, MNRAS, 443, 2204

Perlmutter, S., Aldering, G., Goldhaber, G., et al. 1999, ApJ, 517, 565

Peters, C. L., Lopez, L. A., Ramirez-Ruiz, E., Stassun, K. G., \& FigueroaFeliciano, E. 2013, ApJ, 771, L38

Pietrzyński, G., Graczyk, D., Gieren, W., et al. 2013, Nature, 495, 76

Pietsch, W., Misanovic, Z., Haberl, F., et al. 2004, A\&A, 426, 11

Pietsch, W., Freyberg, M., \& Haberl, F. 2005, A\&A, 434, 483

Plucinsky, P. P., Williams, B., Long, K. S., et al. 2008, ApJS, 174, 366

Pompéia, L., Hill, V., Spite, M., et al. 2008, A\&A, 480, 379

Rest, A., Suntzeff, N. B., Olsen, K., et al. 2005, Nature, 438, 1132

Rest, A., Matheson, T., Blondin, S., et al. 2008, ApJ, 680, 1137

Reyes-Iturbide, J., Rosado, M., \& Velázquez, P. F. 2008, AJ, 136, 2011

Reyes-Iturbide, J., Rosado, M., Rodríguez-González, A., et al. 2014, AJ, 148, 102

Rho, J., Dyer, K. K., Borkowski, K. J., \& Reynolds, S. P. 2002, ApJ, 581, 1116 Riess, A. G., Filippenko, A. V., Challis, P., et al. 1998, AJ, 116, 1009

Russell, S. C., \& Dopita, M. A. 1992, ApJ, 384, 508

Sasaki, M., Haberl, F., \& Pietsch, W. 2002, A\&A, 392, 103

Sasaki, M., Pietsch, W., Haberl, F., et al. 2012, A\&A, 544, A144

Sato, K., Tokoi, K., Matsushita, K., et al. 2007, ApJ, 667, L41

Seok, J. Y., Koo, B.-C., Onaka, T., et al. 2008, PASJ, 60, 453

Seok, J. Y., Koo, B.-C., \& Onaka, T. 2013, ApJ, 779, 134

Seward, F. D., Williams, R. M., Chu, Y.-H., et al. 2006, ApJ, 640, 327

Seward, F. D., Charles, P. A., Foster, D. L., et al. 2012, ApJ, 759, 123

Sim, S. A., Röpke, F. K., Hillebrandt, W., et al. 2010, ApJ, 714, L52

Slane, P. 2014, in IAU Symp. 296, eds. A. Ray, \& R. A. McCray, 226

Smecker-Hane, T. A., Cole, A. A., Gallagher, III, J. S., \& Stetson, P. B. 2002, ApJ, 566, 239
Smith, R. C., Chu, Y.-H., Mac Low, M.-M., Oey, M. S., \& Klein, U. 1994, AJ, 108, 1266

Smith, C., Leiton, R., \& Pizarro, S. 2000, in Stars, Gas and Dust in Galaxies: Exploring the Links, eds. D. Alloin, K. Olsen, \& G. Galaz, ASP Conf. Ser., 221,83

Snowden, S. L., Mushotzky, R. F., Kuntz, K. D., \& Davis, D. S. 2008, A\&A, 478,615

Stanimirović, S., Staveley-Smith, L., \& Jones, P. A. 2004, ApJ, 604, 176

Staveley-Smith, L., Kim, S., Calabretta, M. R., Haynes, R. F., \& Kesteven, M. J. 2003, MNRAS, 339, 87

Stiele, H., Pietsch, W., Haberl, F., et al. 2011, A\&A, 534, A55

Strüder, L., Briel, U., Dennerl, K., et al. 2001, A\&A, 365, L18

Sturm, R. K. N. 2012, PhD thesis, Fakultät für Physik, Technische Universität München, Germany

Sturm, R., Haberl, F., Aschenbach, B., \& Hasinger, G. 2010, A\&A, 515, A5

Sturm, R., Haberl, F., Pietsch, W., et al. 2013, A\&A, 558, A3

Subramaniam, A., \& Subramanian, S. 2009, ApJ, 703, L37

Subramanian, S., \& Subramaniam, A. 2013, A\&A, 552, A144

Tabatabaei, F. S., \& Berkhuijsen, E. M. 2010, A\&A, 517, A77

Takahashi, T., Mitsuda, K., Kelley, R., et al. 2012, in SPIE Conf. Ser., 8443

Tsujimoto, T., Nomoto, K., Yoshii, Y., et al. 1995, MNRAS, 277, 945

Tüllmann, R., Gaetz, T. J., Plucinsky, P. P., et al. 2011, ApJS, 193, 31

Tuohy, I. R., Dopita, M. A., Mathewson, D. S., Long, K. S., \& Helfand, D. J. 1982, ApJ, 261, 473

Turner, M. J. L., Abbey, A., Arnaud, M., et al. 2001, A\&A, 365, L27

Turtle, A. J., \& Mills, B. Y. 1984, PASA, 5, 537

van der Heyden, K. J., Behar, E., Vink, J., et al. 2002, A\&A, 392, 955

van der Heyden, K. J., Bleeker, J. A. M., Kaastra, J. S., \& Vink, J. 2003, A\&A, 406,141

van der Heyden, K. J., Bleeker, J. A. M., \& Kaastra, J. S. 2004, A\&A, 421, 1031 van der Marel, R. P. 2001, AJ, 122, 1827

van der Marel, R. P., \& Kallivayalil, N. 2014, ApJ, 781, 121

van der Marel, R. P., Alves, D. R., Hardy, E., \& Suntzeff, N. B. 2002, AJ, 124, 2639

Van der Swaelmen, M., Hill, V., Primas, F., \& Cole, A. A. 2013, A\&A, 560, A44

van Kerkwijk, M. H., Chang, P., \& Justham, S. 2010, ApJ, 722, L157

Verley, S., Corbelli, E., Giovanardi, C., \& Hunt, L. K. 2009, A\&A, 493, 453

Vink, J. 2012, A\&ARv, 20, 49

Vink, J. S., de Koter, A., \& Lamers, H. J. G. L. M. 2001, A\&A, 369, 574

Vogt, F. P. A., Besel, M.-A., Krause, O., \& Dullemond, C. P. 2012, ApJ, 750, 155

Wang, Q., \& Helfand, D. J. 1991, ApJ, 373, 497

Warren, J. S., \& Hughes, J. P. 2004, ApJ, 608, 261

Warren, J. S., Hughes, J. P., \& Slane, P. O. 2003, ApJ, 583, 260

Warth, G., Sasaki, M., Kavanagh, P. J., et al. 2014, A\&A, 567, A136

Watson, M. G., Schröder, A. C., Fyfe, D., et al. 2009, A\&A, 493, 339

Westerlund, B. E. 1997, The Magellanic Clouds (Cambridge University Press)

Westerlund, B. E., \& Mathewson, D. S. 1966, MNRAS, 131, 371

Williams, R. M., \& Chu, Y.-H. 2005, ApJ, 635, 1077

Williams, R. M., Chu, Y.-H., Dickel, J. R., et al. 1999a, ApJS, 123, 467

Williams, R. M., Chu, Y.-H., Dickel, J. R., et al. 1999b, ApJ, 514, 798

Williams, R. M., Chu, Y.-H., Dickel, J. R., et al. 2005, ApJ, 628, 704

Williams, B. F., Wold, B., Haberl, F., et al. 2015, ApJS, 218, 9

Wilms, J., Allen, A., \& McCray, R. 2000, ApJ, 542, 914

Wong, T., Hughes, A., Ott, J., et al. 2011, ApJS, 197, 16

Woosley, S. E., \& Kasen, D. 2011, ApJ, 734, 38

Yamaguchi, R., Mizuno, N., Onishi, T., Mizuno, A., \& Fukui, Y. 2001a, ApJ, 553, L185

Yamaguchi, R., Mizuno, N., Onishi, T., Mizuno, A., \& Fukui, Y. 2001b, PASJ, 53, 959

Yamaguchi, H., Badenes, C., Petre, R., et al. 2014, ApJ, 785, L27

Zaritsky, D. 2004, ApJ, 614, L37

Zaritsky, D., Harris, J., Thompson, I. B., \& Grebel, E. K. 2004, AJ, 128, 1606

Zhang, N.-X., Chu, Y.-H., Williams, R. M., et al. 2014, ApJ, 792, 58

Zhao, H., \& Evans, N. W. 2000, ApJ, 545, L35 


\section{Appendix A: The EPIC background}

The signal recorded in an XMM-Newton observation comprises many components, which can be separated into three main groups: the X-ray emission of the target of the observation, an astrophysical X-ray background (hereafter AXB, i.e. X-rays from any source located in projection near the target), and an instrumental background. In this Appendix we describe the last two components.

The instrumental background of EPIC: it consists of three components. The first is an electronic noise, in the form of hot pixels/columns or read-out noise. In the case of EPIC$\mathrm{pn}$, the read-out noise increases dramatically below energies of $\$ 300 \mathrm{eV}$, especially if double-pixel events are used.

The second component is the particle-induced background, the spectrum of which includes both a continuum and many lines. The continuum part is due to the quiescent particle background (QPB). Cosmic rays deposit a large amount of energy $(\gg 10 \mathrm{keV})$ in many adjacent pixels and are easy to distinguish from valid X-ray events. However, the unrejected fraction of direct and Compton-scattered cosmic rays produces a remaining continuum with a rather flat spectrum and a rate of $0.021 \pm 0.0022$ events $\mathrm{cm}^{-2} \mathrm{~s}^{-1}$ for the MOS cameras, and about twice as much for pn (Lumb et al. 2002). The continuum is both chip-, position-, and time-dependent (at least for MOS where it has been extensively studied, see Kuntz \& Snowden 2008). These variations have to be taken into account in the spectral analysis. The line part of the particle background is composed of many X-ray fluorescence lines produced by the interaction of high-energy particles with the material surrounding the detectors $(\mathrm{Al}, \mathrm{Ni}, \mathrm{Cu}$, etc.). Due to this origin the fluorescence line component varies with time. This component is highly positiondependent, mirroring the distribution of the camera material around the detectors (Lumb et al. 2002; Freyberg et al. 2004; Kuntz \& Snowden 2008).

The third component is the so-called soft proton contamination (SPC). Low-energy protons, accelerated in the Earth magnetosphere and focused by the X-ray telescopes onto the detectors, produce events that cannot be distinguished from genuine X-ray events. The soft proton flux has a highly time-variable, "flaring" nature. At times of the strongest flares, most of the data are unusable anyway (except in the case of a very bright target). But soft proton flares can occur on longer time scales, at lower amplitudes. These time intervals are typically used for science, though they include a small but potentially important contamination by soft protons (hence the term "SPC"). The flaring spectrum was found by Kuntz \& Snowden (2008) to be rather flat, with a small roll-off at high energy. The same authors showed that the stronger the flare, the flatter (i.e. harder) the SPC spectrum.

The contribution of the instrumental background will be relatively higher in spectra of sources with low surface brightness and must be taken into account, i.e. modelled. To do so, we use the FWC data (Sect. 2.2). Several hundred kiloseconds worth of data are now available, providing a good knowledge of the spectrum of the instrumental background. As part of his $\mathrm{PhD}$ thesis, Richard Sturm (2012) developed an empirical model of the EPIC-pn FWC data. This includes an exponential decay (modified by a spline function), a power law, and a combination of Gaussian lines to account for the electronic noise, QPB, and instrumental lines, respectively. In addition, two smeared absorption edges to the continuum are included.

We extended his work and developed a similar model for the EPIC-MOS FWC spectra. This allows joint analyses of the pn and MOS spectra of LMC SNRs and take advantage of the better spectral resolution of MOS. There is no low-energy noise as for EPIC-pn, so no exponential decay function is needed. Satisfactory results were obtained with a broken power law for the continuum, leaving the slope of the two segments as well as the energy of the break free. A smeared absorption edge around $E \approx 0.53 \mathrm{keV}$ (K edge of oxygen) improved the fit and was included. A set of Gaussian are used to model the fluorescence lines. The materials of the MOS and pn cameras are different, and so is the observed fluorescence line pattern. Both have a strong $\mathrm{Al} \mathrm{K}$ line at $1.49 \mathrm{keV}$. MOS background also features a strong $\mathrm{Si} \mathrm{K}$ line at $1.74 \mathrm{keV}$, as opposed to pn, where the strongest line is $\mathrm{Cu} \mathrm{K}$ at $8.05 \mathrm{keV}$. Other lines from $\mathrm{Au}, \mathrm{Cr}$, $\mathrm{Mn}, \mathrm{Zn}, \mathrm{Cu}, \mathrm{Fe}$, and $\mathrm{Ni}$ are detected (Lumb et al. 2002; Kuntz $\&$ Snowden 2008) and included in the MOS detector background model.

Note that the same screening and filtering criteria used for the science data are applied to the FWC data, including the vignetting correction with evigweight (Sect. 4.2.2). Formally, the instrumental background is not subjected to vignetting, which is an effect of the telescope on photons. However, by applying a vignetting correction to the science data, one assigns weights to genuine X-ray events as well as to particle background events, since one cannot a priori distinguish the two type of events. Therefore, one needs to vignetting-correct the FWC data to make sure that the FWC spectra, used for the modelling of the instrumental background contribution to the science data, have been processed in the same way as the latter. At a given position on the detector, evigweight will assign heavier weights to photons with higher energies, an effect that can be easily accounted for in the background model. To do so, we added a spline function to the pn and MOS instrumental background models. This reproduces the effect of the vignetting correction, which "overweights" events above $5 \mathrm{keV}$, if they have been recorded at significant off-axis angles.

The astrophysical X-ray background: the AXB can usually be modelled with four or less components (Snowden et al. 2008; Kuntz \& Snowden 2010). The soft part of the AXB $(E \lesssim 2 \mathrm{keV})$ has mostly a thermal emission spectrum and originates from various regions/hot plasmas. The Local Hot Bubble (LHB) is a region in the solar neighbourhood filled with million-degree plasma $(k T \approx 85 \mathrm{eV}$, Henley \& Shelton 2008). This component was modelled with an unabsorbed APEC model. Emission from the Galactic halo comprises a cool $(k T \sim 0.1 \mathrm{keV})$ and warm $(k T \sim 0.25 \mathrm{keV})$ thermal component. Since the cool component is mostly absorbed by the foreground Galactic absorbing column, we did not include it in the AXB model to keep it as simple as possible. For the warm component we used an absorbed APEC model.

Above $2 \mathrm{keV}$, the background is mostly from the cosmic $\mathrm{X}$-ray background (CXB), a superposition of unresolved distant objects, in other words AGN. This component has an absorbed power-law spectrum, with a photon index fixed at $\Gamma=1.46$ (Chen et al. 1997). To account for the non-uniformity of this component, the normalisation is a free parameter in our background model.

Note: Cases with a problematic background Several situations can occur where the instrumental and X-ray backgrounds cannot be properly accounted for at first, hindering the analysis of the SNR emission. 


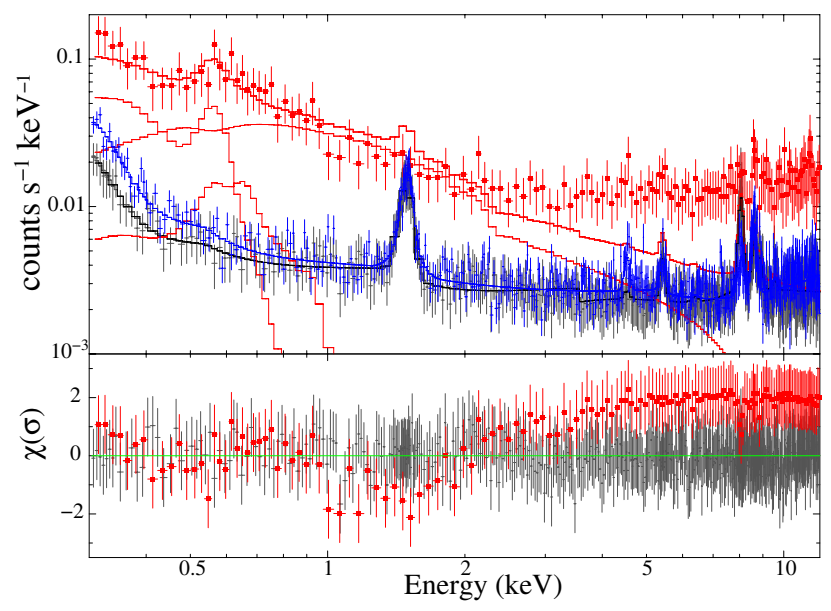

Fig. A.1. Example of a strong soft proton contamination (SPC) affecting the spectral analysis of MCSNR J0529-6653 (ObsID 0700381101). The instrumental background, extracted from FWC data, is shown in the top panel in grey and blue. The spectrum extracted from the BG region is shown in red. The strong flat tail above $2 \mathrm{keV}$ is the SPC, which the instrumental + AXB model cannot account for.

- Bad extraction region: if the SNR is very bright, a background spectrum extracted too close to the SNR will include contamination from the telescope PSF wings. Alternatively, a nearby bright X-ray source can produce artefacts such as singly reflected photons up to large angular distances $\left(\sim 1^{\circ}\right)$. When this happens, we selected the background in another region better suited.

- Anomalous MOS states: the model for the instrumental background of the MOS detectors was developed for data obtained in the normal state; Kuntz \& Snowden (2008) identified periods of "anomalous" background of EPIC-MOS. The instrumental background spectrum of this anomalous state is markedly stronger below $1 \mathrm{keV}$, which complicates the analysis of observations obtained during these epochs. If the SNR affected was faint, including the MOS data usually does not add much information, and only the pn spectrum was used. Conversely, if the SNR is bright, the error induced by fitting the anomalous spectra with the standard model is unimportant, because the source count rate is much higher than the instrumental background, and this issue can be discarded.

- Soft proton contamination: observations affected by a strong SPC are easily identified by fitting the FWC and astrophysical backgrounds spectra together. Indeed, the background above $2 \mathrm{keV}$ is almost purely instrumental. Therefore, a residual component at high energy in the background spectrum (extracted from science observation) that is not present in the FWC spectrum betrays the SPC. An extreme example is shown in Fig. A.1. An extra component needs to be added to the X-ray background and SNR models to account for the SPC.

\section{Appendix B: Selection of SNRs with secured classifications}

The list of LMC remants with secured CC and type Ia classifications is given in Table A.1.

\section{B.1. Type la SNRS}

The spectacular case of MCSNR J0509-6731: one of the few SNRs less than a thousand years old, this object was first typed as a type Ia remnant by Tuohy et al. (1982) based on the Balmerdominated optical spectrum. This classification was confirmed by the analysis of the ASCA spectrum, revealing ejecta emission rich in nucleosynthesis products of thermonuclear $\mathrm{SNe}$ (Hughes et al. 1995). Finally, light echoes from the SN, scattered off interstellar dust, were detected around four LMC SNRs (Rest et al. 2005). Optical spectroscopy of the light echoes of MCSNR J0509-6731 allowed Rest et al. (2008) to determine the SN spectral type as an overluminous 1991T-like SN Ia.

Balmer-dominated SNRs with X-ray-detected ejecta: Tuohy et al. (1982) also detected Balmer-dominated emission from MCSNR J0519-6902, J0505-6753 (DEM L71), and J0547-7025, concluding that they were produced by type Ia events. In the two former cases, the X-ray spectra clearly showed emission from the ejecta of thermonuclear SNe (Hughes et al. 1995, 2003; van der Heyden et al. 2003). For J0547-7025, the Chandra spectra revealed ejecta but the $\mathrm{O} / \mathrm{Fe}$ ratio was not as decisive (Hendrick et al. 2003). Furthermore, this remnant was an outlier in Lopez et al. (2009, 2011), with a morphology more consistent with the sample of CC-SNRs. The observations of the XMM-Newton survey confirm the iron-rich nature of J0547-7025 (Table E.1), and therefore secure a type Ia classification consistent with the optical data.

Middle-aged to evolved iron-rich SNRs: several remnants with ages exceeding $10^{4}$ yr revealed iron-rich X-ray spectra (observed with XMM-Newton and Chandra) that betrayed their type Ia nature. MCSNR J0534-6955 was first identified as such with Chandra (Hendrick et al. 2003) and XMM-Newton observations give similar results (Table E.1). Slightly more evolved, MCSNR J0534-7033 and J0536-7039 (DEM L238 and L249, respectively) have a more pronounced separation of the shell and central iron-rich plasma (Borkowski et al. 2006). The shell A of DEM L316 (MCSNR J0547-6941) has striking spectral differences to the very close neighbour MCSNR J0547-6943 (DEM L316B): the former is also mostly exhibiting Fe L-shell emission, which leads to the interpretation that it is another type Ia remnant (Nishiuchi et al. 2001; Williams \& Chu 2005).

Then come three more evolved (age $\gtrsim 15 \mathrm{kyr}$ ) iron-rich SNRs that were presented in Maggi et al. (2014) and Bozzetto et al. (2014). Finally, since these publications, we obtained $X M M-N e w t o n$ follow-up observations of two remnants, which we classified as type Ia: i) MCSNR J0506-7026 was a ROSATselected candidate ([HP99] 1139), which revealed a remnant similar to DEM L238 and L249, about 17-21 kyr old and holding about $0.9 M_{\odot}-1 M_{\odot}$ in the central region (Kavanagh et al., in prep., Table E.1); ii) MCSNR J0527-7104 was confirmed by our group in a multi-wavelength study (Kavanagh et al. 2013). A subsequent observation (performed 2014 May 31) revealed yet another iron-rich core (with an unusual morphology, see Kavanagh et al., in prep.), so that this source completes the (currently known) sample of LMC SNRs with a secured type Ia origin.

\section{B.2. Core-collapse SNRs}

Remnants hosting a compact object: several neutron stars have been detected inside LMC SNRs, mostly powering a pulsar wind nebula (PWN). MCSNR J0540-6920 is the prototypical example: It hosts the pulsar PSR B0540-69 and is known as a twin of the Crab nebula (Kaaret et al. 2001). MCSNR J0537-6910 (N157B) is also dominated by a PWN around PSR J0537-6910 (Chen et al. 2006). Fainter, less obvious PWNe 
Table A.1. LMC remnants with a secured SN classification.

\begin{tabular}{|c|c|c|c|c|}
\hline MCSNR & Other name & $\begin{array}{l}\text { Age } \\
(\mathrm{yr})\end{array}$ & Evidence $^{a}$ & References $^{b}$ \\
\hline \multicolumn{5}{|c|}{ Core-collapse SNRs } \\
\hline J0536-6916 & SNR 1987A & 28 & Historical & Maggi et al. (2012b) \\
\hline J0540-6920 & B0540-693 & $\sim 1600$ & Pulsar & Kaaret et al. (2001) \\
\hline J0525-6938 & N132D & $\sim 3150$ & Ejecta, morphology & Borkowski et al. (2007) \\
\hline J0535-6602 & N63A & $2000-5000$ & Ejecta, Fe K & Warren et al. (2003) \\
\hline J0536-6913 & - & $2200-4900$ & Ejecta & Kavanagh et al. (2015a) \\
\hline J0505-6802 & $\mathrm{N} 23$ & $\sim 4600$ & Ejecta, CCO, morphology & Hughes et al. (2006) \\
\hline J0526-6605 & N49 & $\sim 4800$ & Ejecta, SGR, Fe K & Park et al. (2012) \\
\hline J0537-6910 & N157B & $\sim 5000^{c}$ & PWN & Chen et al. (2006) \\
\hline J0525-6559 & N49B & $\sim 10000$ & Ejecta, morphology & Park et al. (2003b) \\
\hline J0536-6735 & DEM L241 & $>10^{4 c}$ & HMXB & Seward et al. (2012) \\
\hline J0453-6829 & B0453-685 & $12000-15000$ & PWN, morphology & Haberl et al. (2012a) \\
\hline J0531-7100 & $\mathrm{N} 206$ & $\sim 25000$ & PWN candidate, morphology & Williams et al. (2005) \\
\hline \multicolumn{5}{|c|}{ Type Ia SNRs } \\
\hline J0509-6731 & B0509-675 & $400 \pm 120$ & $\begin{array}{l}\text { Light echo, ejecta } \\
\text { Fe K, morphology }\end{array}$ & Rest et al. (2008) \\
\hline J0509-6844 & N103B & 860 & Ejecta, Fe K & Hughes et al. (1995) \\
\hline J0519-6902 & B0519-690 & $600 \pm 200$ & Ejecta, Fe K, morphology & Hughes et al. (1995) \\
\hline J0505-6753 & DEM L71 & $\sim 4700$ & Ejecta. Fe K, morphology & Hughes et al. $(1998,2003)$ \\
\hline J0547-7025 & B0548-704 & $\sim 7100$ & Ejecta & Hendrick et al. (2003), this work \\
\hline J0534-6955 & B0534-699 & $\sim 10000$ & Ejecta, morphology & Hendrick et al. (2003) \\
\hline J0534-7033 & DEM L238 & $\sim 13500$ & Ejecta & Borkowski et al. (2006) \\
\hline J0536-7039 & DEM L249 & $\sim 15000^{c}$ & Ejecta & Borkowski et al. (2006) \\
\hline J0506-7026 & [HP99] 1139 & $17000-21000$ & Ejecta & Kavanagh et al. (in prep.), this work \\
\hline J0508-6902 & [HP99] 791 & $20000-25000$ & Ejecta & Bozzetto et al. (2014) \\
\hline J0527-7104 & [HP99] 1234 & $\sim 25000$ & Ejecta & Kavanagh et al. (2013), this work \\
\hline J0547-6941 & DEM L316A & $\sim 27000^{c}$ & Ejecta & Williams \& Chu (2005) \\
\hline J0511-6759 & - & $\gtrsim 20000^{c}$ & Ejecta & Maggi et al. (2014) \\
\hline J0508-6830 & - & $\gtrsim 20000^{c}$ & Ejecta & Maggi et al. (2014) \\
\hline
\end{tabular}

Notes. Ages for the first three type Ia SNRs are from light echo measurements (Rest et al. 2005). ${ }^{(a)}$ Morphology: Typed from X-ray morphology by Lopez et al. (2009, 2011). Fe K: Typed from the properties of the Fe K emission by Yamaguchi et al. (2014, see also Sect. 5.4). CCO: Central compact object. PWN: Pulsar wind nebula. SGR: Soft gamma-ray repeater. ${ }^{(b)}$ Because of the multiple studies on most remnants, the given references are "see $[\ldots]$ and references therein". ${ }^{(c)}$ Uncertain age.

have been found in MCSNR J0453-6829 (Gaensler et al. 2003; Haberl et al. 2012a) and in MCSNR J0535-6602 (Williams et al. 2005). In the latter, the case for a PWN is not as strong; however, analysis of the X-ray morphology using a power-ratio method (Lopez et al. 2009) confirm the classification as CC-SNR.

Chandra observations (Hughes et al. 2006) of MCSNR J0505-6802 (N23) revealed, in addition to regions with enhanced O-group elements, a point source in the centre of the remnant that shows properties similar to compact central objects (CCOs) seen in other CC-SNRs, such as Cas A. Finally, a point source was detected in the "Head" of MCSNR J0536-6735 (DEM L241, see Bamba et al. 2006) using XMM-Newton observations and first classified as candidate PWN. With Chandra observations, Seward et al. (2012) could show that the source was not extended and identified the optical counterpart as an O5III(f) star. Based on this and the X-ray variability and spectrum, they concluded that the SNR was hosting an HMXB, akin to SXP1062 in the SMC (Hénault-Brunet et al. 2012).

Detection of the remains of massive star nucleosynthesis: MCSNR J0525-6938 (N132D), the brightest SNR in the LMC, belongs to the class of oxygen-rich remnants. Many clumps of X-ray emitting O-ejecta are detected in X-ray observations (Borkowski et al. 2007) and match the optical ejecta morphology seen by Hubble. Park et al. (2003b) have revealed a highly enhanced $\mathrm{Mg}$ abundance and derived a high mass of $\mathrm{Mg}$ ejecta from MCSNR J0525-6559 (N49B), strongly suggesting a massive stellar progenitor. This classification is supported by the X-ray morphology of the remnant (Lopez et al. 2009).

The nearby MCSNR J0526-6605 (N49) is a more puzzling case. No compelling evidence for overabundant $\mathrm{O}$ or $\mathrm{Fe}$ is found, but $\mathrm{Si}$ - and $\mathrm{S}$-rich ejecta features are detected by Chandra (Park et al. 2003a, 2012). These can be interpreted as explosive O-burning or incomplete Si-burning deep inside a CC SN explosion; however, the $\mathrm{Si} / \mathrm{S}$ ejecta mass ratio favour a type Ia origin (Park et al. 2003a, 2012). The soft gamma-ray repeater SGR 0526-66 lies in projection in the remnant, favouring the CC-SNR scenario, although the physical association between the remnant and the SGR is uncertain (Gaensler et al. 2001; Kaplan et al. 2001). The best evidence to terminate the debate over the nature of N49 comes from the properties of its Fe K emission, which is clearly in the region occupied by CC-SNRs (Yamaguchi et al. 2014, Sect. 5.4). MCSNR J0535-6916 (N63A) is another case where ejecta features are detected but cannot yield a definite classification (Warren et al. 2003). As for N49, however, the Fe K emission allows N63A to be included in the sample of secured CC-SNRs.

SNR 1987A: last but not least comes the remnant with the most secured core-collapse classification of all, as the SN itself was observed in detail and its progenitor identified in pre-explosion images. 
P. Maggi et al.: The population of X-ray supernova remnants in the Large Magellanic Cloud

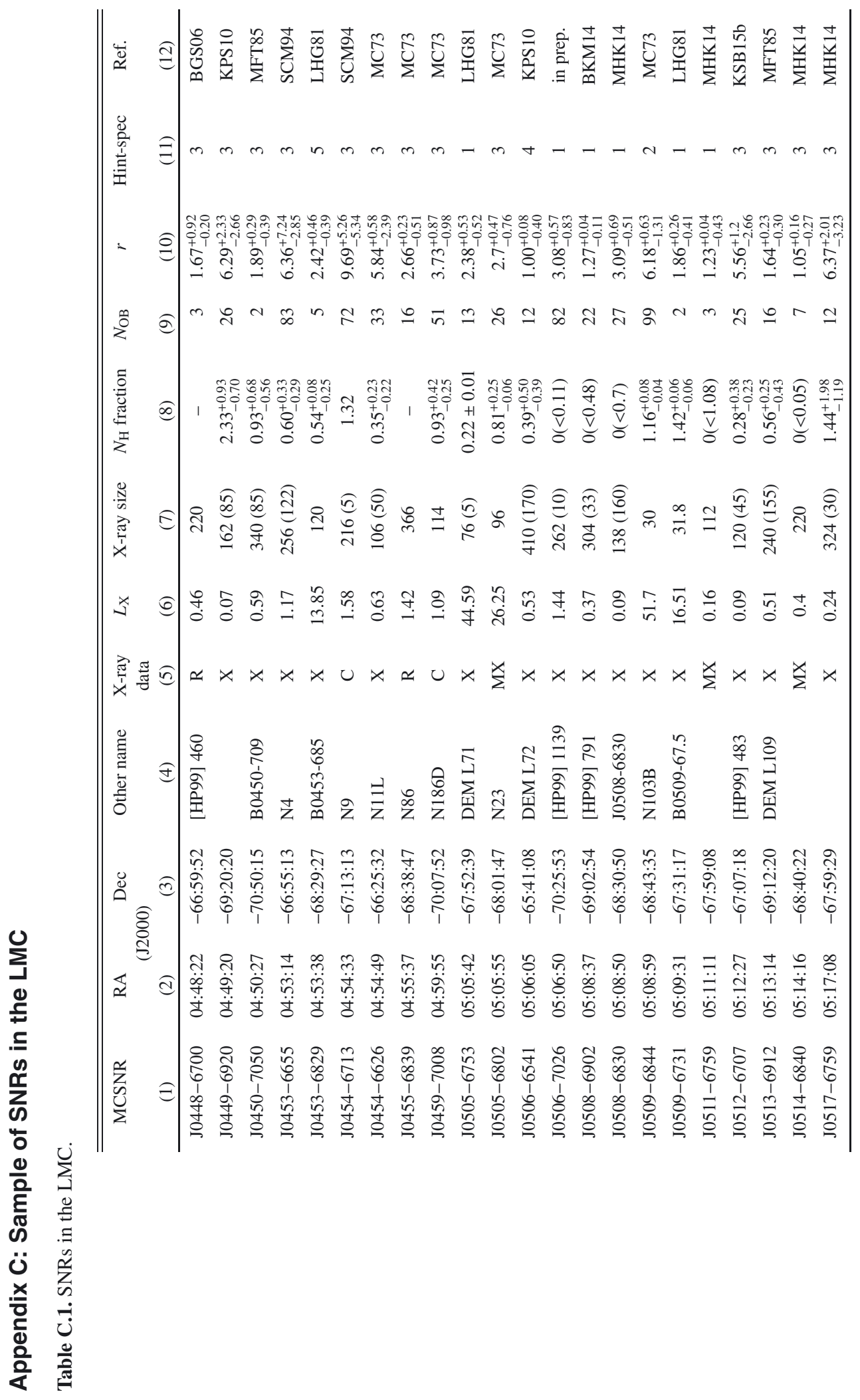

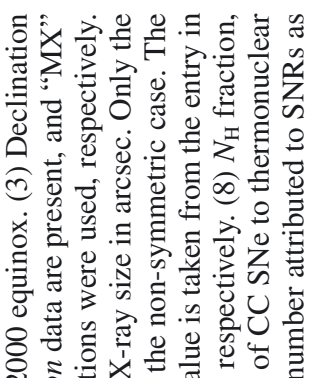

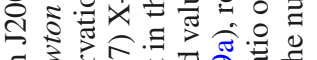

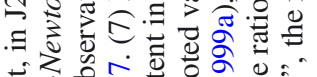

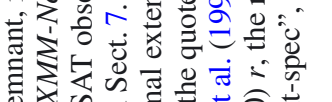

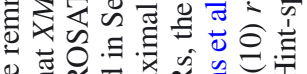

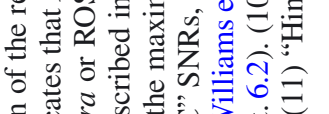

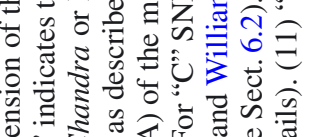

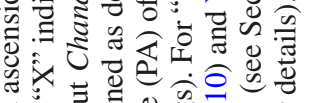

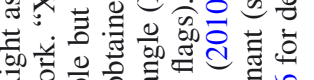

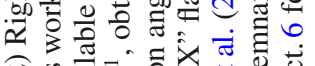

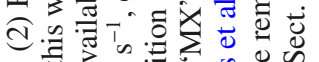

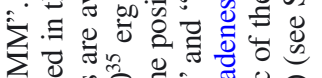

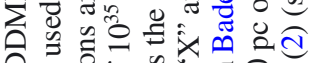

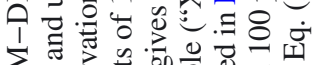

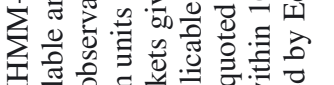

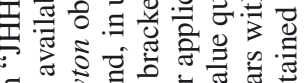

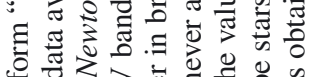

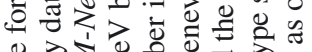

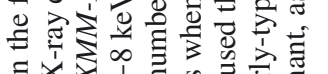

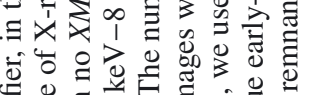

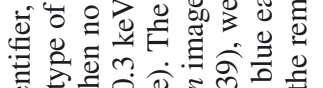

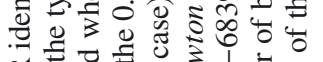

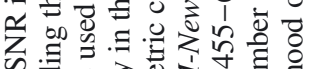

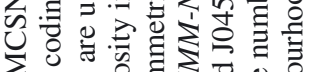

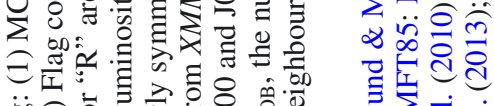

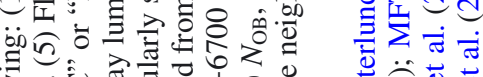

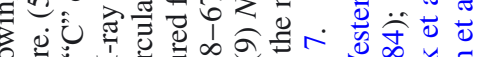

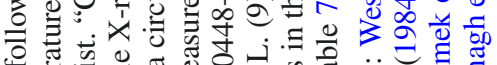

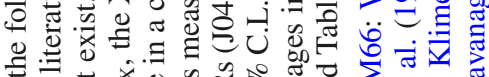

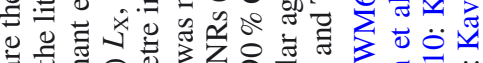

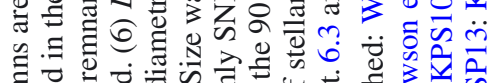

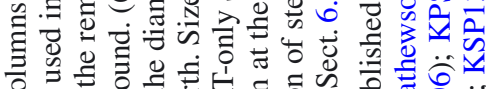

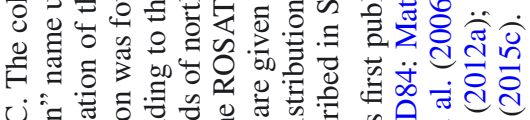

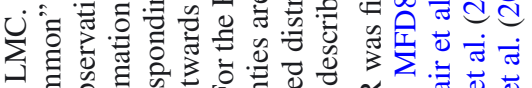

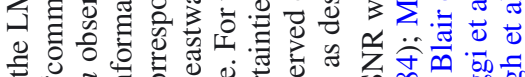

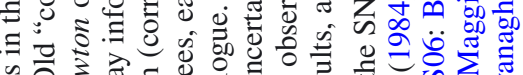

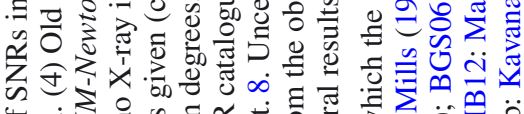

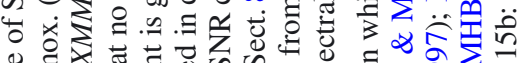

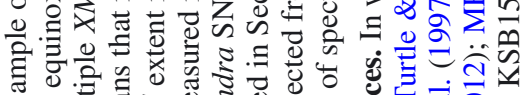

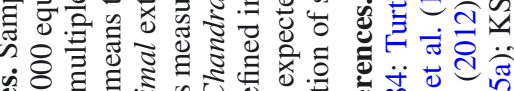

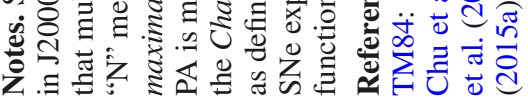


A\&A 585, A162 (2016)

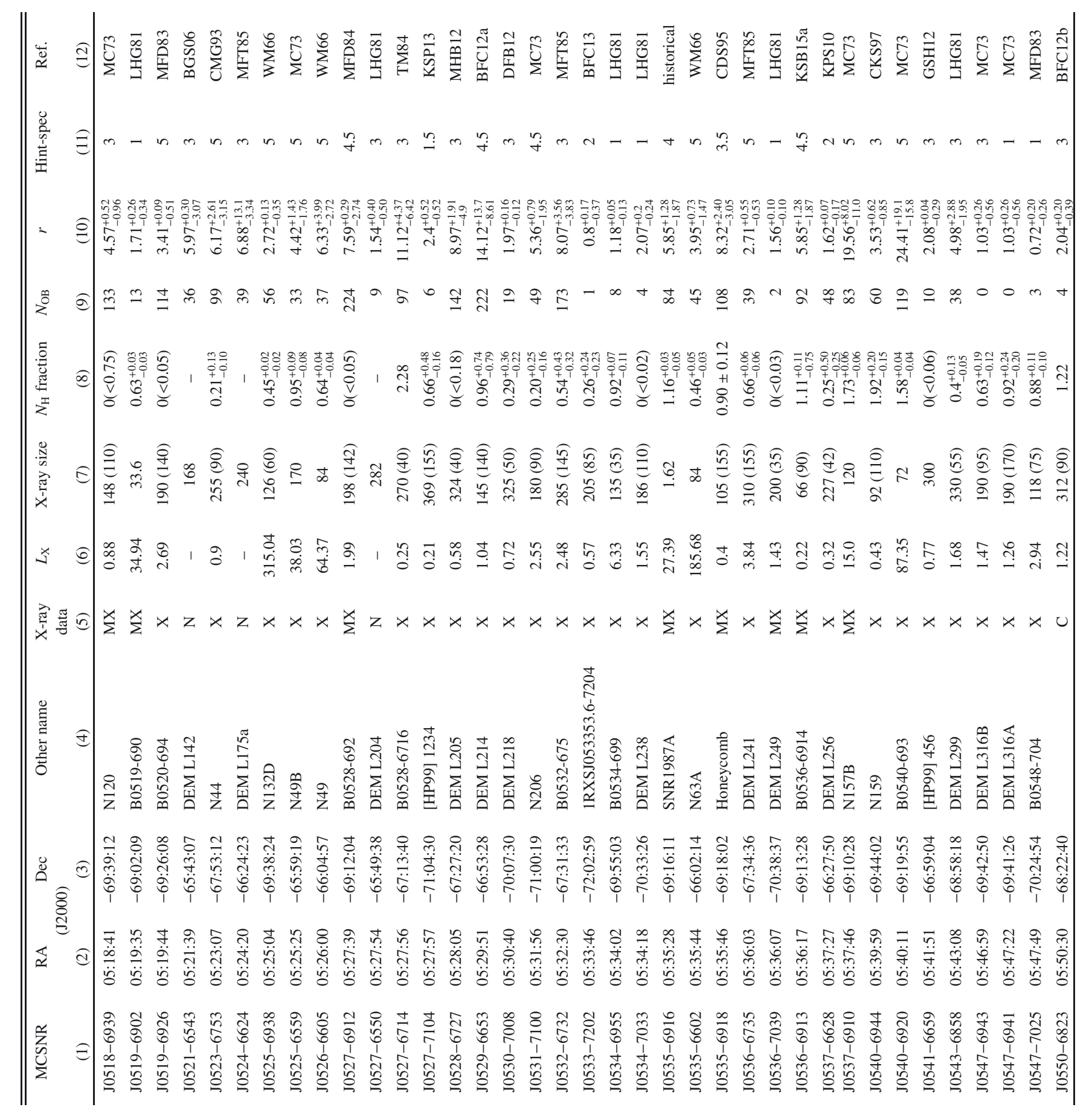

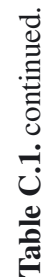


P. Maggi et al.: The population of X-ray supernova remnants in the Large Magellanic Cloud

\section{Appendix D: Spectra of SNRs observed with XMM-Newton for the first time}
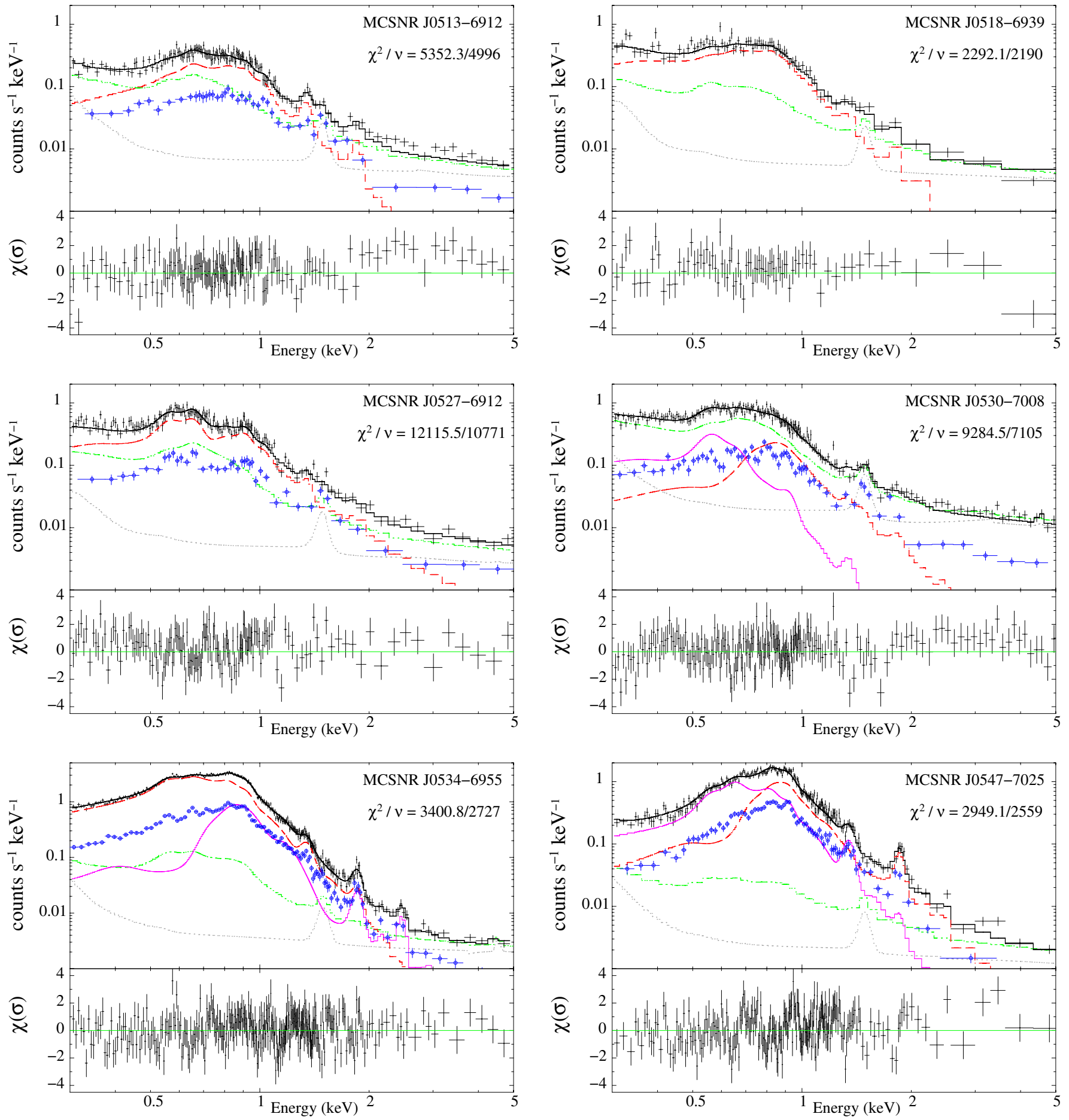

Fig. D.1. XMM-Newton EPIC spectrum of SNRs for which none have been published yet (see Sect. 5.1). The SNR names are labelled on each plot. The pn and MOS1 data are shown in black and blue points, respectively. MOS2 data are not shown for clarity. The total best-fit model (see parameters in Table E.1), convolved with the pn response, is shown as the solid black line. It includes: i) the instrumental background component with a dotted grey line (only pn shown); ii) the total AXB model (see Appendix A) with a dot-dashed green line; and iii) the SNR emission with a dashed red line. When a second component is used (Table E.1), it is shown with a solid magenta line. The lower panels show the pn residuals (in terms of $\sigma$ ) for the total best-fit model. For MCSNR J0527-6712 data from two observations were used, though only one spectra is shown for clarity. 


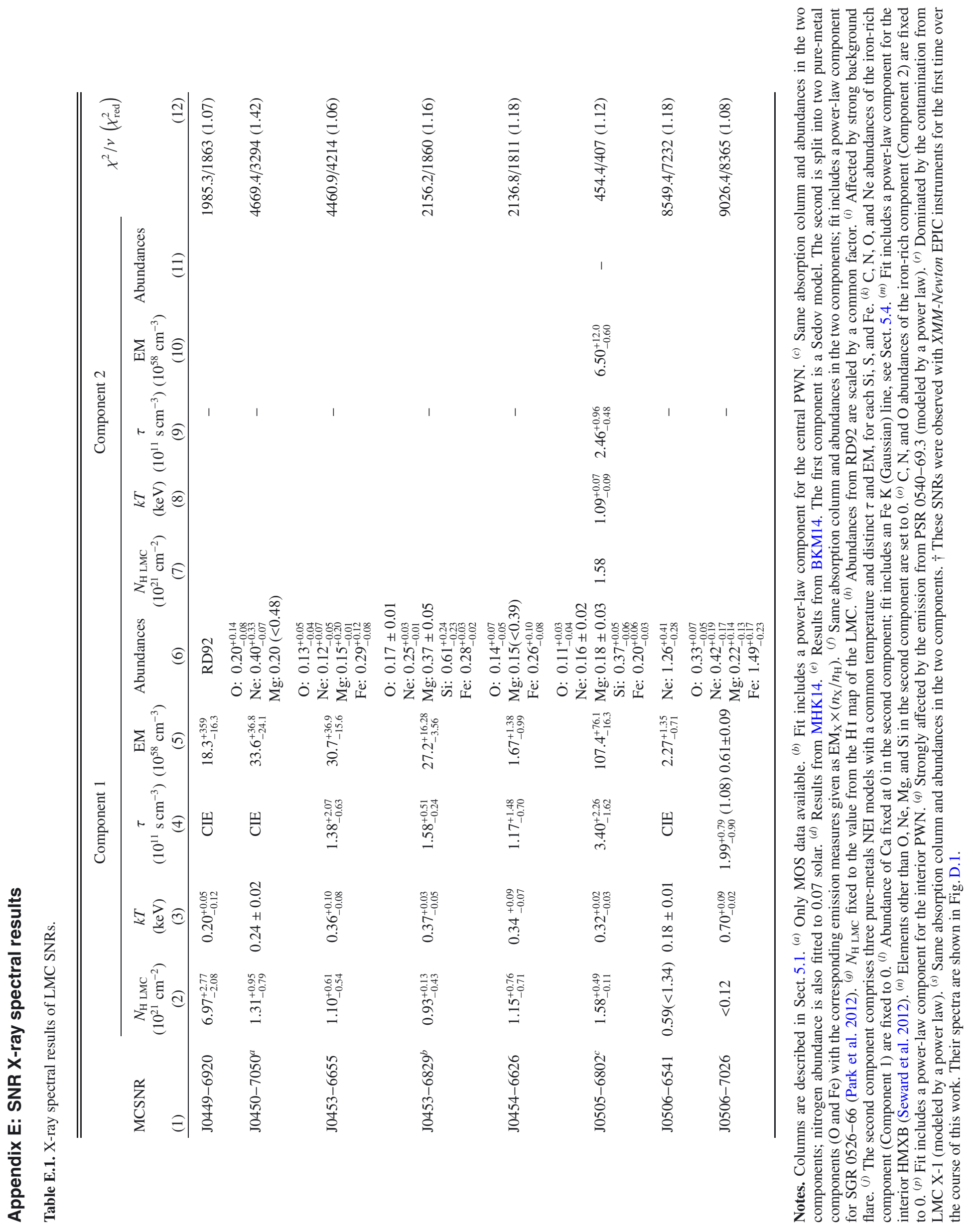




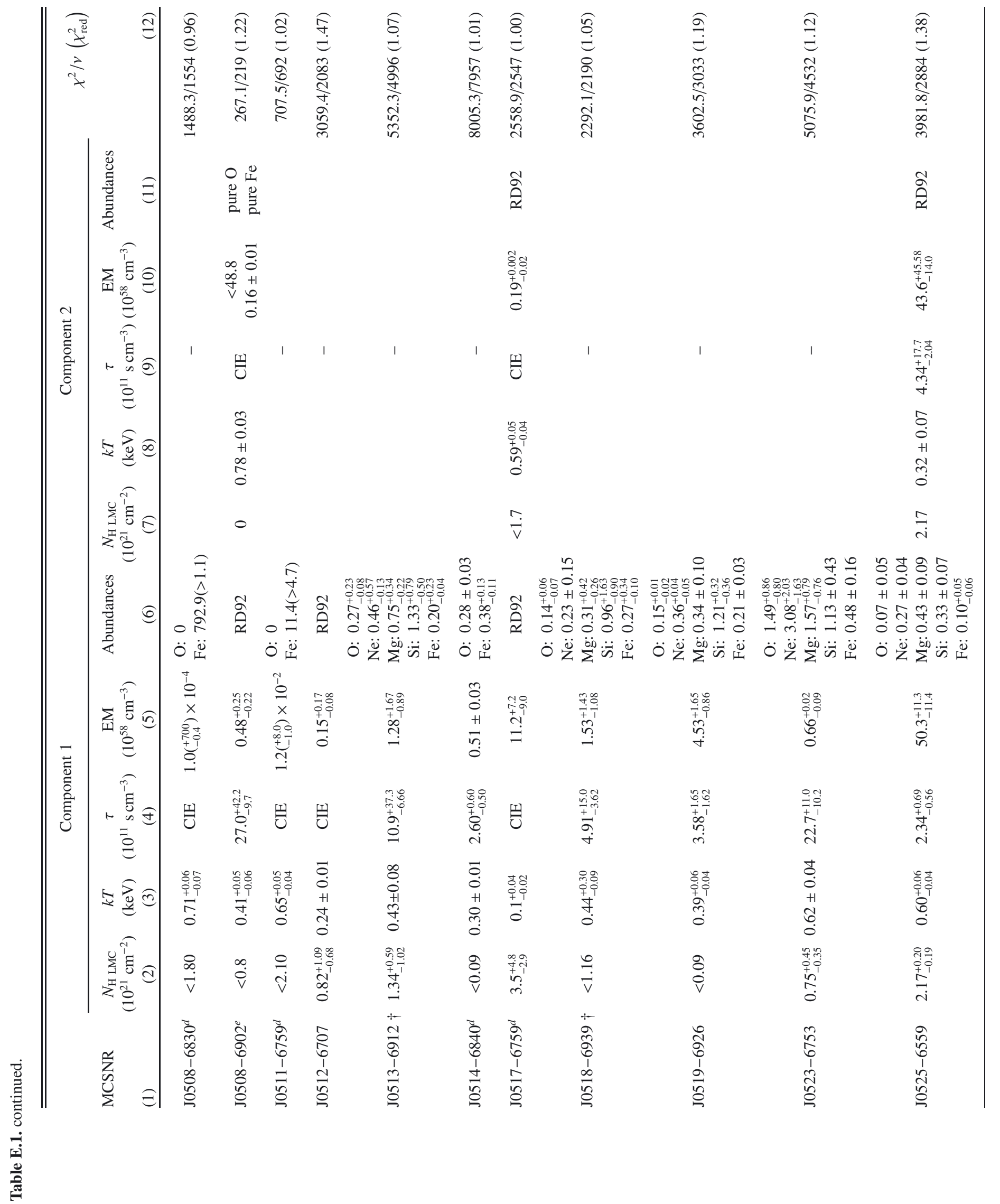




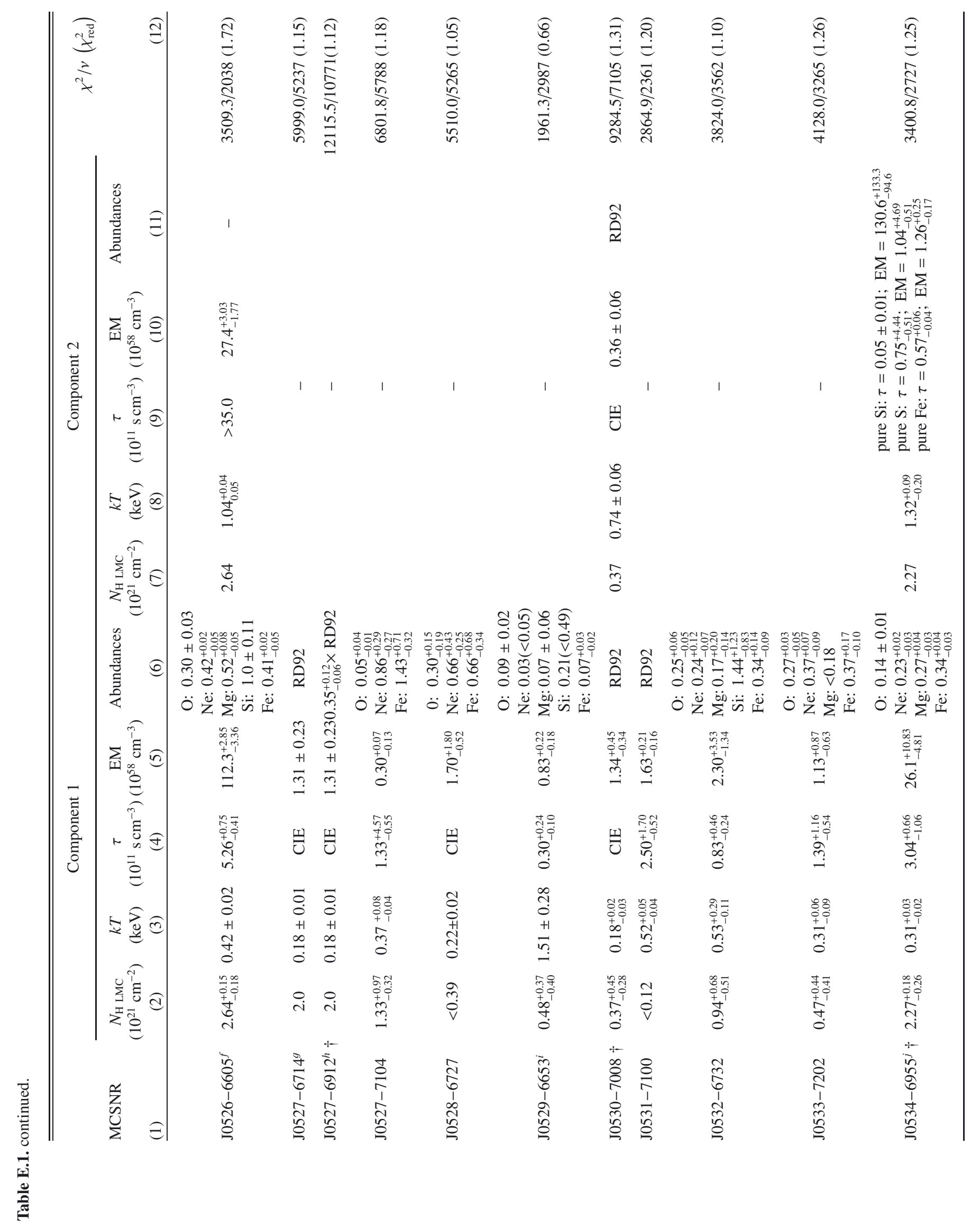




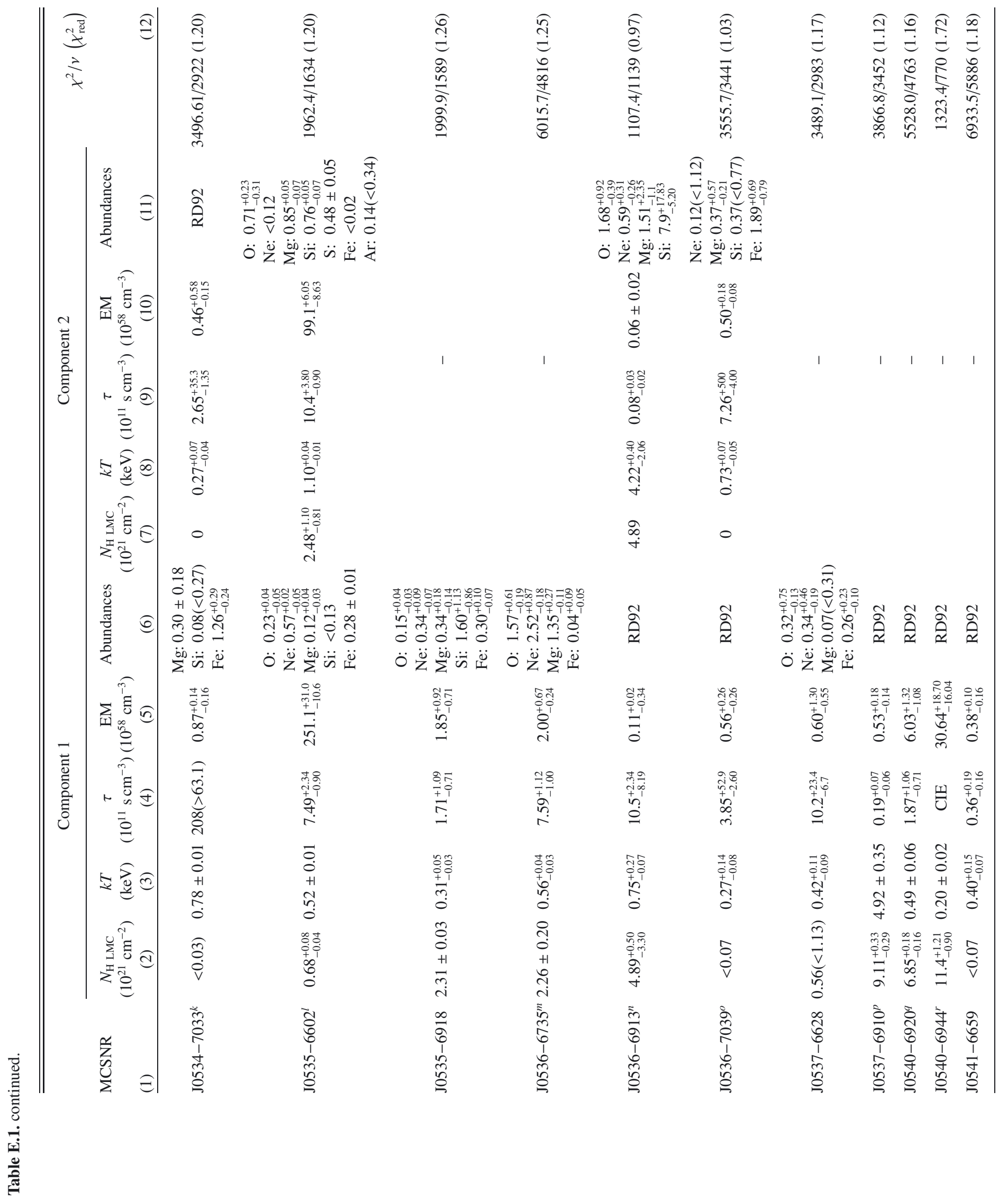




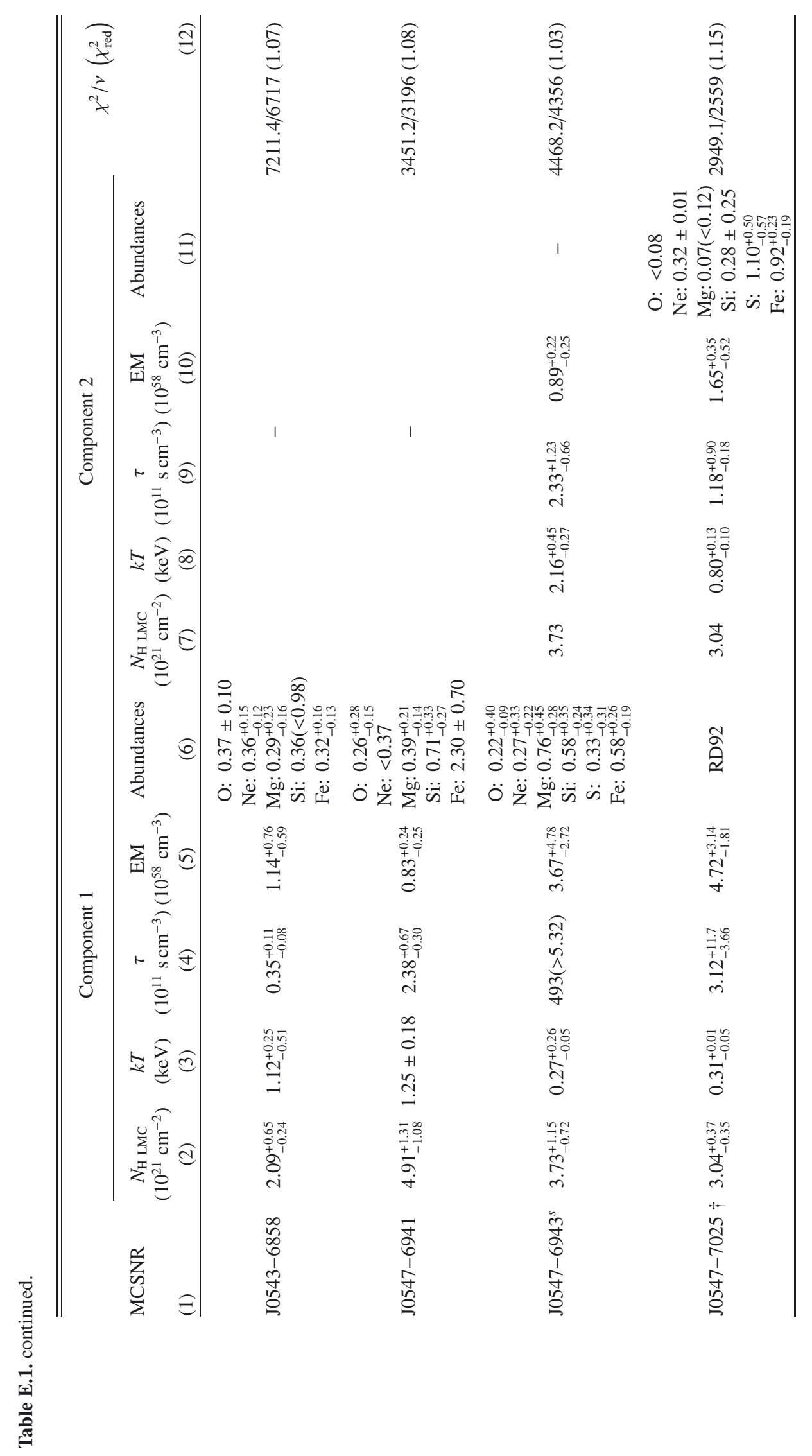


P. Maggi et al.: The population of X-ray supernova remnants in the Large Magellanic Cloud

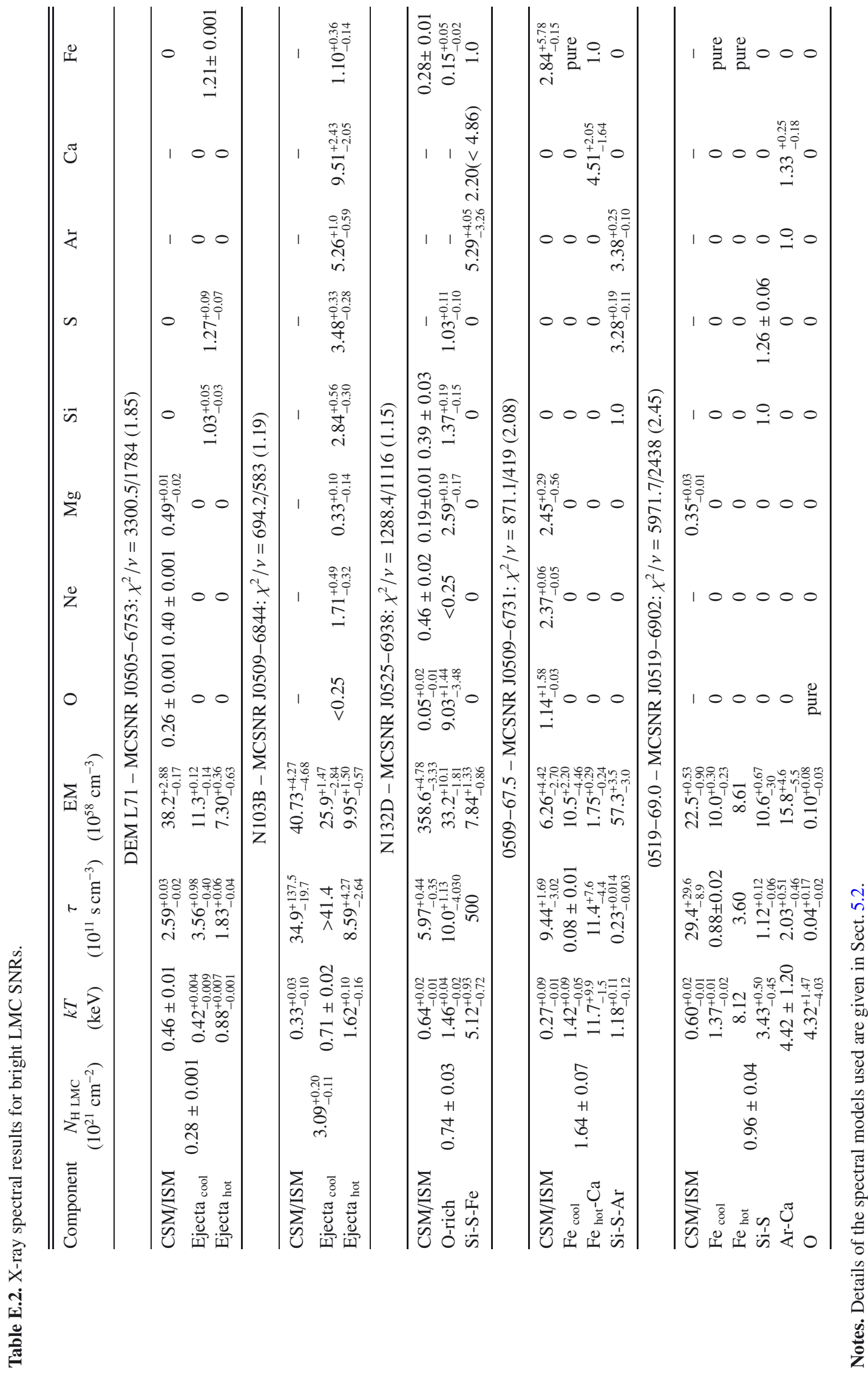




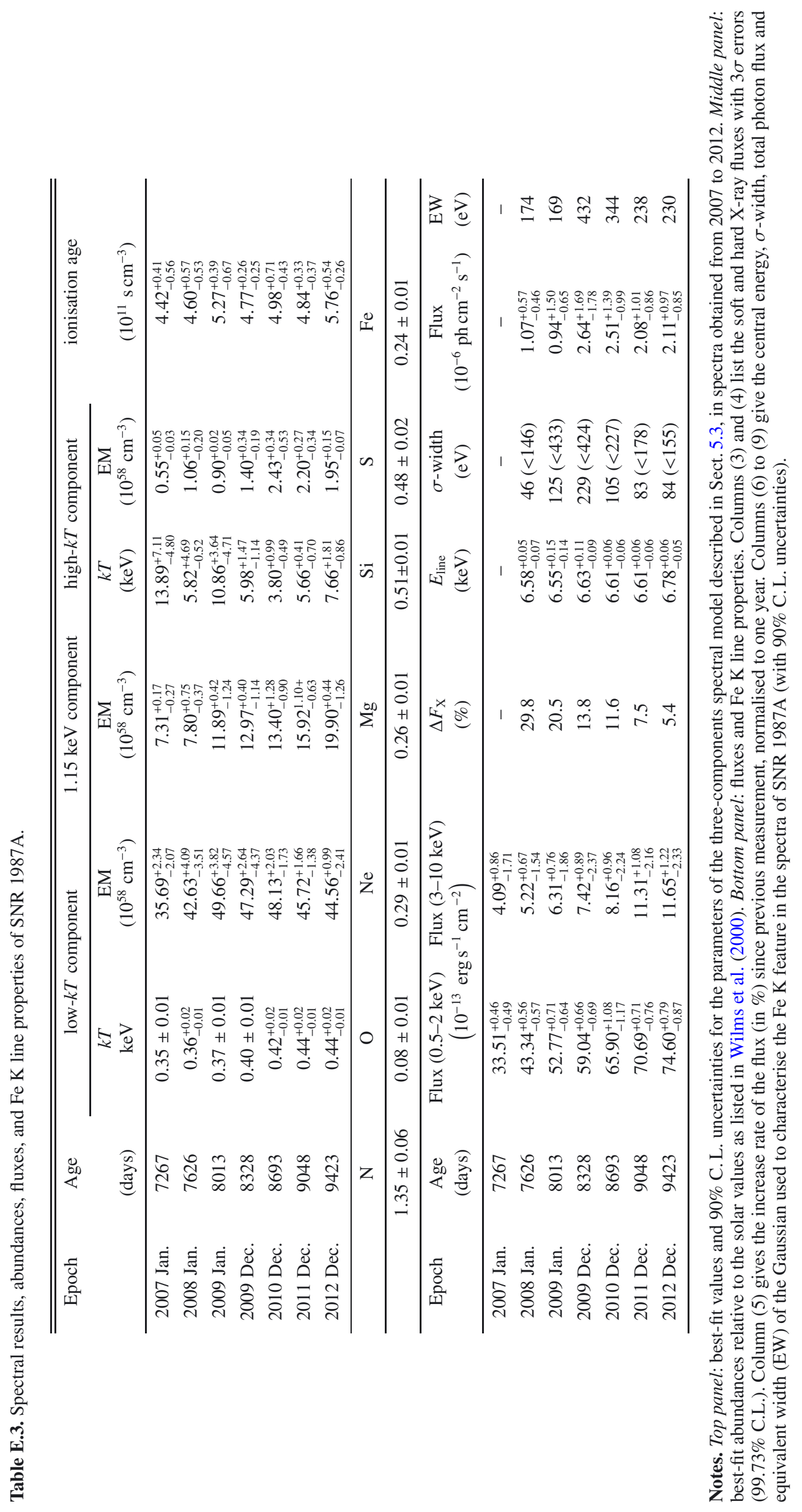




\section{Appendix F: X-ray images, spectral extraction regions, and SFH for all LMC SNRs}

This Appendix presents, for each SNR, an X-ray image (top), the regions used for spectral analysis (middle), and the SFH of the cell including the remnant (bottom panel). The images are using the $(0.3-0.7 \mathrm{keV})$, medium $(0.7-1.1 \mathrm{keV})$, and hard $(1.1-4.2 \mathrm{keV})$ bands as red, green, and blue components, respectively, and have been adaptively smoothed (see Sect. 2.2. The white bars indicate the scale of $1^{\prime}$. North is up and east is left. A linear scale is used to display the pixel values, but the cut levels are adapted for each SNR. The extraction regions used for spectral analysis are shown for pn, MOS1, and MOS2 detectors (left to right), as in Fig. 3. The star formation history plots are shown as in Fig. 8. For MCSNR J0509-6844, J0509-6731, and J0509-6902, which are highly unresolved with XMM-Newton, we show Chandra images, using processed data obtained from the Chandra SNR catalogue.

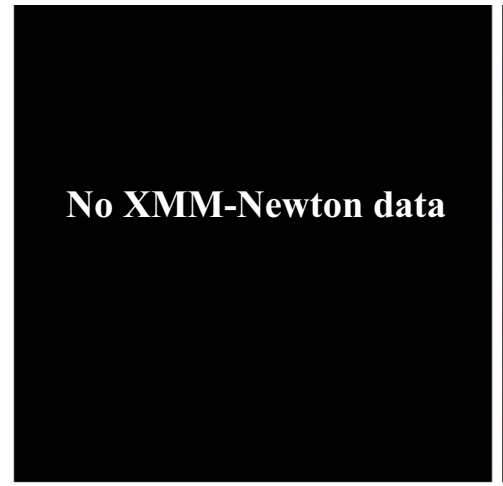

No XMM-Newton spectra
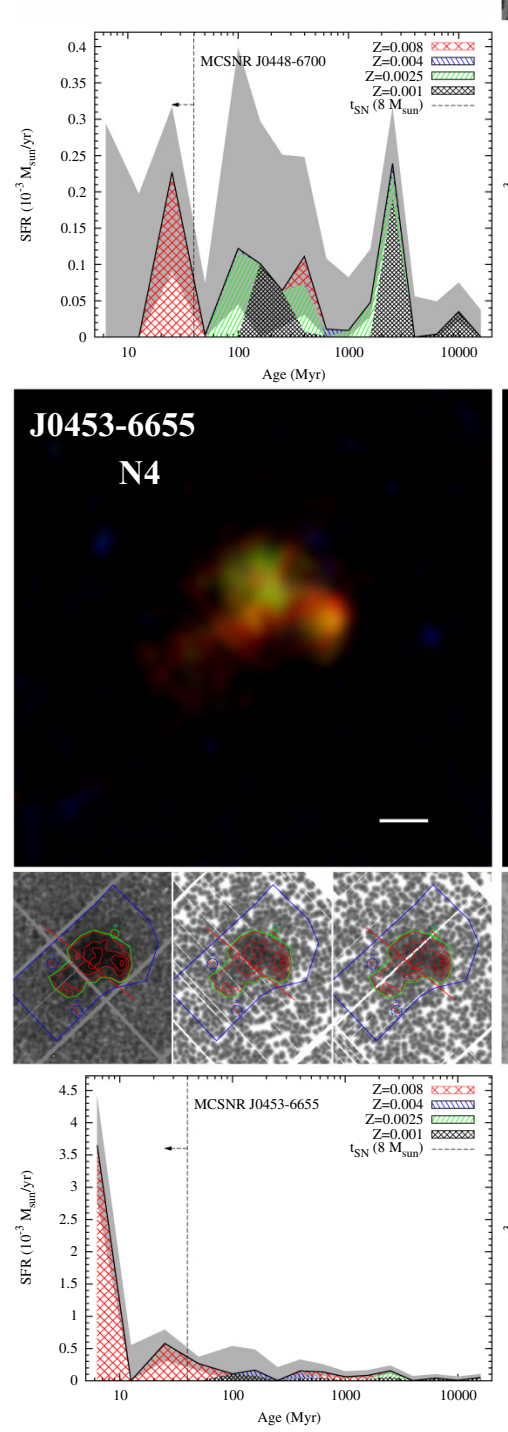
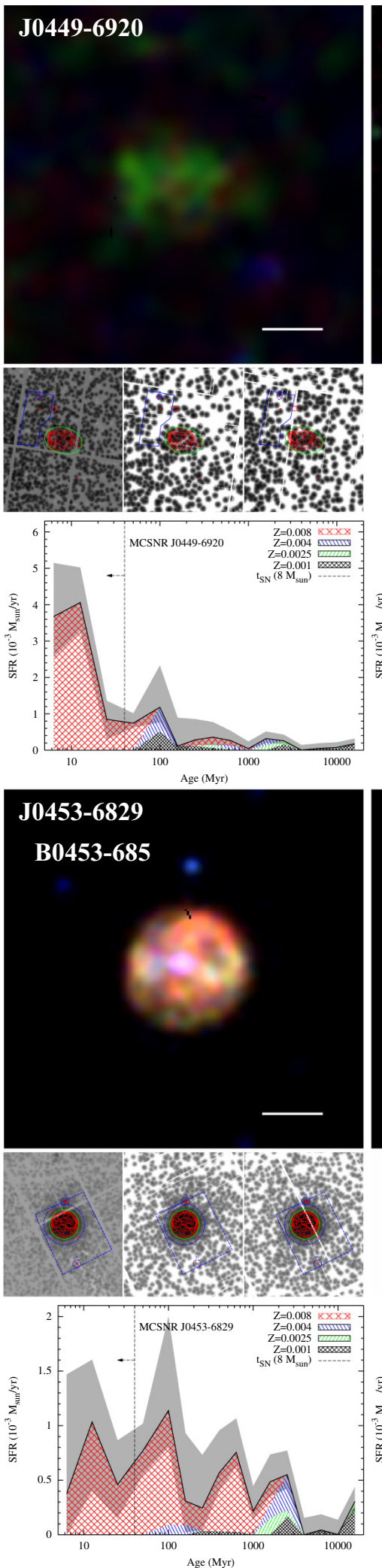
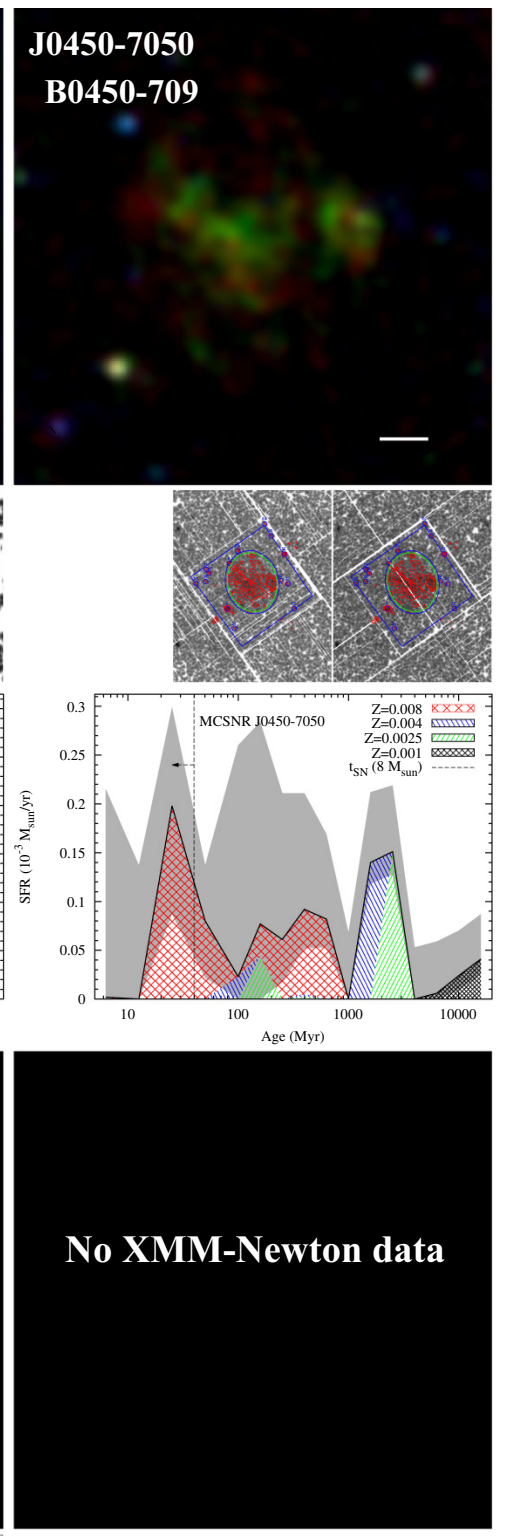

No XMM-Newton spectra

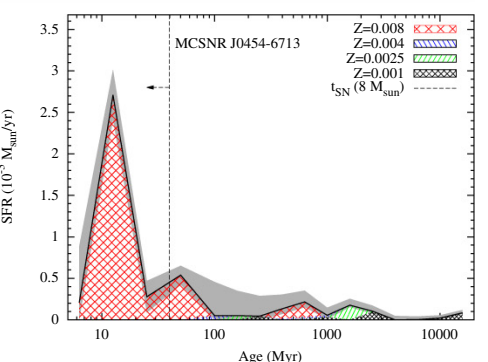



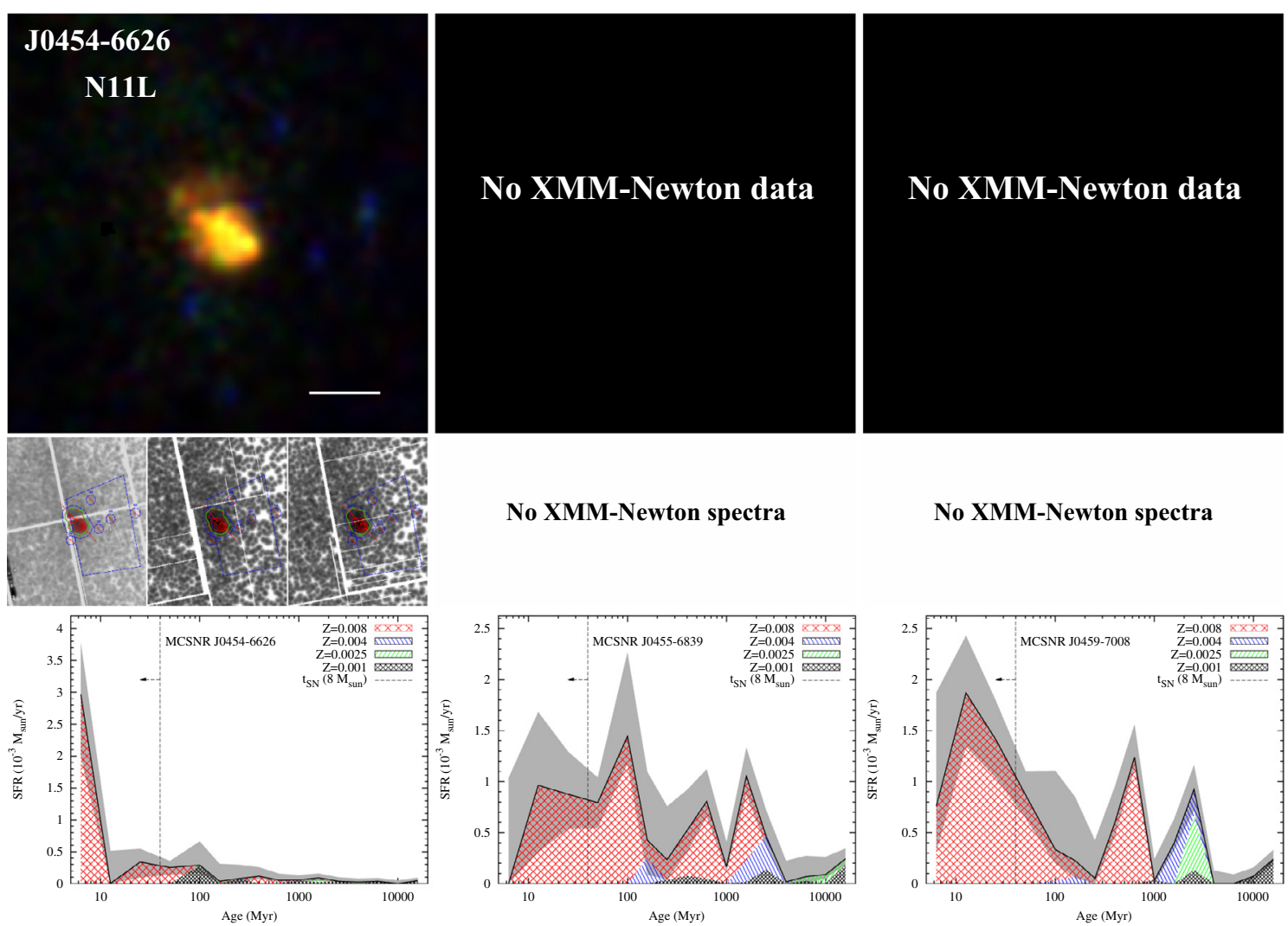

No XMM-Newton spectra

No XMM-Newton spectra
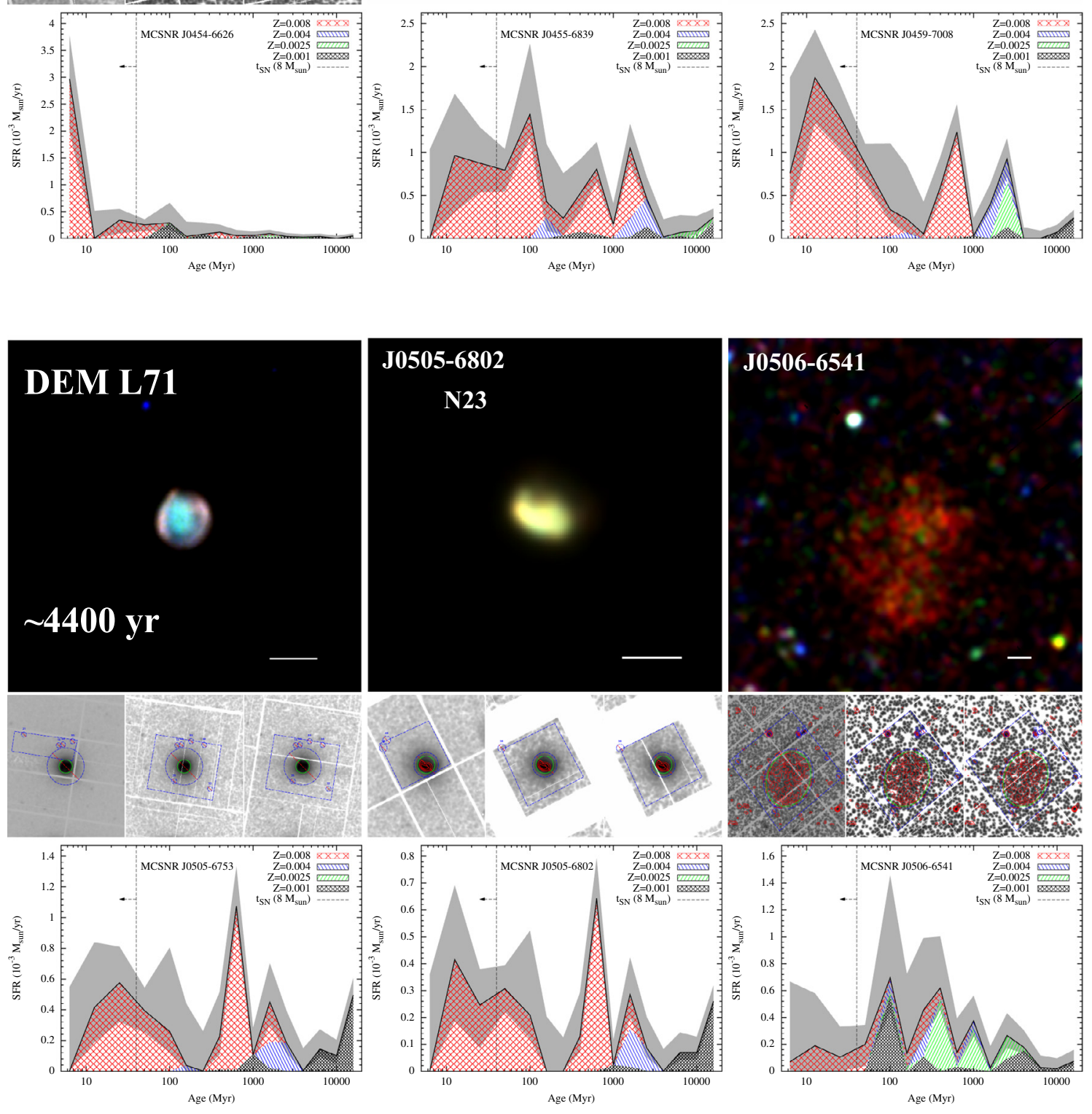
P. Maggi et al.: The population of X-ray supernova remnants in the Large Magellanic Cloud
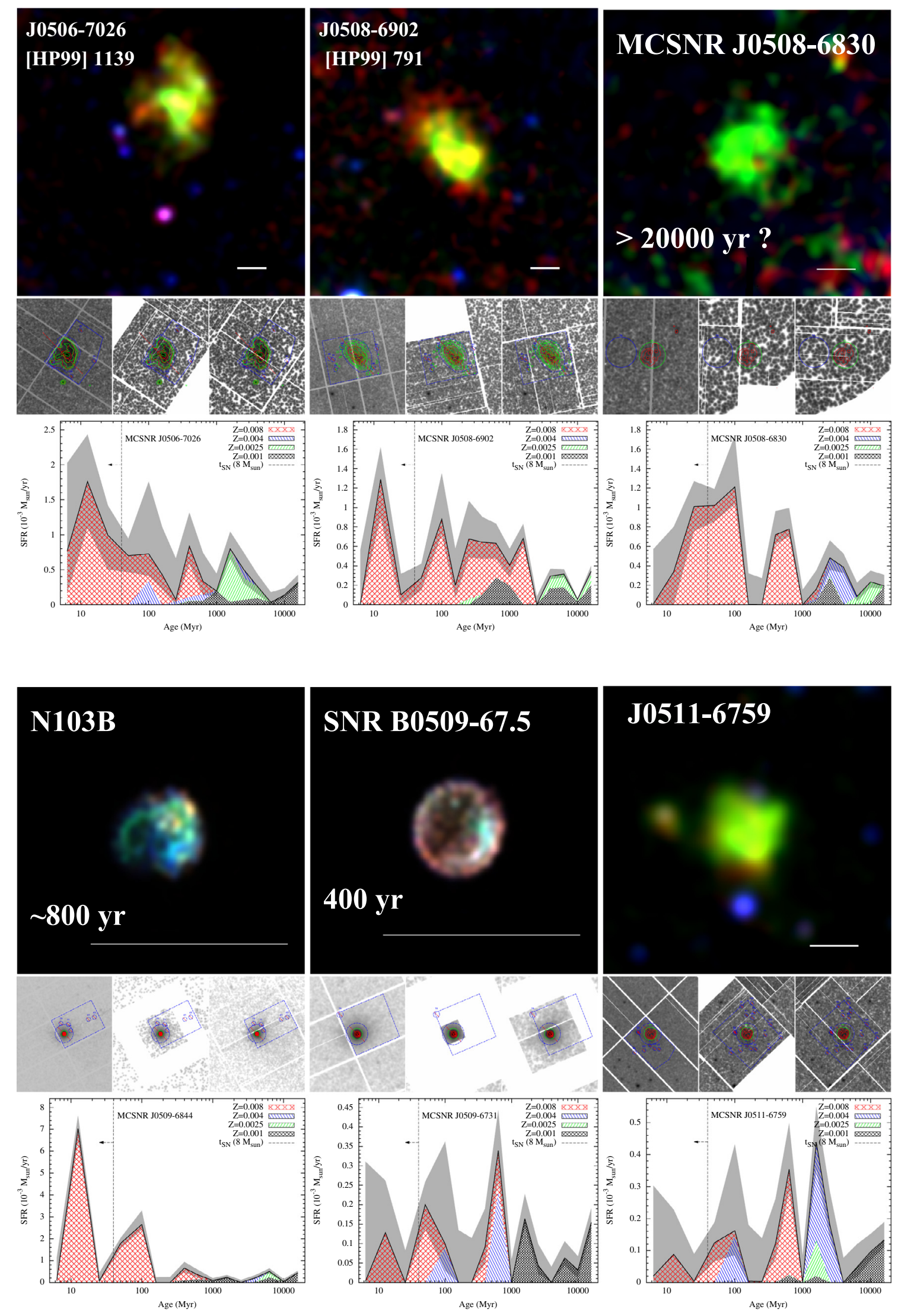

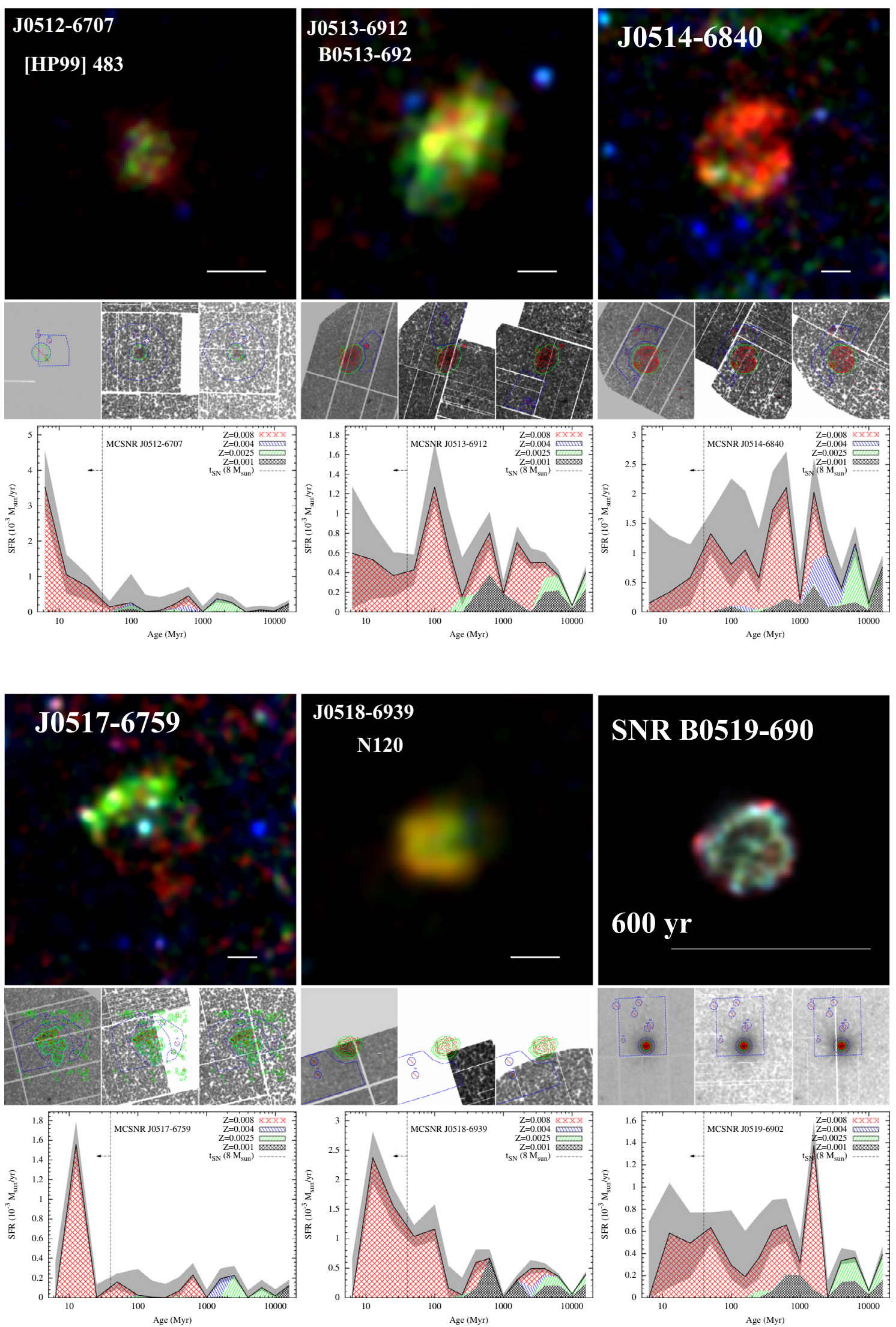
P. Maggi et al.: The population of X-ray supernova remnants in the Large Magellanic Cloud
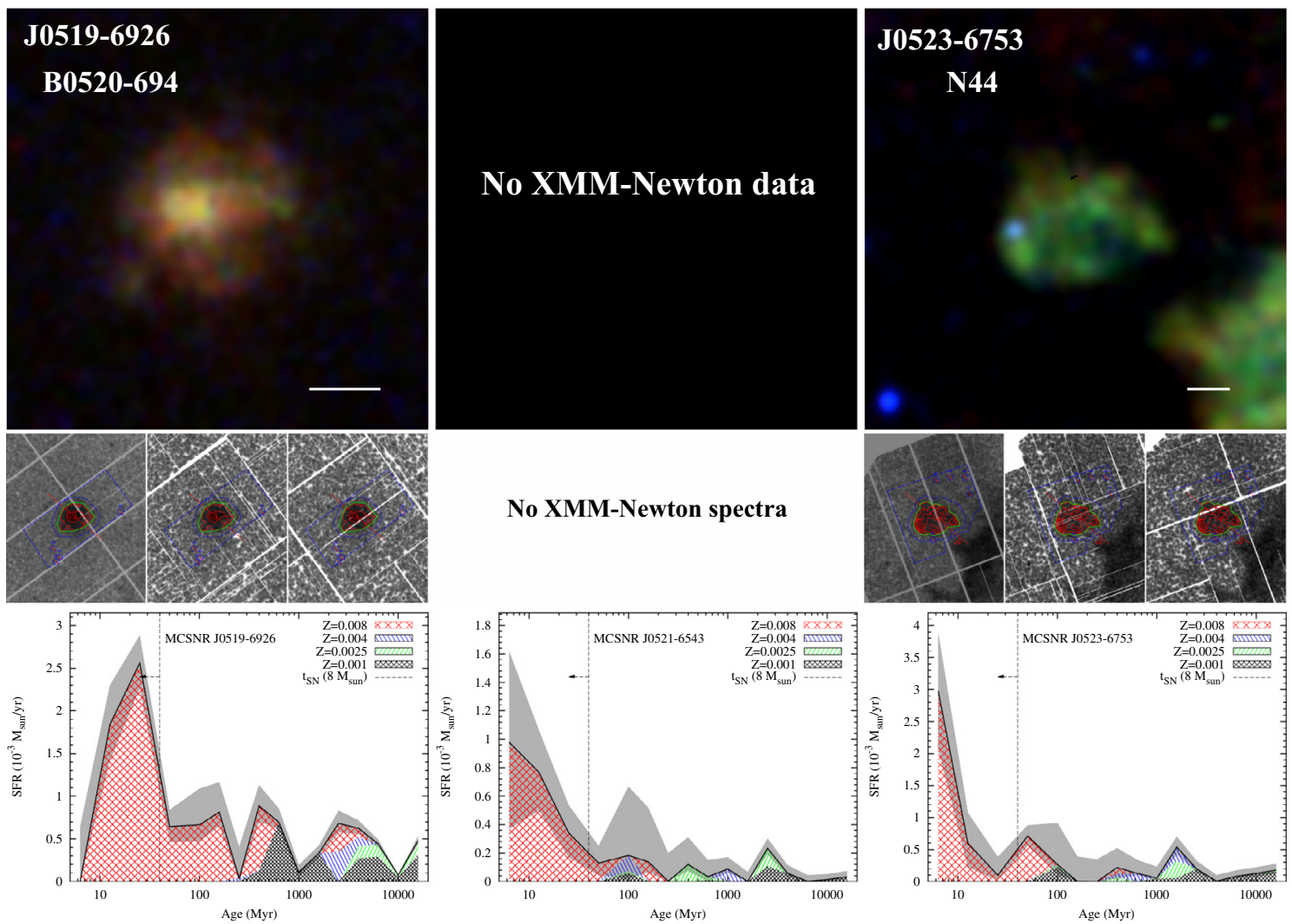

No XMM-Newton spectra
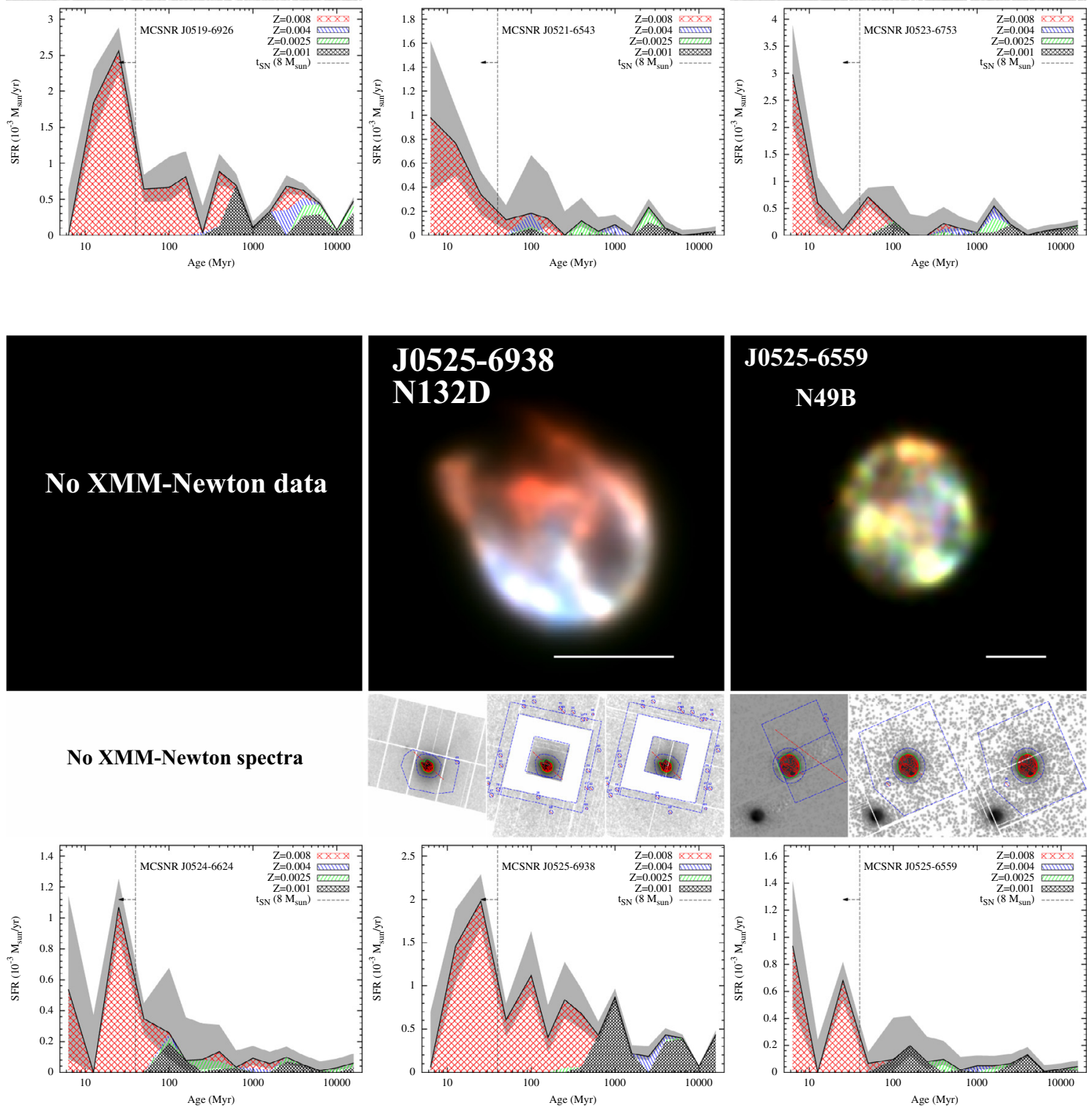

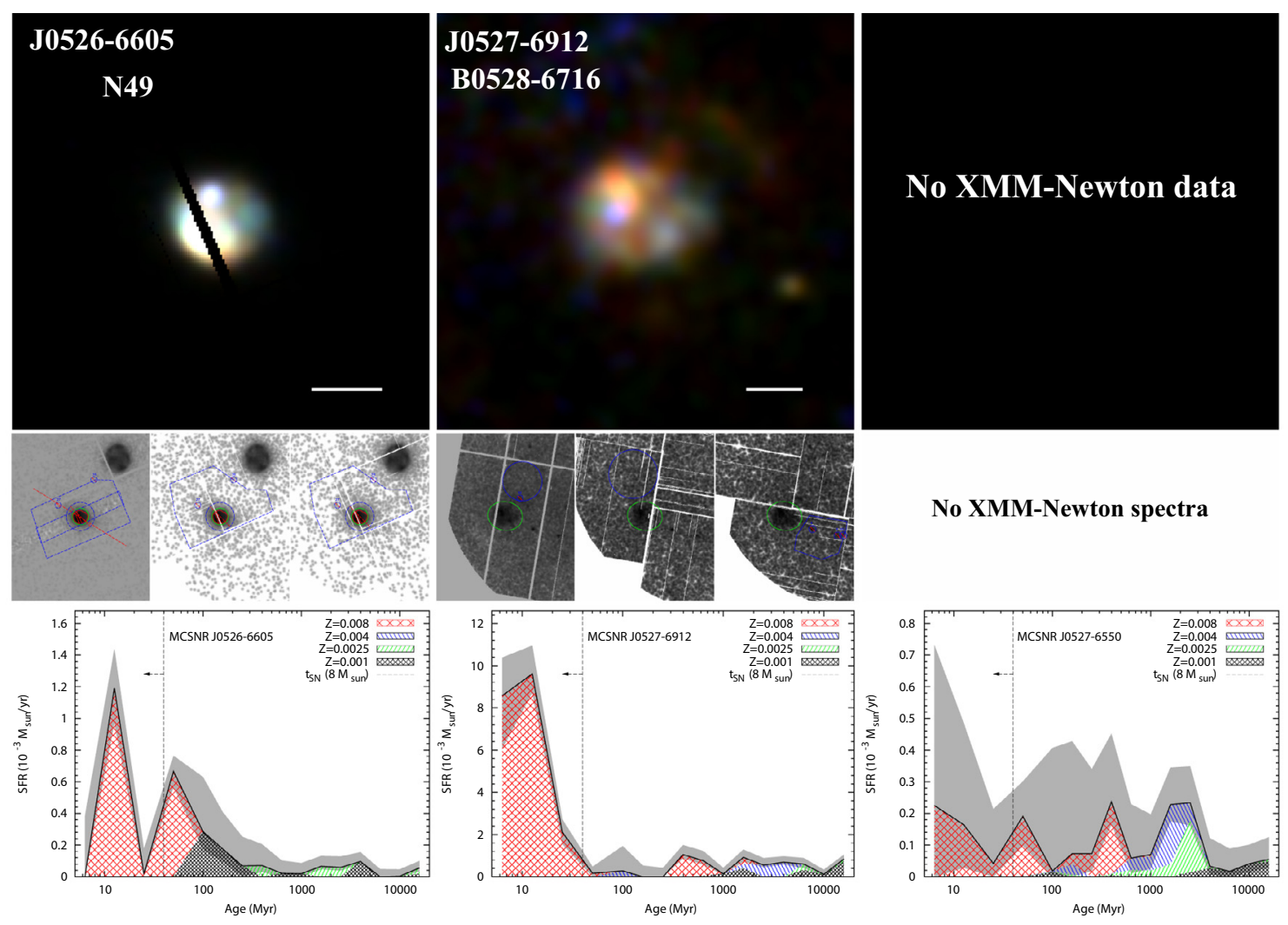

\section{No XMM-Newton spectra}
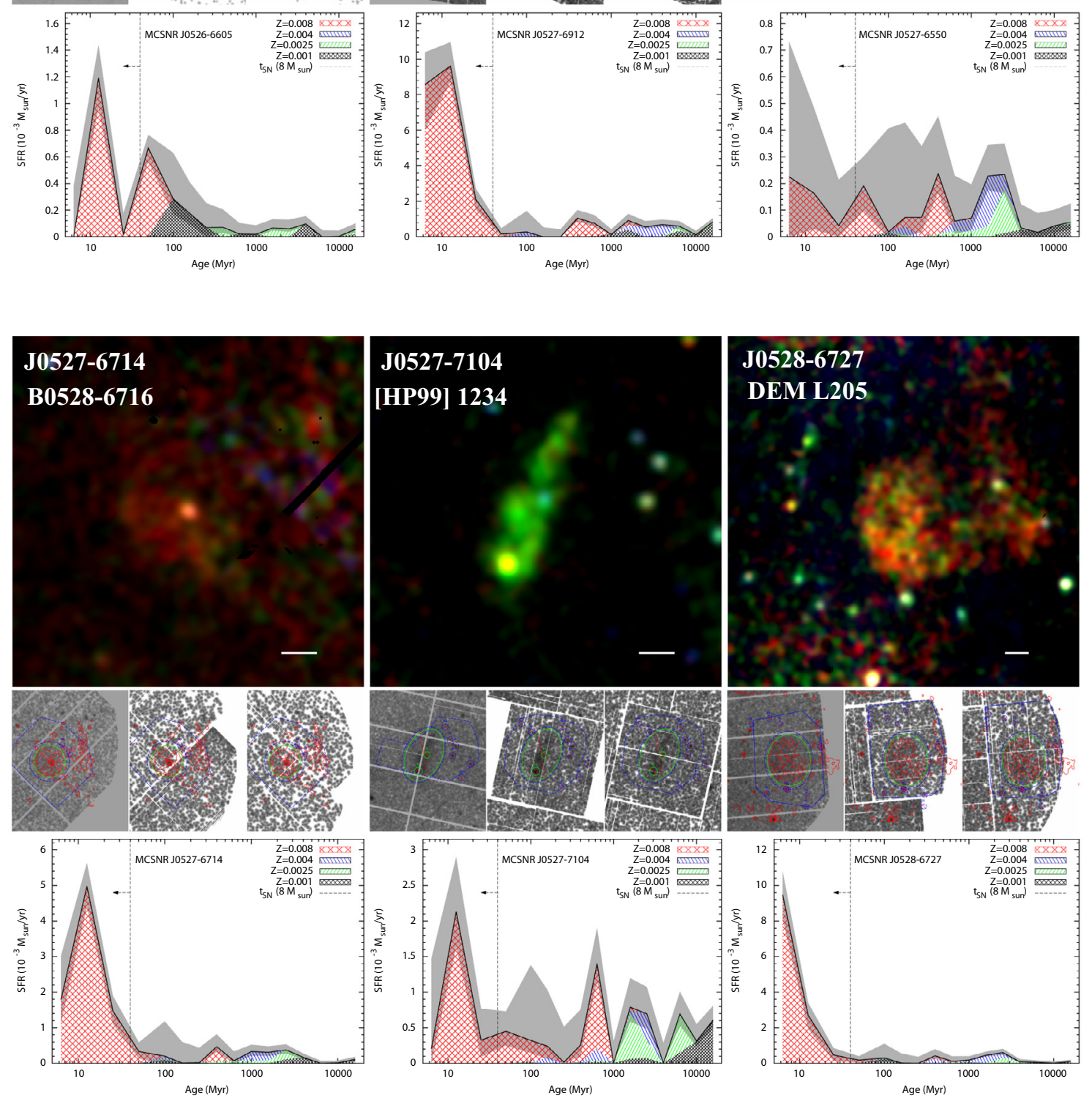
P. Maggi et al.: The population of X-ray supernova remnants in the Large Magellanic Cloud
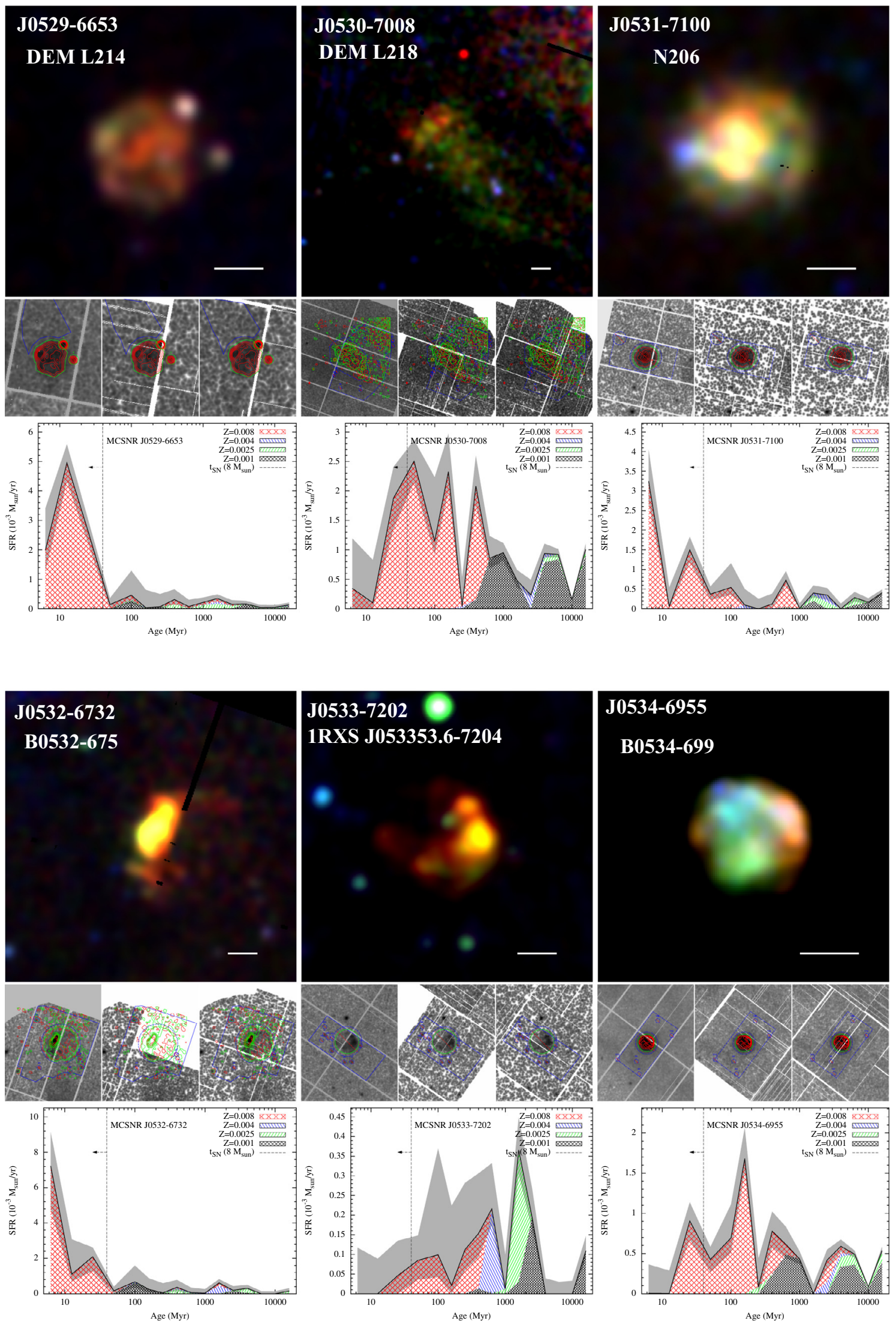


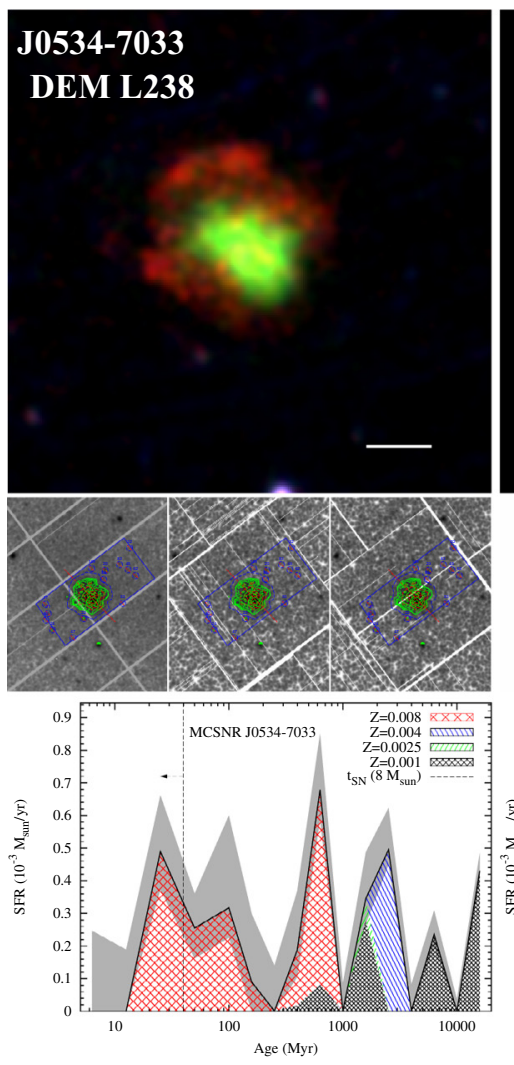

\section{J0535-6916 SNR 1987A}

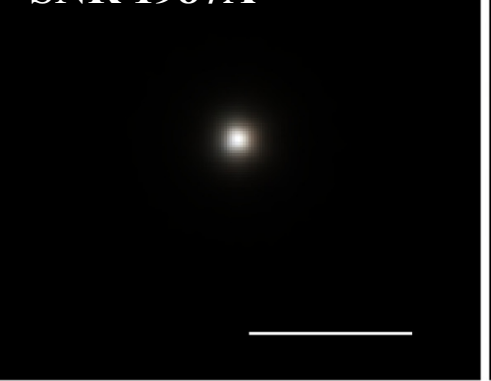

J0535-6602

N63A

See Sect. 5.3
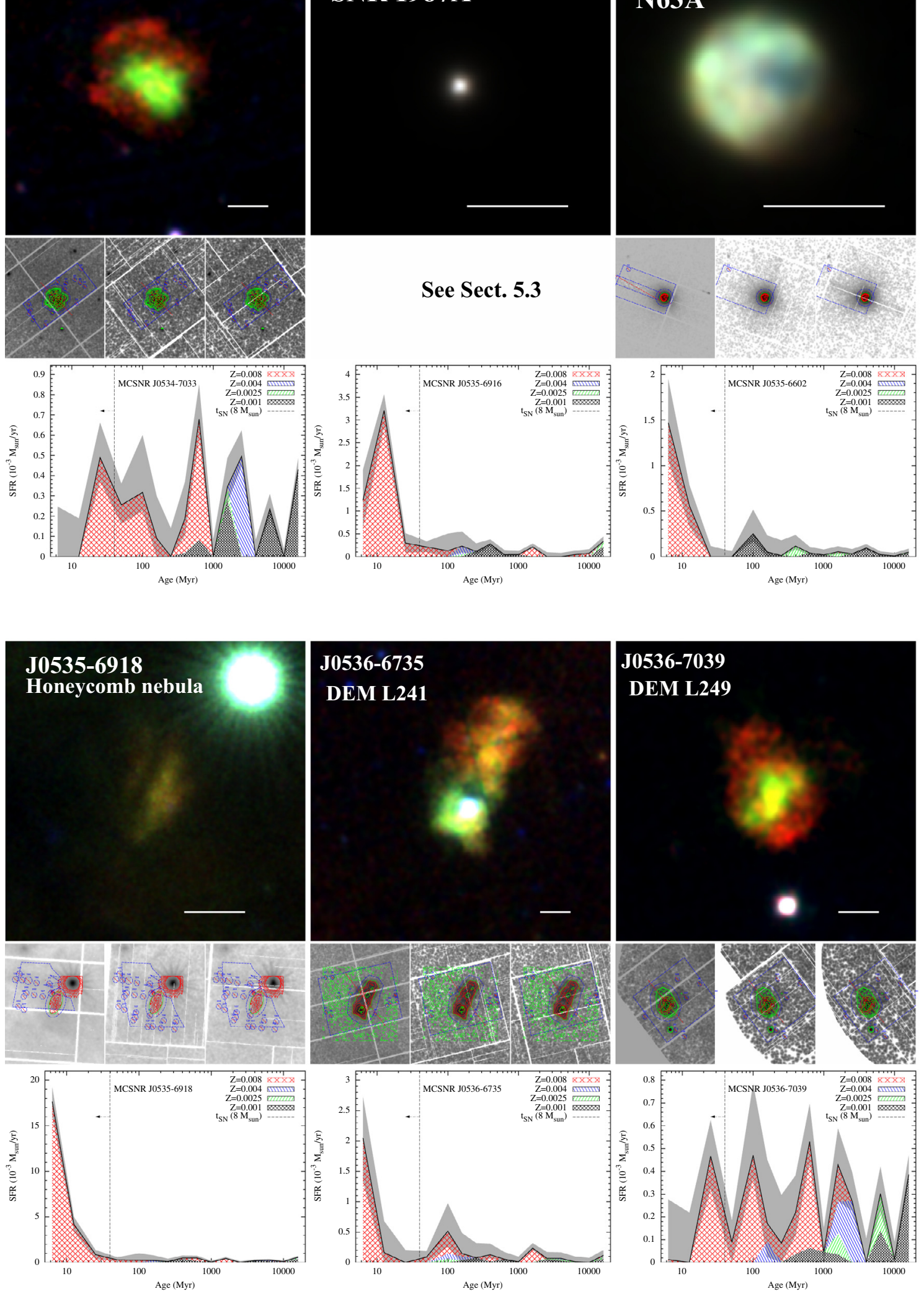
P. Maggi et al.: The population of X-ray supernova remnants in the Large Magellanic Cloud
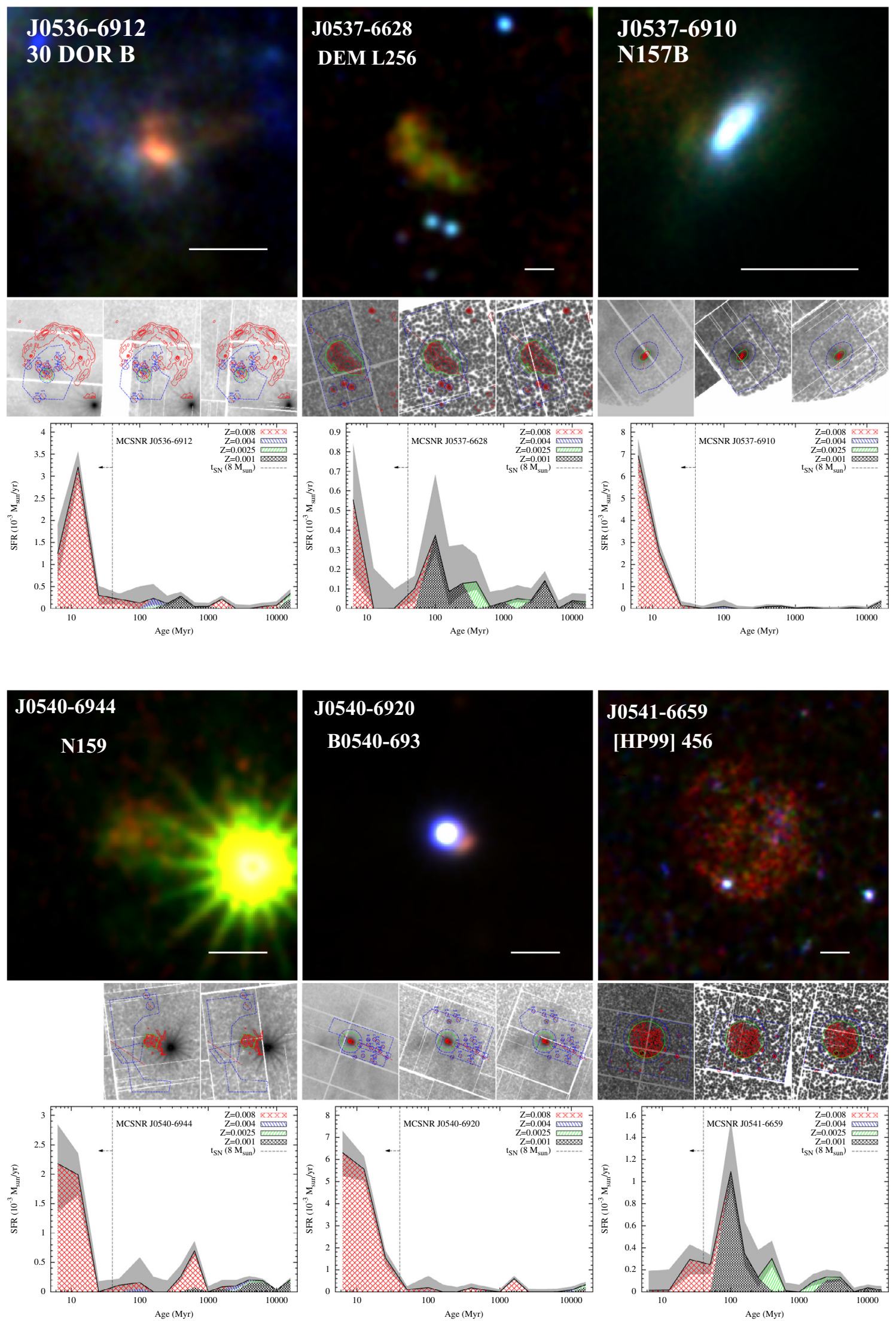

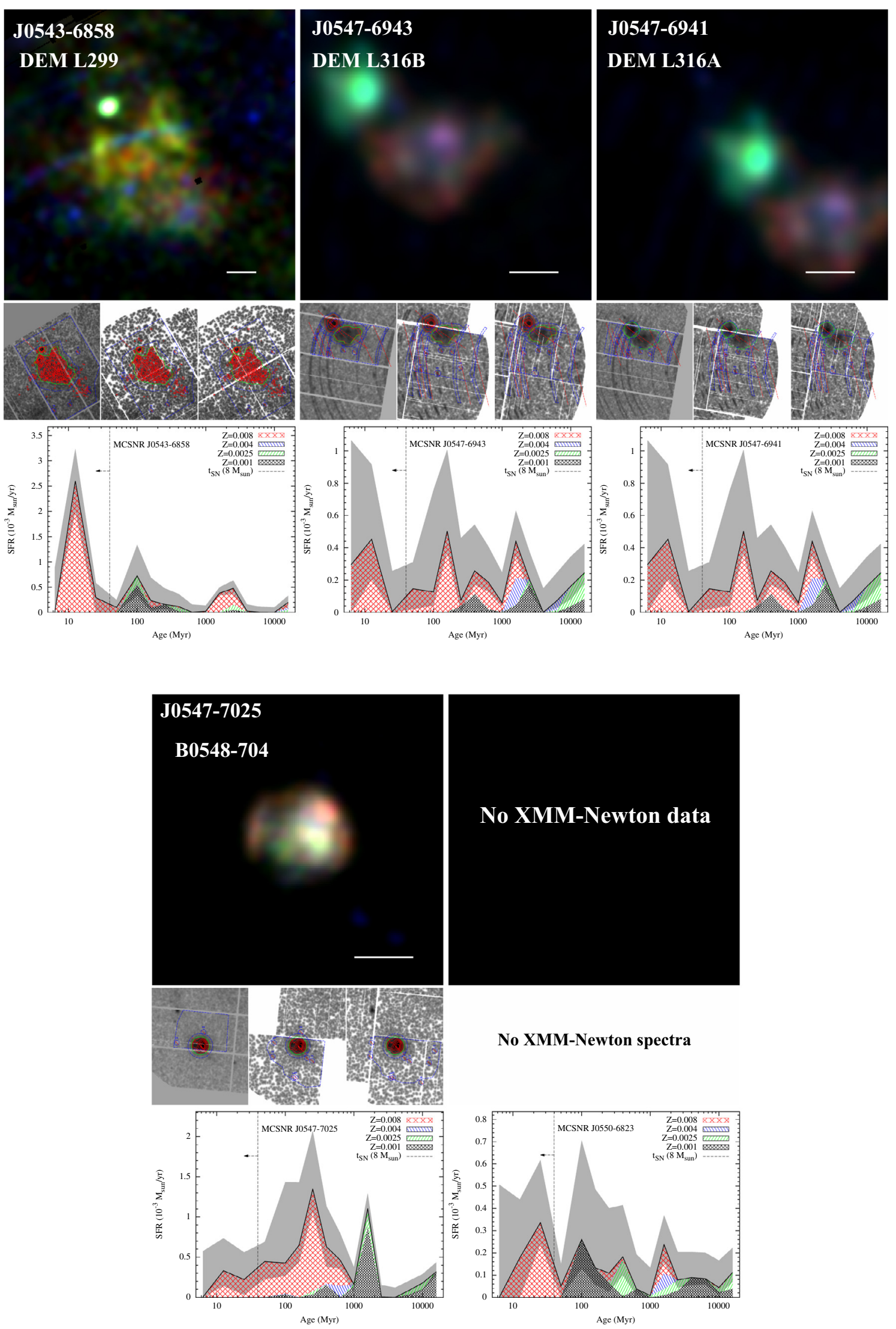

No XMM-Newton spectra

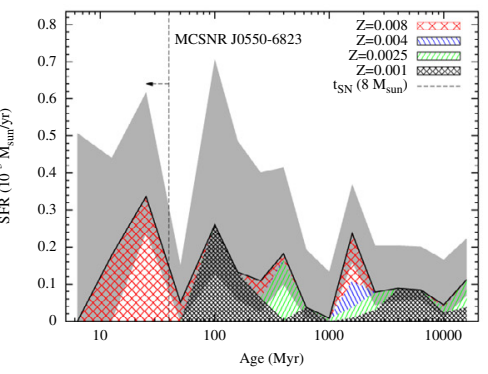

